\title{
A generalization of intrinsic geometry and an affine connection representation of gauge fields
}

\author{
Zhao-Hui Man* \\ 10-2-1102, Hua Yang Nian Hua Bei Qu, Yinchuan, Ningxia, China
}

\begin{abstract}
There are two ways to unify gravitational field and gauge field. One is to represent gravitational field as principal bundle connection, and the other is to represent gauge field as affine connection. Poincare gauge theory and metric-affine gauge theory adopt the first approach. This paper adopts the second.

We show a generalization of Riemannian geometry and a new affine connection, and apply them to establishing a unified coordinate description of gauge field and gravitational field. It has the following advantages.

(i) Gauge field and gravitational field have the same affine connection representation, and can be described by a unified spatial frame.

(ii) Time can be regarded as the total metric with respect to all dimensions of internal coordinate space and external coordinate space. On-shell can be regarded as gradient direction. Quantum theory can be regarded as a geometric theory of distribution of gradient directions. Hence, gravitational theory and quantum theory obtain the same view of time and space and a unified description of evolution in affine connection representation of gauge fields.

(iii) Chiral asymmetry, coupling constants, MNS mixing and CKM mixing can appear spontaneously in affine connection representation, while in $U(1) \times S U(2) \times S U(3)$ principal bundle connection representation they can just only be artificially set up. Some principles and postulates of the conventional theories that are based on principal bundle connection representation can be turned into theorems in affine connection representation, so they are not necessary to be regarded as principles or postulates anymore.
\end{abstract}

Keywords: affine connection representation of gauge fields, generalized intrinsic geometry, reference-system, simple connection, time metric, distribution of gradient directions

2010 MSC: 51P05, 70A05, 53Z05, 53C05, 58A05

\section{Contents}

1 Introduction $\quad 2$

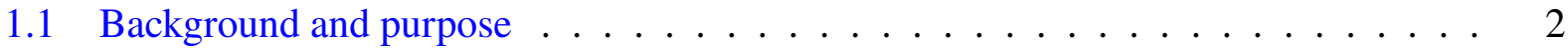

1.2 Ideas and methods . . . . . . . . . . . . . . . . . . 3

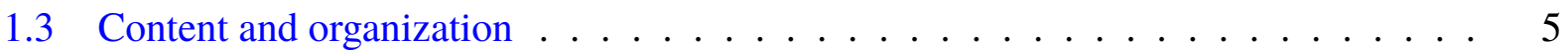

*Corresponding author. This article is registered under preprint number: arXiv:2004.02223 .

Email address: shetcslion@163.com (Zhao-Hui Man) 
2 Mathematical preparations $\quad 7$

2.1 Geometric manifold . . . . . . . . . . . . . . . . . . 7

2.2 Metric and semi-metric . . . . . . . . . . . . . . . . . 7

2.3 Gauge transformation in affine connection representation . . . . . . . . . . 8

2.4 Generalized intrinsic geometry . . . . . . . . . . . . . . . . 8

2.5 Simple connection ............................ 9

3 The evolution in affine connection representation of gauge fields 10

3.1 The relation between time and space . . . . . . . . . . . . . . . 10

3.2 Evolution path as a submanifold . . . . . . . . . . . . . . . 11

3.3 Evolution lemma . . . . . . . . . . . . . . . . . . 13

3.4 On-shell evolution as a gradient . . . . . . . . . . . . . . . . . 14

3.5 On-shell evolution of potential field and affine connection representation of YangMills equation . . . . . . . . . . . . . . . . . . 14

3.6 On-shell evolution of general charge and affine connection representation of mass, energy, momentum and action . . . . . . . . . . . . . . 16

3.7 Affine connection representation of interaction . . . . . . . . . . . . . 18

3.8 Inversion transformation in affine connection representation . . . . . . . . . . . 19

3.9 Two dual descriptions of gradient direction field . . . . . . . . . . . . . . 20

3.10 Quantum evolution as a distribution of gradient directions . . . . . . . . . . . 21

4 Affine connection representation of gauge fields in classical spacetime 26

4.1 Existence and uniqueness of classical spacetime submanifold . . . . . . . . . . 26

4.2 Gravitational field on classical spacetime submanifold . . . . . . . . . . . . . 28

4.3 Mathematical conversion between two coordinate representations . . . . . . . . . . . 30

4.4 Affine connection representation of classical spacetime evolution . . . . . . . . . 32

4.5 Affine connection representation of Dirac equation . . . . . . . . . . . 33

4.6 From classical spacetime back to full-dimensional space . . . . . . . . . . 36

5 Affine connection representation of the gauge field of weak-electromagnetic interaction 39

6 Affine connection representation of the gauge field of strong interaction 43

7 Affine connection representation of the unified gauge field 45

8 Conclusions $\quad 52$

\section{Introduction}

\subsection{Background and purpose}

We know that gravitational field can be described by the geometric theory on Riemannian manifold, and that gauge field can be described by the geometric theory on fibre bundle. There have been many theories trying to solve the problem that how to establish a unified description of gravitational field and gauge field.

The main difficulties of this problem in the above two geometric theories are as follows. 
(i) The affine connection that describes gravitational field is different from the abstract connection that describes gauge field. Gravitational field and gauge field are not described by the same connection.

(ii) The affine connection of gravitational field has an explicit sense of view of time and space, but the abstract connection of gauge field has not. Affine connection reflects the degrees of freedom of spacetime, but abstract connection just only reflects some abstract degrees of freedom.

(iii) Time is a component of gravitational field, but not a component of gauge field. Minkowski coordinates basically regard 1-dimensional time and 3-dimensional space on an equal footing. The description of evolution of gravitational field is essentially different from that of gauge field.

There are the following two ways to solve the problem (i).

One way is to represent gravitational field as principal bundle connection. We can take the transformation group $\operatorname{Gravi}(3,1)$ of gravitational field as the structure group of principal bundle to establish a gauge theory of gravitational field, the local transformation group of which is in the form of $\operatorname{Gravi}(3,1) \otimes_{s} \operatorname{Gauge}(n)$, e.g. Poincare gauge theory [1-11] and metric-affine gauge theory [12-23]. Gravitational field and gauge field can be uniformly described by connections on principal bundle. We point out that, this approach solves the problem (i) in form, but it cannot solve the problems (ii) and (iii).

The other way is to represent gauge field as affine connection. This is the approach adopted by this paper. In this way, gravitational field and gauge field can be uniformly described by affine connection, and both the problems (i) and (ii) can be solved. In addition, we find that in affine connection representation, time can be regarded as the total metric with respect to all dimensions of internal coordinate space and external coordinate space, so the problem (iii) can be solved.

The difficulty with the second approach is that, Riemannian manifold is not suitable for establishing an affine connection representation of gauge fields. In other words, an affine connection (e.g. Levi-Civita connection, spin connection, Riemmann-Cartan connection) that is dependent on Christoffel symbol $\left\{\rho_{\mu \nu}\right\}$ can describe gravitational field, but cannot describe general gauge field.

We propose some new mathematical tools to overcome the above difficulty, and establish a torsion-free affine connection representation of gauge fields in a geometry that is more general than Riemannian geometry. This is the main work of this paper.

In the theory of this paper, gravitational field and gauge field are unified in affine connection representation. Thus, the problems (i), (ii) and (iii) can all be solved. In addition, there is another advantage that some principles and postulates of the conventional theories that are based on principal bundle connection representation can be turned into theorems in affine connection representation, so they are not necessary to be regarded as principles or postulates anymore.

\subsection{Ideas and methods}

We divide the problem of establishing affine connection representation of gauge fields into three parts as follows.

(I) Which affine connection is suitable for describing not only gravitational field, but also gauge field?

(II) How to describe the evolution of gauge field and gravitational field in affine connection representation?

(III) What are the concrete forms of electromagnetic, weak and strong interaction fields in affine connection representation? 
For the problem (I). We need a new geometry in a new way to integrate Riemannian geometry and gauge field geometry. The new geometry is required to be more general than Riemannian geometry, and more concrete than the existing gauge field geometry.

It has long been known that the coefficients $G_{M N}=\delta_{A B} B_{M}^{A} B_{N}^{B}$ of metric tensor remain unchanged when orthogonal transformations act on the semi-metric $B_{M}^{A}$ (or to say frame field) [24, 25]. So we can say a Riemannian geometric property is an invariant under identical transformations of metric, and we can also speak of it as an invariant under orthogonal transformations of semimetric.

If we take different orthogonal transformations of semi-metric at different points of a Riemannian manifold, the Riemannian manifold remains unchanged. It is evident that local orthogonal transformations mean more general geometric properties. However, Riemannian manifold cannot reflect these geometric properties.

We see that the semi-metric $B_{M}^{A}$ is more fundamental than the metric $G_{M N}$. Therefore, we need to generalize the Riemannian manifold $\left(M, G_{M N}\right)$ to a more general manifold $\left(M, B_{M}^{A}\right)$, and then to regard orthogonal transformation of semi-metric as gauge transformation.

Next, we need an affine connection to describe gauge field. Riemann-Cartan geometry is a generalization of Riemannian geometry, its affine connection is independent of metric, but RiemannCartan connection and spin connection are both dependent on Christoffel symbol, so they are not suitable for describing gauge field.

We make a further generalization, and adopt a new torsion-free affine connection that is totally independent of Christoffel symbol, and use semi-metric to construct an affine connection representation of gauge fields. In this way, gravitational field and gauge field can be unified on a manifold $\left(M, B_{M}^{A}\right)$ that is defined by semi-metric.

Intrinsic geometry should not be confined to the point of view of metric and Christoffel symbol. Fig.1 is an example of 2-dimensional manifold $\left(M, B_{M}^{A}\right)$. It shows that semi-metric is indeed possessed of an intrinsic geometric significance independent of metric, and that it reflects the degree of slackness and tightness of the distribution of a coordinate frame in another coordinate frame.
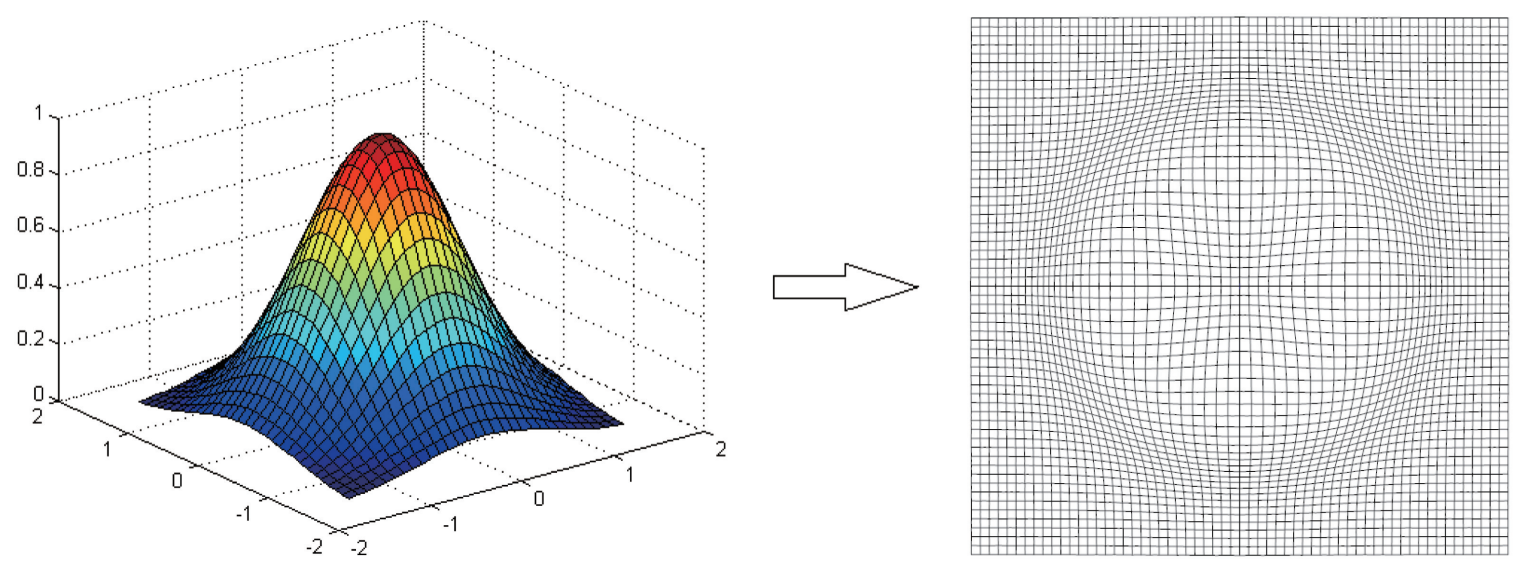

Figure 1: The intuition of intrinsic geometry of a surface

On a manifold $\left(M, B_{M}^{A}\right)$, we are able to use the semi-metric $B_{M}^{A}$ to endow particle fields and gauge fields with intrinsic geometric constructions.

In the theories based on principal bundle connection representation: 
(1) For particle fields. Several complex-valued functions which satisfy the Dirac equation, are sometimes used to refer to a charged lepton field $l$, and sometimes a neutrino field $\nu$. It is not clear that how to distinguish these field functions $l$ and $\nu$ by inherent mathematical constructions.

(2) For gauge fields. (i) In the electrodynamics, the electromagnetic potentials $A$ and $\varphi$ are abstract, they have no inherent mathematical constructions. In other words, the Levi-Civita connection $\Gamma_{\nu \rho}^{\mu}$ is constructed by the metric $g_{\mu \nu}$, but it is not clear that what geometric quantity $A$ and $\varphi$ are constructed by. (ii) In the QFT based on the Yang-Mills theory[26], such as Glashow-Weinberg-Salam's unified theory of weak and electromagnetic interactions [27-35], quantum chromodynamics[36-46] and various GUTs [47-52], as well as gravitational gauge GUTs [53-59], those connections which describe gauge fields are similar to the above $A$ and $\varphi$, and they are devoid of inherent mathematical constructions.

By contrast, in the affine connection representation of this paper, various gauge fields including $A$ and $\varphi$ and various particle fields including $l$ and $\nu$ are all constructed by semi-metric of internal coordinate space. Thus, they are not only abstract algebraic representations of group, but also possessed of concrete geometric entities.

For the problem (II). There is a fundamental difficulty that time is a component of gravitational field, but not a component of gauge field. This leads to an essential difference between the description of evolution of gravitational field and that of gauge field. In this case, it is difficult to obtain a unified theory of evolution in affine connection representation. We find that, we can define time as the total metric with respect to all dimensions of internal coordinate space and external coordinate space, and define evolution as one-parameter group of diffeomorphism, to overcome the above difficulty.

Now that gauge field and gravitational field are both represented as affine connection, then the properties that are related to gauge field, such as charge, current, mass, energy, momentum and action, must have corresponding affine connection representations. Thus, Yang-Mills equation, energy-momentum equation and Dirac equation are turned into geometric properties in gradient direction, in other words, on-shell evolution is characterized by gradient direction. Correspondingly, quantum theory can be interpreted as a geometric theory of distribution of gradient directions.

For the problem (III). Suppose $\Gamma_{N P}^{M}$ is the required affine connection, which is constructed by semi-metric $B_{M}^{A}$. The basic idea is that the components of $\Gamma_{N P}^{M}$ with $M, N \in\{4,5, \cdots, \mathfrak{D}\}$ describe electromagnetic, weak and strong interaction fields. The other components of $\Gamma_{N P}^{M}$ describe gravitational field.

\subsection{Content and organization}

In this paper, we are going to show how to construct the affine connection representation of gauge fields. Sections are organized as follows.

Corresponding to the problem (I), in section 2 we make some necessary mathematical preparations, and construct a new torsion-free affine connection that is different from Levi-Civita connection, spin connection and Riemann-Cartan connection. Meanwhile, we generalize the notion of reference system, and give it a strict mathematical definition. The reference system in conventional sense is just only defined on a local coordinate neighborhood, and it has only $(1+3)$ dimensions. But in this paper we define the concept of reference-system over the entire manifold. It is possessed of more dimensions but diffenrent from Kaluza-Klein theory [60-62] and string theories [63-75]. Thus, both of gravitational field and gauge field are regarded as special cases of such a concept of reference-system. 
Corresponding to the problem (II), in section 3 we establish the general theory of evolution in affine connection representation of gauge fields, and in section 4 we discuss the application of this general theory of evolution to $(1+3)$-dimensional classical spacetime.

Corresponding to the problem (III), in sections 5 to 7 we show concrete forms of affine connection representations of electromagnetic, weak and strong interaction fields.

Some topics are organized as follows.

(1) Time is regarded as the total metric with respect to all spatial dimensions including external coordinate space and internal coordinate space, see Definition 3.1.1 and Discussion 4.3.2 for detail. The CPT inversion is interpreted as the composition of the total inversion of coordinates and the total inversion of metrics, see section 3.8 for detail. The conventional $(1+3)$-dimensional Minkowski coordinate $x^{\mu}$ originates from the general $\mathfrak{D}$-dimensional coordinate $x^{M}$. The construction method of extra dimensions is different from those of Kaluza-Klein theory and string theory, see section 4.2 for detail.

(2) On-shell evolution is characterized by gradient direction field. See sections 3.4, 3.5, 3.6 and 4.4 for detail.

(3) Quantum theory is regarded as a geometric theory of distribution of gradient directions. We show two dual descriptions of gradient direction. They just exactly correspond to the Schrödinger picture and the Heisenberg picture. In these points of view, the gravitational theory and quantum theory become coordinated. They have a unified description of evolution, and the definition of Feynman propagator is simplified to a stricter form. See sections 3.9 and 3.10 for detail.

(4) Yang-Mills equation originates from a geometric property of gradient direction. We show the affine connection representation of Yang-Mills equation. See section 3.5 and section 4.6 for detail.

(5) Energy-momentum equation originates from a geometric property of gradient direction. We show the affine connection representation of mass, energy, momentum and action, see section 3.6, Definition 4.4.1 and Discussion 4.4.1 for detail. Furthermore, we also show the affine connection representation of Dirac equation, see section 4.5 for detail.

(6) Gauge field and particle field are two geometric fields both constructed by semi-metric. This paper is not going to adopt the way of taking several abstract functions $\nu, l, A_{\mu}^{K}$ and speaking of them directly as a neutrino field, an electric charged lepton field and a gauge field, but to show the inherent and geometric constructions of $\nu, l, A_{\mu}^{K}$ concretely. See Discussion 3.5.??, sections 5, 6 and Definition 7.2 for detail.

(7) The coupling constants of interactions between gauge field and particle field originate from the metric of the internal coordinate space of manifold. See sections 5, 6 and 7 for detail.

(8) Why do not neutrinos participate in the electromagnetic interactions? And why do not right-handed neutrinos participate in the weak interactions with $W$ bosons? In the Standard Model, they are both postulates. However, in the new framework, they are natural and geometric results of affine connection representation of gauge fields, therefore not necessary to be regarded as postulates anymore. See Proposition 5.2 and Proposition 7.1 for detail.

(9) Why do leptons and quarks both have three generations? We give an answer in section 7. It is a natural geometric result of affine connection representation of gauge fields. 


\section{Mathematical preparations}

\subsection{Geometric manifold}

In order to establish the affine connection representation of gauge fields, we have to generalize Riemannian manifold to geometric manifold.

Definition 2.1.1. Let $M$ be a $\mathfrak{D}$-dimensional connected smooth real manifold. $\forall p \in M$, take a coordinate chart $\left(U_{p}, \varphi_{U p}\right)$ on a neighborhood $U_{p}$ of $p$. They constitute a coordinate covering

$$
\varphi \triangleq\left\{\left(U_{p}, \varphi_{U p}\right)\right\}_{p \in M}
$$

which is said to be a point-by-point covering. For the sake of simplicity, $U_{p}$ can be denoted by $U$, and $\varphi_{U p}$ by $\varphi_{U}$.

Let $\varphi$ and $\psi$ be two point-by-point coverings. For the two coordinate frames $\varphi_{U}$ and $\psi_{U}$ on the neighborhood $U$ of point $p$, if

$$
f_{p} \triangleq \varphi_{U} \circ \psi_{U}^{-1}: \psi_{U}(U) \rightarrow \varphi_{U}(U), \quad \xi^{A} \mapsto x^{M}
$$

is a smooth homeomorphism, $f_{p}$ is called a local reference-system, where $\psi_{U}$ is said to be the basis coordinate frame of $f_{p}$, and $\varphi_{U}$ is said to be the performance coordinate frame of $f_{p}$.

If every $p \in M$ is endowed with a local reference-system $f(p)$, and we require the semi-metrics $B_{M}^{A}$ and $C_{A}^{M}$ in section 2.2 to be smooth real functions on $M$, then

$$
f: M \rightarrow R E F, \quad p \mapsto f(p)
$$

is said to be a reference-system on $M$, and $(M, f)$ is said to be a geometric manifold.

\subsection{Metric and semi-metric}

In the absence of a special declaration, the indices take values as $A, B, C, D, E=1,2, \cdots, \mathfrak{D}$ and $M, N, P, Q, R=1,2, \cdots, \mathfrak{D}$. The derivative functions

$$
b_{M}^{A} \triangleq \frac{\partial \xi^{A}}{\partial x^{M}}, \quad c_{A}^{M} \triangleq \frac{\partial x^{M}}{\partial \xi^{A}}
$$

of $f(p)$ on $U_{p}$ define the semi-metrics (or to say frame field) $B_{M}^{A}$ and $C_{A}^{M}$ of $f$ on the manifold $M$, that are

$$
B_{M}^{A}: M \rightarrow \mathbb{R}, p \mapsto B_{M}^{A}(p) \triangleq\left(b_{f(p)}\right)_{M}^{A}(p), \quad C_{A}^{M}: M \rightarrow \mathbb{R}, p \mapsto C_{A}^{M}(p) \triangleq\left(c_{f(p)}\right)_{A}^{M}(p)
$$

Let $\delta_{A B}=\delta^{A B}=\delta_{B}^{A}=\operatorname{Kronecker}(A, B)$ and $\varepsilon_{M N}=\varepsilon^{M N}=\varepsilon_{N}^{M}=\operatorname{Kronecker}(M, N)$. The metric tensors of $f$ are

$$
G_{M N}=\delta_{A B} B_{M}^{A} B_{N}^{B}, \quad H_{A B}=\varepsilon_{M N} C_{A}^{M} C_{B}^{N} .
$$

Similarly, it can also be defined that $\bar{b}_{A}^{M} \triangleq \frac{\partial \xi_{A}}{\partial x_{M}}, \bar{c}_{M}^{A} \triangleq \frac{\partial x_{M}}{\partial \xi_{A}}$ and corresponding $\bar{B}_{A}^{M}, \quad \bar{C}_{M}^{A}$. 


\subsection{Gauge transformation in affine connection representation}

$\forall p \in M, f(p) \triangleq \rho_{U} \circ \psi_{U}^{-1}$ induces local reference-system transformations

$$
\begin{aligned}
& L_{f(p)}: k(p) \triangleq \psi_{U} \circ \varphi_{U}^{-1} \mapsto \rho_{U} \circ \varphi_{U}^{-1}=f(p) \circ k(p), \\
& R_{f(p)}: h(p) \triangleq \varphi_{U} \circ \rho_{U}^{-1} \mapsto \varphi_{U} \circ \psi_{U}^{-1}=h(p) \circ f(p),
\end{aligned}
$$

and reference-system transformations on manifold $M$

$$
L_{f}: p \mapsto L_{f(p)}, \quad R_{f}: p \mapsto R_{f(p)} .
$$

We also speak of $L_{f}$ and $R_{f}$ as (affine) gauge transformations, see Discussion 4.6 .3 for reasons.

(i) If $\left[B_{M}^{A}\right]$ of $f$ is the identity matrix, then $L_{f}$ and $R_{f}$ are called identical transformations.

(ii) If $\forall p_{1}, p_{2} \in M, B_{M}^{A}\left(p_{1}\right)=B_{M}^{A}\left(p_{2}\right)$, then $L_{f}$ and $R_{f}$ are called flat transformations.

(iii) If $\delta_{A B} B_{M}^{A} B_{N}^{B}=\varepsilon_{M N}$, then $L_{f}$ and $R_{f}$ are called orthogonal transformations.

The totality of all reference-system transformations on $M$ is denoted by $G L(M)$, which is a subgroup of $\bigotimes_{p \in M} G L(\mathfrak{D}, \mathbb{R})_{p}$, where $\bigotimes$ represents external direct product.

\subsection{Generalized intrinsic geometry}

Let the notation $F_{k}$ represent $L_{k}$ or $R_{k}$. The above transforamtions lead to the following relations of equivalence.

(i) Define a relation of equivalence $\cong$ such that $f \cong g$ and $(M, f) \cong(M, g)$ if and only if $\forall p \in M,\left(B_{f}\right)_{M}^{A}(p)=\left(B_{g}\right)_{M}^{A}(p)$. The corresponding equivalence classes are denoted by $[f]$ and $(M,[f])$.

(ii) Define a relation of equivalence $\simeq$ such that $f \simeq g$ and $(M, f) \simeq(M, g)$ if and only if there exists a flat transformation $F_{k}$ such that $F_{k}(f)=g$. The corresponding equivalence classes are denoted by $|f|$ and $(M,|f|)$.

(iii) Define a relation of equivalence $\simeq_{O}$ such that $f \simeq_{O} g$ and $(M, f) \simeq_{O}(M, g)$ if and only if there exists an orthogonal transformation $F_{k}$ such that $F_{k}(f)=g$. The corresponding equivalence classes are denoted by $[f]_{O}$ and $\left(M,[f]_{O}\right)$.

(iv) Define a relation of equivalence $\sim$ such that $f \sim g$ and $(M, f) \sim(M, g)$ if and only if there exists an arbitrary transformation $F_{k}$ such that $F_{k}(f)=g$. The corresponding equivalence classes are denoted by $[f]_{U}$ and $\left(M,[f]_{U}\right)$.

The totality of all the geometric manifolds on $M$ is denoted by $\mathcal{M}(M)$. The above four relations of equivalence determine the following four classifications.

$$
\mathcal{M}(M) / \cong, \quad \mathcal{M}(M) / \simeq, \quad \mathcal{M}(M) / \simeq_{O}, \quad \mathcal{M}(M) / \sim .
$$

The largest one is $\mathcal{M}(M) / \cong$, which is said to be (generalized) intrinsic geometry. The smallest one is $\mathcal{M}(M) / \sim$, which is said to be universal geometry.

Let the notation $h X$ represent a geometric property of $X$. We have the following conclusions.

(i) $\forall(M,[f]) \in \mathcal{M}(M) / \cong, h(M,[f])$ is an invariant under identical transformation.

(ii) $\forall(M,|f|) \in \mathcal{M}(M) / \simeq, h(M,|f|)$ is an invariant under flat transformation.

(iii) $\forall\left(M,[f]_{O}\right) \in \mathcal{M}(M) / \simeq_{O}, h\left(M,[f]_{O}\right)$ is an invariant under orthogonal transformation.

(iv) $\forall\left(M,[f]_{U}\right) \in \mathcal{M}(M) / \sim, \quad h\left(M,[f]_{U}\right)$ is an invariant under arbitrary transformation. 
If detailed physical properties are described by geometric properties on $\mathcal{M}(M) / \cong$, then universal physical properties can necessarily be described by geometric properties on $\mathcal{M}(M) / \sim$. Therefore, general covariance and gauge invariance are both geometric properties on $\mathcal{M}(M) / \sim$, they remain unchanged under arbitrary transformations of reference-system.

It is evident that $f \simeq_{O} g$ if and only if $\left(G_{f}\right)_{M N}=\left(G_{g}\right)_{M N}$. So $\mathcal{M}(M) / \simeq_{O}$ is Riemannian geometry, and $\left(M,[f]_{O}\right)$ is just exactly Riemannian manifold.

Due to $G_{M N}=\delta_{A B} B_{M}^{A} B_{N}^{B}$ we know that $\left(B_{f}\right)_{M}^{A}=\left(B_{g}\right)_{M}^{A}$ ensures $\left(G_{f}\right)_{M N}=\left(G_{g}\right)_{M N}$, but conversely when $\left(G_{f}\right)_{M N}=\left(G_{g}\right)_{M N}$ we have $\left(B_{f}\right)_{M}^{A} \neq\left(B_{g}\right)_{M}^{A}$ in general. So $(M, f)$ and $(M, g)$ are the same Riemannian manifold, but different geometric manifolds.

Under an orthogonal affine gauge transformation $L_{k}$, the metric $G_{M N}$ and the Christoffel symbol $\left\{\begin{array}{l}M \\ N P\end{array}\right\}$ both remain unchanged, therefore affine connection representation of gauge fields cannot be constructed with $G_{M N}$ and $\left\{\begin{array}{l}M \\ N P\end{array}\right\}$ on Riemannian geometry $\mathcal{M}(M) / \simeq_{O}$, but can only be constructed with $B_{M}^{A}$ and $C_{A}^{M}$ on generalized intrinsic geometry $\mathcal{M}(M) / \cong$.

\subsection{Simple connection}

We do not take into account spin connection and Riemann-Cartan connection, because they are dependent on Christoffel symbol $\left\{\begin{array}{l}M \\ N P\end{array}\right\}$. A nice choice is to adopt the following definition, which is independent of Christoffel symbol.

Definition 2.5.1. Let there be an affine connection $D$. It presents as

$$
D \frac{\partial}{\partial x^{N}} \triangleq \Gamma_{N P}^{M} d x^{P} \otimes \frac{\partial}{\partial x^{M}}, \quad D d x^{N} \triangleq-\Gamma_{M P}^{N} d x^{P} \otimes d x^{M} .
$$

If the affine connection coefficients are defined as

$$
\Gamma_{N P}^{M} \triangleq \frac{1}{2} C_{A}^{M}\left(\frac{\partial B_{N}^{A}}{\partial x^{P}}+\frac{\partial B_{P}^{A}}{\partial x^{N}}\right)=-\frac{1}{2}\left(B_{N}^{A} \frac{\partial C_{A}^{M}}{\partial x^{P}}+B_{P}^{A} \frac{\partial C_{A}^{M}}{\partial x^{N}}\right),
$$

$D$ is said to be a simple connection. In addition, denote $\Gamma_{M N P} \triangleq G_{M M^{\prime}} \Gamma_{N P}^{M^{\prime}}$. It is easy to verify that

$$
\Gamma_{M N P}=\frac{1}{2} \delta_{A B} B_{M}^{B}\left(\frac{\partial B_{N}^{A}}{\partial x^{P}}+\frac{\partial B_{P}^{A}}{\partial x^{N}}\right),
$$

and that $D$ is torsion-free. If we do not require it to be torsion-free, it is fine to adopt $\Gamma_{N P}^{M} \triangleq C_{A}^{M} \frac{\partial B_{N}^{A}}{\partial x^{P}}$.

Proposition 2.5.1. Suppose there are reference-systems $g$ and $k$ on a manifold $M$. Let the semimetrics of $g$ be

$$
\left(B_{g}\right)_{M}^{A}, \quad\left(C_{g}\right)_{A}^{M}
$$

Let the semi-metrics of $k$ be

$$
\left(B_{k}\right)_{M^{\prime}}^{M}, \quad\left(C_{k}\right)_{M}^{M^{\prime}}
$$

And let the semi-metrics of $g^{\prime} \triangleq L_{k}(g)$ be

$$
\left(B_{g^{\prime}}\right)_{M^{\prime}}^{A}=\left(B_{g}\right)_{M}^{A}\left(B_{k}\right)_{M^{\prime}}^{M}, \quad\left(C_{g^{\prime}}\right)_{A}^{M^{\prime}}=\left(C_{g}\right)_{A}^{M}\left(C_{k}\right)_{M}^{M^{\prime}}
$$

Then let the simple connections of $(M, g),(M, k),\left(M, g^{\prime}\right)$ be $\left(\Gamma_{g}\right)_{N P}^{M},\left(\Gamma_{k}\right)_{N^{\prime} P^{\prime}}^{M^{\prime}},\left(\Gamma_{g^{\prime}}\right)_{N^{\prime} P^{\prime}}^{M^{\prime}}$, respectively. Thus,

$$
\left(\Gamma_{g^{\prime}}\right)_{N^{\prime} P^{\prime}}^{M^{\prime}}=\left(\Gamma_{g}\right)_{N P}^{M}\left(C_{k}\right)_{M}^{M^{\prime}}\left(B_{k}\right)_{N^{\prime}}^{N}\left(B_{k}\right)_{P^{\prime}}^{P}+\left(\Gamma_{k}\right)_{N^{\prime} P^{\prime}}^{M^{\prime}}
$$


Proof. We just need to calculate it.

$$
\begin{aligned}
& \left(\Gamma_{g^{\prime}}\right)_{N^{\prime} P^{\prime}}^{M^{\prime}} \triangleq \frac{1}{2}\left(C_{g^{\prime}}\right)_{A}^{M^{\prime}}\left(\frac{\partial\left(B_{g^{\prime}}\right)_{N^{\prime}}^{A}}{\partial x^{P^{\prime}}}+\frac{\partial\left(B_{g^{\prime}}\right)_{P^{\prime}}^{A}}{\partial x^{N^{\prime}}}\right) \\
= & \frac{1}{2}\left(C_{k}\right)_{M}^{M^{\prime}}\left(C_{g}\right)_{A}^{M}\left(\frac{\partial\left(\left(B_{k}\right)_{N^{\prime}}^{N}\left(B_{g}\right)_{N}^{A}\right)}{\partial x^{P^{\prime}}}+\frac{\partial\left(\left(B_{k}\right)_{P^{\prime}}^{P}\left(B_{g}\right)_{P}^{A}\right)}{\partial x^{N^{\prime}}}\right) \\
= & \frac{1}{2}\left(C_{k}\right)_{M}^{M^{\prime}}\left(C_{g}\right)_{A}^{M}\left(\frac{\partial\left(B_{k}\right)_{N^{\prime}}^{N}}{\partial x^{P^{\prime}}}\left(B_{g}\right)_{N}^{A}+\left(B_{k}\right)_{N^{\prime}}^{N} \frac{\partial\left(B_{g}\right)_{N}^{A}}{\partial x^{P^{\prime}}}+\frac{\partial\left(B_{k}\right)_{P^{\prime}}^{P}}{\partial x^{N^{\prime}}}\left(B_{g}\right)_{P}^{A}+\left(B_{k}\right)_{P^{\prime}}^{P} \frac{\partial\left(B_{g}\right)_{P}^{A}}{\partial x^{N^{\prime}}}\right) \\
= & \frac{1}{2}\left(C_{k}\right)_{M}^{M^{\prime}}\left(C_{g}\right)_{A}^{M}\left(\left(B_{k}\right)_{N^{\prime}}^{N} \frac{\partial\left(B_{g}\right)_{N}^{A}}{\partial x^{P^{\prime}}}+\left(B_{k}\right)_{P^{\prime}}^{P} \frac{\partial\left(B_{g}\right)_{P}^{A}}{\partial x^{N^{\prime}}}\right) \\
+ & \frac{1}{2}\left(C_{k}\right)_{M}^{M^{\prime}}\left(C_{g}\right)_{A}^{M}\left(\frac{\partial\left(B_{k}\right)_{N^{\prime}}^{N}}{\partial x^{P^{\prime}}}\left(B_{g}\right)_{N}^{A}+\frac{\partial\left(B_{k}\right)_{P^{\prime}}^{P}}{\partial x^{N^{\prime}}}\left(B_{g}\right)_{P}^{A}\right) \\
= & \frac{1}{2}\left(C_{k}\right)_{M}^{M^{\prime}}\left(C_{g}\right)_{A}^{M}\left(\frac{\partial\left(B_{g}\right)_{N}^{A}}{\partial x^{P}}+\frac{\partial\left(B_{g}\right)_{P}^{A}}{\partial x^{N}}\right)\left(B_{k}\right)_{N^{\prime}}^{N}\left(B_{k}\right)_{P^{\prime}}^{P}+\frac{1}{2}\left(C_{k}\right)_{M}^{M^{\prime}}\left(\frac{\partial\left(B_{k}\right)_{N^{\prime}}^{M}}{\partial x^{P^{\prime}}}+\frac{\partial\left(B_{k}\right)_{P^{\prime}}^{M}}{\partial x^{N^{\prime}}}\right) \\
= & \left(\Gamma_{g}\right)_{N P}^{M}\left(C_{k}\right)_{M}^{M^{\prime}}\left(B_{k}\right)_{N^{\prime}}^{N}\left(B_{k}\right)_{P^{\prime}}^{P}+\left(\Gamma_{k}\right)_{N^{\prime} P^{\prime}}^{M^{\prime}}
\end{aligned}
$$

Remark 2.5.1. The above proposition is applied to Discussion 4.6.3. It induces the affine connection representation of gauge transformation.

Remark 2.5.2. When $G_{M N}=\delta_{A B} B_{M}^{A} B_{N}^{B}=$ const, we have $B_{M}^{A} \neq$ const in general. So LeviCivita connection $\Lambda_{N P}^{M}=0$, and simple connection $\Gamma_{N P}^{M} \triangleq \frac{1}{2} C_{A}^{M}\left(\frac{\partial B_{N}^{A}}{\partial x^{P}}+\frac{\partial B_{P}^{A}}{\partial x^{N}}\right) \neq 0$. Then, the corresponding Riemannian curvatures

$$
\begin{aligned}
K_{N P Q}^{M} & \triangleq \frac{\partial \Lambda_{N Q}^{M}}{\partial x^{P}}-\frac{\partial \Lambda_{N P}^{M}}{\partial x^{Q}}+\Lambda_{N Q}^{H} \Lambda_{H P}^{M}-\Lambda_{N P}^{H} \Lambda_{H Q}^{M}=0, \\
R_{N P Q}^{M} & \triangleq \frac{\partial \Gamma_{N Q}^{M}}{\partial x^{P}}-\frac{\partial \Gamma_{N P}^{M}}{\partial x^{Q}}+\Gamma_{N Q}^{H} \Gamma_{H P}^{M}-\Gamma_{N P}^{H} \Gamma_{H Q}^{M} \neq 0 .
\end{aligned}
$$

This shows that simple connection reflects more curved-properties of a manifold than Levi-Civita connection.

\section{The evolution in affine connection representation of gauge fields}

Now that we have the required affine connection, next we have to solve the problem that how to describe the evolution in affine connection representation.

In the existing theories, time is a component of gravitational field, but not a component of gauge field. This leads to an essential difference between the description of evolution of gravitational field and that of gauge field. In this case, it is difficult to obtain a unified theory of evolution in affine connection representation. We adopt the following way to overcome this difficulty.

\subsection{The relation between time and space}

Definition 3.1.1. Suppose $M=P \times N$ and $r \triangleq \operatorname{dim} P=3$. Let

$$
A, B, M, N=1, \cdots, \mathfrak{D} ; \quad s, i=1, \cdots, r ; \quad a, m=r+1, \cdots, \mathfrak{D} .
$$


On the geometric manifold $(M, f)$, the $d \xi^{0}$ and $d x^{0}$ which are defined by

$$
\begin{aligned}
& \left(d \xi^{0}\right)^{2} \triangleq \sum_{A=1}^{\mathfrak{D}}\left(d \xi^{A}\right)^{2}=\delta_{A B} d \xi^{A} d \xi^{B}=G_{M N} d x^{M} d x^{N} \\
& \left(d x^{0}\right)^{2} \triangleq \sum_{M=1}^{\mathfrak{D}}\left(d x^{M}\right)^{2}=\varepsilon_{M N} d x^{M} d x^{N}=H_{A B} d \xi^{A} d \xi^{B}
\end{aligned}
$$

are said to be total space metrics or time metrics. We also suppose

$$
\begin{aligned}
\left(d \xi^{(P)}\right)^{2} \triangleq \sum_{s=1}^{r}\left(d \xi^{s}\right)^{2}, & \left(d \xi^{(N)}\right)^{2} \triangleq \sum_{a=r+1}^{\mathfrak{D}}\left(d \xi^{a}\right)^{2} \\
\left(d x^{(P)}\right)^{2} \triangleq \sum_{i=1}^{r}\left(d x^{i}\right)^{2}, & \left(d x^{(N)}\right)^{2} \triangleq \sum_{m=r+1}^{\mathfrak{D}}\left(d x^{m}\right)^{2} .
\end{aligned}
$$

$d \xi^{(N)}$ and $d x^{(N)}$ are regarded as proper-time metrics. For convenience, $P$ is said to be external space and $N$ is said to be internal space.

Remark 3.1.1. The above definition implies a new view of time and space. The relation between time and space in this way is different from the Minkowski coordinates $x^{\mu}(\mu=0,1,2,3)$. Time and space are not the components on an equal footing anymore, but have a relation of total to component. It can be seen later that time reflects the total evolution in the full-dimensional space, while a specific spatial dimension reflects just a partial evolution in a specific direction.

The relation of the above concept to Minkowski coordinates is discussed detailedly in section 4 .

\subsection{Evolution path as a submanifold}

Definition 3.2.1. Let there be two reference-systems $f$ and $g$ on a manifold $M$. If $\forall p \in M$, $f(p) \triangleq \phi_{U} \circ \psi_{U}^{-1}$ and $g(p) \triangleq \phi_{U} \circ \rho_{U}^{-1}$ have the same performance coordinate frame $\phi_{U}$, namely it can be intuitively expressed as a diagram

$$
\psi_{U}(U) \stackrel{f(p)}{\longrightarrow} \phi_{U}(U) \stackrel{g(p)}{\longleftarrow} \rho_{U}(U),
$$

we say $f$ and $g$ motion relatively and interact mutually, and also say that $f$ evolves in $g$, or $f$ evolves on the geometric manifold $(M, g)$. Meanwhile, $g$ evolves in $f$, or to say $g$ evolves on $(M, f)$.

Let there be a one-parameter group of diffeomorphisms

$$
\varphi_{X}: M \times \mathbb{R} \rightarrow M
$$

acting on $M$, such that $\varphi_{X}(p, 0)=p$. Thus, $\varphi_{X}$ determines a smooth tangent vector field $X$ on $M$. If $X$ is nonzero everywhere, we say $\varphi_{X}$ is a set of evolution paths, and $X$ is an evolution direction field. Let $T \subseteq \mathbb{R}$ be an interval, then the regular imbedding

$$
L_{p} \triangleq \varphi_{X, p}: T \rightarrow M, t \mapsto \varphi_{X}(p, t)
$$


is said to be an evolution path through $p$. The tangent vector $\frac{d}{d t} \triangleq\left[L_{p}\right]=X(p)$ is called an evolution direction at $p$. For the sake of simplicity, we also denote $L_{p} \triangleq L_{p}(T) \subset M$, then

$$
\pi: L_{p} \rightarrow M, q \mapsto q
$$

is also a regular imbedding. If it is not necessary to emphasize the point $p, L_{p}$ is denoted by $L$ concisely.

In order to describe physical evolution, next we are going to strictly describe the mathematical properties of the reference-systems $f$ and $g$ sending onto the evolution path $L$.

Definition 3.2.2. Let the coordinates of $\left(U, \psi_{U}\right),\left(U, \phi_{U}\right),\left(U, \rho_{U}\right)$ be $\xi^{A}, x^{M}, \zeta^{A}$, respectively, and the corresponding time metrics $d \xi^{0}, d x^{0}, d \zeta^{0}$. On $U_{L} \triangleq U \cap L_{p}$ we have parameter equations

$$
\begin{aligned}
& \xi^{A}=\xi^{A}\left(x^{0}\right), \quad x^{M}=x^{M}\left(\xi^{0}\right), \quad \zeta^{A}=\zeta^{A}\left(x^{0}\right), \\
& \xi^{0}=\xi^{0}\left(x^{0}\right), \quad x^{0}=x^{0}\left(\xi^{0}\right), \quad \zeta^{0}=\zeta^{0}\left(x^{0}\right) \text {. }
\end{aligned}
$$

Take $f$ for example, according to Eq.(12), on $U_{L}$ we define

$$
\begin{aligned}
& b_{0}^{A} \triangleq \frac{d \xi^{A}}{d x^{0}}, \quad b_{0}^{0} \triangleq \frac{d \xi^{0}}{d x^{0}}, \quad \varepsilon_{0}^{M} \triangleq \frac{d x^{M}}{d x^{0}}=b_{0}^{0} c_{0}^{M}=b_{0}^{A} c_{A}^{M}, \\
& c_{0}^{M} \triangleq \frac{d x^{M}}{d \xi^{0}}, \quad c_{0}^{0} \triangleq \frac{d x^{0}}{d \xi^{0}}, \quad \delta_{0}^{A} \triangleq \frac{d \xi^{A}}{d \xi^{0}}=c_{0}^{0} b_{0}^{A}=c_{0}^{M} b_{M}^{A} .
\end{aligned}
$$

Define $d \xi_{0} \triangleq \frac{d x^{0}}{d \xi^{0}} d x^{0}$ and $d x_{0} \triangleq \frac{d \xi^{0}}{d x^{0}} d \xi^{0}$, which induce $\frac{d}{d \xi_{0}}$ and $\frac{d}{d x_{0}}$, such that $\left\langle\frac{d}{d \xi_{0}}, d \xi_{0}\right\rangle=1$, $\left\langle\frac{d}{d x_{0}}, d x_{0}\right\rangle=1$. So we can also define

$$
\begin{aligned}
& \bar{b}_{A}^{0} \triangleq \frac{d \xi_{A}}{d x_{0}}, \quad \bar{b}_{0}^{0} \triangleq \frac{d \xi_{0}}{d x_{0}}, \quad \bar{\varepsilon}_{M}^{0} \triangleq \frac{d x_{M}}{d x_{0}}=\bar{b}_{0}^{0} \bar{c}_{M}^{0}=\bar{b}_{A}^{0} \bar{c}_{M}^{A}, \\
& \bar{c}_{M}^{0} \triangleq \frac{d x_{M}}{d \xi_{0}}, \quad \bar{c}_{0}^{0} \triangleq \frac{d x_{0}}{d \xi_{0}}, \quad \bar{\delta}_{A}^{0} \triangleq \frac{d \xi_{A}}{d \bar{\xi}_{0}}=\bar{c}_{0}^{0} \bar{b}_{A}^{0}=\bar{c}_{M}^{0} \bar{b}_{A}^{M} .
\end{aligned}
$$

They determine the following smooth functions on the entire $L$, similar to section 2.2:

$$
\begin{array}{ll}
B_{0}^{A}: L \rightarrow \mathbb{R}, \quad p \mapsto B_{0}^{A}(p) \triangleq\left(b_{f(p)}\right)_{0}^{A}(p), & C_{0}^{M}: L \rightarrow \mathbb{R}, p \mapsto C_{0}^{M}(p) \triangleq\left(c_{f(p)}\right)_{0}^{M}(p), \\
\bar{B}_{A}^{0}: L \rightarrow \mathbb{R}, \quad p \mapsto \bar{B}_{A}^{0}(p) \triangleq\left(\bar{b}_{f(p)}\right)_{A}^{0}(p), & \bar{C}_{M}^{0}: L \rightarrow \mathbb{R}, p \mapsto \bar{C}_{M}^{0}(p) \triangleq\left(\bar{c}_{f(p)}\right)_{M}^{0}(p), \\
B_{0}^{0}: L \rightarrow \mathbb{R}, \quad p \mapsto B_{0}^{0}(p) \triangleq\left(b_{f(p)}\right)_{0}^{0}(p), & C_{0}^{0}: L \rightarrow \mathbb{R}, p \mapsto C_{0}^{0}(p) \triangleq\left(c_{f(p)}\right)_{0}^{0}(p), \\
\bar{B}_{0}^{0}: L \rightarrow \mathbb{R}, \quad p \mapsto \bar{B}_{0}^{0}(p) \triangleq\left(\bar{b}_{f(p)}\right)_{0}^{0}(p), & \bar{C}_{0}^{0}: L \rightarrow \mathbb{R}, p \mapsto \bar{C}_{0}^{0}(p) \triangleq\left(\bar{c}_{f(p)}\right)_{0}^{0}(p) .
\end{array}
$$

For convenience, we still use the notations $\varepsilon$ and $\delta$, and have the following smooth functions.

$$
\begin{aligned}
& \varepsilon_{0}^{M} \triangleq B_{0}^{0} C_{0}^{M}=B_{0}^{A} C_{A}^{M}, \quad \delta_{0}^{A} \triangleq C_{0}^{0} B_{0}^{A}=C_{0}^{M} B_{M}^{A}, \quad G_{00} \triangleq B_{0}^{0} B_{0}^{0}=G_{M N} \varepsilon_{0}^{M} \varepsilon_{0}^{N} . \\
& \bar{\varepsilon}_{M}^{0} \triangleq \bar{B}_{0}^{0} \bar{C}_{M}^{0}=\bar{B}_{A}^{0} \bar{C}_{M}^{A}, \quad \bar{\delta}_{A}^{0} \triangleq \bar{C}_{0}^{0} \bar{B}_{A}^{0}=\bar{C}_{M}^{0} \bar{B}_{A}^{M}, \quad G^{00} \triangleq C_{0}^{0} C_{0}^{0}=G^{M N} \bar{\varepsilon}_{M}^{0} \bar{\varepsilon}_{N}^{0} \text {. }
\end{aligned}
$$

It is easy to verify that $d x_{0}=G_{00} d x^{0}$ and $\frac{d}{d x_{0}}=G^{00} \frac{d}{d x^{0}}$ are both true on $L$ by a simple calculation. 


\subsection{Evolution lemma}

We have the following two evolution lemmas. The affine connection representations of YangMills equation, energy-momentum equation and Dirac equation are dependent on them.

Definition 3.3.1. $\forall p \in L$, suppose $T_{p}(M)$ and $T_{p}(L)$ are tangent spaces, $T_{p}^{*}(M)$ and $T_{p}^{*}(L)$ are cotangent spaces. The regular imbedding $\pi: L \rightarrow M, q \mapsto q$ induces the tangent map and the cotangent map

$$
\begin{aligned}
& \pi_{*}: T_{p}(L) \rightarrow T_{p}(M), \quad\left[\gamma_{L}\right] \mapsto\left[\pi \circ \gamma_{L}\right], \\
& \pi^{*}: T_{p}^{*}(M) \rightarrow T_{p}^{*}(L), \quad d f \mapsto d(f \circ \pi) .
\end{aligned}
$$

Evidently, $\pi_{*}$ is an injection, and $\pi^{*}$ is a surjection. $\forall \frac{d}{d t_{L}} \in T_{p}(L), \frac{d}{d t} \in T_{p}(M), d f \in T_{p}^{*}(M), d f_{L} \in$ $T_{p}^{*}(L)$, if and only if

$$
\frac{d}{d t}=\pi_{*}\left(\frac{d}{d t_{L}}\right), \quad d f_{L}=\pi^{*}(d f)
$$

are true, we denote

$$
\frac{d}{d t} \cong \frac{d}{d t_{L}}, \quad d f \simeq d f_{L}
$$

Proposition 3.3.1. If $\frac{d}{d t} \cong \frac{d}{d t_{L}}$ and $d f \simeq d f_{L}$, then $\left\langle\frac{d}{d t}, d f\right\rangle=\left\langle\frac{d}{d t_{L}}, d f_{L}\right\rangle$.

Proof. We know the tangent vectors $\frac{d}{d t}$ and $\frac{d}{d t_{L}}$ are defined as equivalence classes $[\gamma]$ and $\left[\gamma_{L}\right]$ of parameter curves, the cotengent vectors $d f$ and $d f_{L}$ are defined as equivalence classes $[f]$ and $\left[f_{L}\right]$ of smooth functions.

$\frac{d}{d t}=\pi_{*}\left(\frac{d}{d t_{L}}\right)$ and $d f_{L}=\pi^{*}(d f)$ indicate that $\exists \gamma \in[\gamma], \gamma_{L} \in\left[\gamma_{L}\right], f \in[f], f_{L} \in\left[f_{L}\right]$ such that $\gamma=\pi \circ \gamma_{L}$ and $f_{L}=f \circ \pi$. Thus,

$$
\left\langle\frac{d}{d t}, d f\right\rangle=\left\langle\frac{d}{d t_{L}}, d f_{L}\right\rangle \Leftrightarrow\langle[\gamma],[f]\rangle=\left\langle\left[\gamma_{L}\right],\left[f_{L}\right]\right\rangle \Leftrightarrow \frac{d(f \circ \gamma)}{d t}=\frac{d\left(f_{L} \circ \gamma_{L}\right)}{d t},
$$

where

$$
f \circ \gamma=f \circ\left(\pi \circ \gamma_{L}\right), \quad f_{L} \circ \gamma_{L}=(f \circ \pi) \circ \gamma_{L} .
$$

Evidently we have $f \circ \gamma=f_{L} \circ \gamma_{L}$, and it makes $\left\langle\frac{d}{d t}, d f\right\rangle=\left\langle\frac{d}{d t_{L}}, d f_{L}\right\rangle$ true.

Proposition 3.3.2. The following conclusions are true.

$$
\left\{\begin{array} { l } 
{ w ^ { M } \frac { \partial } { \partial x ^ { M } } \cong w ^ { 0 } \frac { d } { d x ^ { 0 } } \Leftrightarrow w ^ { M } = w ^ { 0 } \varepsilon _ { 0 } ^ { M } , } \\
{ w _ { M } d x ^ { M } \simeq w _ { 0 } d x ^ { 0 } \Leftrightarrow w _ { M } \varepsilon _ { 0 } ^ { M } = w _ { 0 } , }
\end{array} \quad \left\{\begin{array}{l}
\bar{w}_{M} \frac{\partial}{\partial x_{M}} \cong \bar{w}_{0} \frac{d}{d x_{0}} \Leftrightarrow \bar{w}_{M}=\bar{w}_{0} \bar{\varepsilon}_{M}^{0}, \\
\bar{w}^{M} d x_{M} \simeq \bar{w}^{0} d x_{0} \Leftrightarrow \bar{w}^{M} \bar{\varepsilon}_{M}^{0}=\bar{w}^{0} .
\end{array}\right.\right.
$$

Proof. The following local discussion can also be applied to the entire manifold. Take the above left-hand side equations for example.

$$
\pi_{*}\left(\frac{d}{d x^{0}}\right)=\frac{d x^{M}}{d x^{0}} \frac{\partial}{\partial x^{M}} \Rightarrow \varepsilon_{0}^{M} \frac{\partial}{\partial x^{M}} \cong \frac{d}{d x^{0}} \Rightarrow w^{0} \varepsilon_{0}^{M} \frac{\partial}{\partial x^{M}} \cong w^{0} \frac{d}{d x^{0}} .
$$

We know $\pi_{*}$ is an injection, so $w^{M} \frac{\partial}{\partial x^{M}} \cong w^{0} \frac{d}{d x^{0}} \Leftrightarrow w^{M}=w^{0} \varepsilon_{0}^{M}$.

$$
\pi^{*}\left(d x^{M}\right)=\frac{d x^{M}}{d x^{0}} d x^{0} \Rightarrow d x^{M} \simeq \varepsilon_{0}^{M} d x^{0} \Rightarrow w_{M} d x^{M} \simeq w_{M} \varepsilon_{0}^{M} d x^{0} .
$$

We know $\pi^{*}$ is a surjection, so $w_{M} d x^{M} \simeq w_{0} d x^{0} \Leftrightarrow w_{M} \varepsilon_{0}^{M}=w_{0}$. 


\subsection{On-shell evolution as a gradient}

Definition 3.4.1. Let $T$ be an smooth $n$-order tensor field. The restriction on $\left(U, x^{M}\right)$ is $T \triangleq$ $t\left\{\frac{\partial}{\partial x} \otimes d x\right\}$, where $\left\{\frac{\partial}{\partial x} \otimes d x\right\}$ represents the tensor basis generated by several $\frac{\partial}{\partial x^{M}}$ and $d x^{M}$, and the tensor coefficients of $\mathbf{T}$ are concisely denoted by $t: U \rightarrow \mathbb{R}$.

Let $D$ be a simple connection. Consider $D \mathbf{T} \triangleq t_{; Q} d x^{Q} \otimes\left\{\frac{\partial}{\partial x} \otimes d x\right\}$. Denote

$$
D t \triangleq t_{; Q} d x^{Q}, \quad \nabla t \triangleq t_{; Q Q} \frac{\partial}{\partial x_{Q}} .
$$

$\forall p \in M$, the integral curve of $\nabla t$, that is $L_{p} \triangleq \varphi_{\nabla t, p}$, is a gradient line of $\mathbf{T}$. It can be seen later that the above gradient operator $\nabla$ characterizes the on-shell evolution.

For any evolution path $L$, let $U_{L} \triangleq U \cap L$. Denote $t_{L} \triangleq t_{U_{L}}$ and $t_{L ; 0} \triangleq t_{; Q} \varepsilon_{0}^{Q}$, as well as

$$
D_{L} t_{L} \triangleq t_{L ; 0} d x^{0}, \quad \nabla_{L} t_{L} \triangleq t_{L ; 0} \frac{d}{d x_{0}} .
$$

Proposition 3.4.1. The following conclusions are evidently true.

(i) $D t \simeq D_{L} t_{L}$ if and only if $L$ is an arbitrary evolution path.

(ii) $\nabla t \cong \nabla_{L} t_{L}$ if and only if $L$ is a gradient line of $\mathbf{T}$.

Definition 3.4.2. More generally, suppose there is a tensor $\mathbf{U} \triangleq u_{Q} d x^{Q} \otimes\left\{\frac{\partial}{\partial x} \otimes d x\right\}$. In such a notation, all the indices are concisely ignored except $Q . u_{Q} d x^{Q}$ uniquely determines a characteristic direction $u_{Q} \frac{\partial}{\partial x_{Q}}$.

If the system of 1-order linear partial differential equations $t_{; Q}=u_{Q}$ has a solution $t$, then it is true that $D t=u_{Q} d x_{Q}$ and $\nabla t=u_{Q} \frac{d}{d x_{Q}}$.

If it does not have a solution, we also denote the notations $D t \triangleq u_{Q} d x_{Q}$ and $\nabla t \triangleq u_{Q} \frac{d}{d x_{Q}}$ in form for convenience.

Thus, no matter whether $t_{; Q}=u_{Q}$ has a solution, as long as we take the evolution direction $[L]$ as $[L]=u_{Q} \frac{\partial}{\partial x_{Q}}$, the following conclusions are always true.

$$
D t \simeq D_{L} t_{L}, \quad \nabla t \cong \nabla_{L} t_{L},
$$

where $D_{L} t_{L} \triangleq u_{0} d x^{0}, \nabla_{L} t_{L} \triangleq u_{0} \frac{d}{d x_{0}}$ and $u_{0} \triangleq u_{Q} \varepsilon_{0}^{Q}$.

Remark 3.4.1. Now for any geometric property in the form of tensor $\mathbf{U}$, we are able to express its on-shell evolution in the form of $\nabla t$.

Next, two important on-shell evolutions are discussed in the following two sections. One is the on-shell evolution of the potential field of a reference-system. The other is the one that a general charge of a reference-system evolves in the potential field of another reference-system.

\subsection{On-shell evolution of potential field and affine connection representation of Yang-Mills equation}

The table I of the article[76] proposes a famous correspondence between gauge field terminologies and fibre bundle terminologies. However, it does not find out the corresponding mathematical object to the source $J_{\mu}^{K}$. In this section, we give an answer to this problem from the point of view of section 3.4, and show the affine connection representation of Yang-Mills equation. 
Suppose $f$ evolves in $g$ according to Definition 3.2.1, that is, $\forall p \in M$,

$$
\left(U, \xi^{A}\right) \stackrel{f(p)}{\longrightarrow}\left(U, x^{M}\right) \stackrel{g(p)}{\longleftarrow}\left(U, \zeta^{A}\right)
$$

We always take the following notations in the coordinate frame $\left(U, x^{M}\right)$.

(i) Let the simple connections of geometric manifolds $(M, f)$ and $(M, g)$ be $\Lambda_{N P}^{M}$ and $\Gamma_{N P}^{M}$, respectively. The colon ":" and semicolon ";" are used to express the covariant derivatives on $(M, f)$ and $(M, g)$, respectively, e.g.:

$$
u_{: P}^{Q}=\frac{\partial u^{Q}}{\partial x^{P}}+u^{H} \Lambda_{H P}^{Q}, \quad u_{; P}^{Q}=\frac{\partial u^{Q}}{\partial x^{P}}+u^{H} \Gamma_{H P}^{Q} .
$$

$\Lambda_{N P}^{M}$ is said to be the potential field of $f$, and $\Gamma_{N P}^{M}$ is said to be the potential field of $g$.

(ii) Let the coefficients of Riemannian curvatures of $(M, f)$ and $(M, g)$ be $K_{N P Q}^{M}$ and $R_{N P Q}^{M}$, respectively, i.e.

$$
\begin{aligned}
& K_{N P Q}^{M} \triangleq \frac{\partial \Lambda_{N Q}^{M}}{\partial x^{P}}-\frac{\partial \Lambda_{N P}^{M}}{\partial x^{Q}}+\Lambda_{N Q}^{H} \Lambda_{H P}^{M}-\Lambda_{N P}^{H} \Lambda_{H Q}^{M} \\
& R_{N P Q}^{M} \triangleq \frac{\partial \Gamma_{N Q}^{M}}{\partial x^{P}}-\frac{\partial \Gamma_{N P}^{M}}{\partial x^{Q}}+\Gamma_{N Q}^{H} \Gamma_{H P}^{M}-\Gamma_{N P}^{H} \Gamma_{H Q}^{M}
\end{aligned}
$$

(iii) The values of indices of internal space and external space are taken according to Definition 4.1.

Consider the evolution of $f$. Denote $K_{N P Q}^{M}: P \triangleq G^{P P^{\prime}} K_{N P Q: P^{\prime}}^{M}$. On an arbitrary evolution path $L$, we define

$$
\rho_{N 0}^{M} d x^{0} \triangleq \pi^{*}\left(K_{N P Q}^{M}: P d x^{Q}\right) \in T^{*}(L)
$$

Then, according to Definition 3.3.1 and the evolution lemma of Proposition 3.3.2, we obtain $\rho_{N 0}^{M}=K_{N P Q}^{M}: P \varepsilon_{0}^{Q}$ and

$$
K_{N P Q}^{M} \quad: P d x^{Q} \simeq \rho_{N 0}^{M} d x^{0}
$$

According to Definition 3.4.2, let the characteristic direction of $K_{N P Q}^{M}: P d x^{Q}$ be $\nabla t$. Then, according to Proposition 3.4.1, $\forall p \in L$, if and only if $\left[L_{p}\right]=\left.\nabla t\right|_{p}$, we have

$$
\left.\nabla t\right|_{p}=K_{N P Q}^{M}: P \frac{\partial}{\partial x_{Q}} \cong \rho_{N 0}^{M} \frac{d}{d x_{0}}=\left.\nabla_{L} t\right|_{p} .
$$

Applying the evolution lemma of Proposition 3.3.2 again, we obtain

$$
K_{N P Q}^{M}: P=\rho_{N 0}^{M} \bar{\varepsilon}_{Q}^{0}
$$

Denote $j_{N Q}^{M} \triangleq \rho_{N 0}^{M} \varepsilon_{Q}^{0}$, then if and only if $\left[L_{p}\right]=\left.\nabla t\right|_{p}$ we have

$$
K_{N P Q}^{M}: P=j_{N Q}^{M}
$$

which is said to be (affine) Yang-Mills equation of $f$. Thus, we have the following two results. 
(i) We obtain the mathematical origination of charge and current. We know that the evolution path $L$ is an imbedding submanifold of $M$. Thus, the charge $\rho_{N 0}^{M}$ originates from the pull-back $\pi^{*}$ from $M$ to $L$ for the curvature divergence, and the current $j_{N Q}^{M}$ originates from $\nabla t$ that is related to $\rho_{N 0}^{M}$. Besides, there is a further explanation in section 4.4.

(ii) We obtain the following proposition. This shows that Yang-Mills equation originates from a geometric property in the direction of $\nabla t$.

Proposition 3.5.1. Let $\nabla t$ be the characteristic direction of $K_{N P Q}^{M}{ }^{: P} d x^{Q}$. At any point $p$ on $M$, the Yang-Mills equation $K_{N P Q}^{M}: P=j_{N Q}^{M}$ holds if and only if the evolution direction $\left[L_{p}\right]=\left.\nabla t\right|_{p}$.

Definition 3.5.1. We speak of

$$
\rho_{M N}^{0} \triangleq G^{00} G_{M M^{\prime}} \rho_{N 0}^{M^{\prime}}
$$

as the field function of a general charge, or a charge for short.

\subsection{On-shell evolution of general charge and affine connection representation of mass, energy, momentum and action}

In order to be compatible with the affine connection representation of gauge fields, we also have to define mass, energy, momentum and action in the form associated to affine connection. We are going to show them in this section and section 4.4.

Let $F^{0} \triangleq \rho_{M N}{ }^{0} d x^{M} \otimes d x^{N}$. For the sake of simplicity, denote $\rho_{M N}{ }^{0}$ by $\rho_{M N}$ concisely. Let $D$ be the simple connection of $(M, g)$, then

$$
D F^{0} \triangleq D \rho_{M N} \otimes d x^{M} \otimes d x^{N}, \quad \nabla F^{0} \triangleq \nabla \rho_{M N} \otimes d x^{M} \otimes d x^{N},
$$

where $D \rho_{M N} \triangleq \rho_{M N ; Q} d x^{Q}$ and $\nabla \rho_{M N} \triangleq \rho_{M N ; Q} \frac{\partial}{\partial x_{Q}}$. According to Proposition 3.4.1, if and only if the evolution direction is taken as $[L]=\nabla \rho_{M N}$, we have

$$
D \rho_{M N} \simeq D_{L} \rho_{M N}, \quad \nabla \rho_{M N} \cong \nabla_{L} \rho_{M N}
$$

that is

$$
\rho_{M N ; Q} d x^{Q} \simeq \rho_{M N ; 0} d x^{0}, \quad \rho_{M N ; Q} \frac{\partial}{\partial x_{Q}} \cong \rho_{M N ; 0} \frac{d}{d x_{0}} .
$$

Definition 3.6.1. For more convenience, the notation $\rho_{M N}$ is further abbreviated as $\rho$. In affine connection representation, energy and momentum of $\rho$ are defined as

$$
\begin{aligned}
& E_{0} \triangleq \rho_{; 0} \triangleq \rho_{; Q} \varepsilon_{0}^{Q}, \quad p_{Q} \triangleq \rho_{; Q}, \quad H_{0} \triangleq \frac{d \rho}{d x^{0}}, \quad P_{Q} \triangleq \frac{\partial \rho}{\partial x^{Q}} \\
& E^{0} \triangleq \rho^{; 0} \triangleq \rho^{; Q} \bar{\varepsilon}_{Q}^{0}, \quad p^{Q} \triangleq \rho^{; Q},
\end{aligned}
$$

Proposition 3.6.1. Let the charge $\rho$ of $f$ evolves in $g$. At any point $p$ on $M$, the equation

$$
E_{0} E^{0}=p_{Q} p^{Q}
$$

holds if and only if the evolution direction $\left[L_{p}\right]=\left.\nabla \rho\right|_{p}$. Eq.(20) is the (affine) energy-momentum equation of $\rho$. 
Proof. According to the above discussion, $\forall p \in M,\left[L_{p}\right]=\left.\nabla \rho\right|_{p}$ is equivalent to

$$
p_{Q} d x^{Q} \simeq E_{0} d x^{0}, \quad p_{Q} \frac{\partial}{\partial x_{Q}} \cong E_{0} \frac{d}{d x_{0}} .
$$

Then due to Proposition 3.3.1 we obtain the directional derivative in the gradient direction $\nabla \rho$ :

$$
\left\langle p_{Q} \frac{\partial}{\partial x_{Q}}, p_{M} d x^{M}\right\rangle=\left\langle E_{0} \frac{d}{d x_{0}}, E_{0} d x^{0}\right\rangle,
$$

i.e. $G^{Q M} p_{Q} p_{M}=G^{00} E_{0} E_{0}$, or $p_{Q} p^{Q}=E_{0} E^{0}$.

Proposition 3.6.2. Let the charge $\rho$ of $f$ evolves in $g$. At any point $p$ on $M$, the equations

$$
p^{Q}=E^{0} \frac{d x^{Q}}{d x^{0}}, \quad p_{Q}=E_{0} \frac{d x_{Q}}{d x_{0}}
$$

hold if and only if the evolution direction $\left[L_{p}\right]=\left.\nabla \rho\right|_{p}$.

Proof. Due to the evolution lemma of Proposition 3.3.2, we immediately obtain Eq.(22) from Eq.(21).

Remark. According to Proposition 4.2.2, the light velocity $c=1$, therefore in the gradient direction $\nabla \rho$, the conclusion of Proposition 3.6.2 is consistent with the conventional

$$
p=m v .
$$

Thus, in affine connection representation, the energy-momentum equation and the conventional definition of momentum both originate from a geometric property in gradient direction.

Definition 3.6.2. Let $\mathcal{P}(b, a)$ be the totality of paths from $a$ to $b$. And suppose $L \in \mathcal{P}(b, a)$, and the evolution parameter $x^{0}$ satisfies $t_{a} \triangleq x^{0}(a)<x^{0}(b) \triangleq t_{b}$. The elementary affine action of $\rho$ is defined as

$$
\mathfrak{s}(L) \triangleq \int_{L} D \rho=\int_{L} p_{Q} d x^{Q}=\int_{t_{a}}^{t_{b}} E_{0} d x^{0} .
$$

Thus, $\delta \mathfrak{s}(L)=0$ if and only if $L$ is a gradient line of $\rho$.

Specially, in the case where $g$ is orthogonal, we can also define action in the following way.

On $(M, g)$ let there be Dirac algebras $\gamma^{M}$ and $\gamma_{N}$ such that

$$
\gamma^{M} \gamma^{N}+\gamma^{N} \gamma^{M}=2 G^{M N}, \quad \gamma_{M} \gamma_{N}+\gamma_{N} \gamma_{M}=2 G_{M N}, \quad \gamma_{M} \gamma^{M}=1 .
$$

In a gradient direction of $\rho$, from Eq.(20) we obtain that

$$
\begin{aligned}
p_{Q} p^{Q}=E_{0} E^{0} & \Leftrightarrow \rho_{; Q} \rho^{; Q}=\rho_{; 0} \rho^{; 0} \\
& \Leftrightarrow G^{P Q} \rho_{; P} \rho_{; Q}=G^{00} \rho_{; 0} \rho_{; 0} \\
& \Leftrightarrow\left(\gamma^{P} \gamma^{Q}+\gamma^{Q} \gamma^{P}\right) \rho_{; P} \rho_{; Q}=2 \rho_{; 0} \rho_{; 0} \\
& \Leftrightarrow\left(\gamma^{P} \rho_{; P}\right)\left(\gamma^{Q} \rho_{; Q}\right)+\left(\gamma^{Q} \rho_{; Q}\right)\left(\gamma^{P} \rho_{; P}\right)=2 \rho_{; 0} \rho_{; 0} \\
& \Leftrightarrow\left(\gamma^{P} \rho_{; P}\right)^{2}=\left(\rho_{; 0}\right)^{2} .
\end{aligned}
$$


Take $\gamma^{P} \rho_{; P}=\rho_{; 0}$ without loss of generality, then, in the gradient direction of $\rho$ we have

$$
\gamma^{P} \rho_{; P} d x^{0}=\rho_{; 0} d x^{0}=\varepsilon_{0}^{P} \rho_{; P} d x^{0}=D \rho .
$$

So we can define the following (orthogonal affine) action of $\rho$.

$$
s(L) \triangleq \int_{L}\left(\gamma^{P} \rho_{; P} d x^{0}+D \rho\right)=\int_{t_{a}}^{t_{b}}\left(\gamma^{P} \rho_{; P}+\varepsilon_{0}^{P} \rho_{; P}\right) d x^{0}=\int_{t_{a}}^{t_{b}}\left(\gamma^{P} \rho_{; P}+E_{0}\right) d x^{0} .
$$

Remark 3.6.1 and Remark 4.5.1 explain the rationality of this definition. We have $s(L)=2 \mathfrak{s}(L)$ in the gradient direction of $\rho$, so $\mathfrak{s}(L)$ and $s(L)$ are consistent.

Remark 3.6.1. In the Minkowski coordinate frame of Definition 4.2.1, the evolution parameter $x^{0}$ is replaced by $\tilde{x}^{\tau}$, then there still exists a concept of gradient direction $\tilde{\nabla} \tilde{\rho}$. Correspondingly,

$$
\mathfrak{s}(L) \triangleq \int_{L} D \rho=\int_{L} p_{Q} d x^{Q}=\int_{t_{a}}^{t_{b}} E_{0} d x^{0}, \quad s(L)=\int_{t_{a}}^{t_{b}}\left(\gamma^{P} \rho_{; P}+E_{0}\right) d x^{0}
$$

present as

$$
\tilde{\mathfrak{s}}(L) \triangleq \int_{L} \tilde{D} \tilde{\rho}=\int_{L} \tilde{p}_{\mu} d \tilde{x}^{\mu}=\int_{\tau_{a}}^{\tau_{b}} \tilde{m}_{\tau} d \tilde{x}^{\tau}, \quad \tilde{s}(L)=\int_{\tau_{a}}^{\tau_{b}}\left(\gamma^{\mu} \tilde{\rho}_{; \mu}+\tilde{m}_{\tau}\right) d \tilde{x}^{\tau},
$$

where $\tilde{m}_{\tau}$ is the rest-mass and $\tilde{x}^{\tau}$ is the proper-time.

\subsection{Affine connection representation of interaction}

First, we define the following notations.

$$
\begin{array}{ll}
{\left[\rho \Gamma_{G}\right] \triangleq \frac{\partial \rho}{\partial x^{G}}-\rho_{; G} \triangleq \frac{\partial \rho_{M N}}{\partial x^{G}}-\rho_{M N ; G}=\rho_{M H} \Gamma_{N G}^{H}+\rho_{H N} \Gamma_{M G}^{H},} & {\left[\rho \Gamma^{Q}\right] \triangleq G^{G Q}\left[\rho \Gamma_{G}\right],} \\
{\left[\rho \Gamma_{0}\right] \triangleq \frac{d \rho}{d x^{0}}-\rho_{; 0} \triangleq \frac{d \rho_{M N}}{d x^{0}}-\rho_{M N ; 0}=\rho_{M H} \Gamma_{N 0}^{H}+\rho_{H N} \Gamma_{M 0}^{H},} & {\left[\rho \Gamma^{0}\right] \triangleq G G^{00}\left[\rho \Gamma_{0}\right],} \\
{\left[\rho R_{P Q}\right] \triangleq \rho_{M H} R_{N P Q}^{H}+\rho_{H N} R_{M P Q}^{H},} & {\left[\rho E_{P Q}\right] \triangleq\left[\rho \Gamma_{Q}\right]_{; P}-\left[\rho \Gamma_{P}\right]_{; Q},} \\
{\left[\rho B_{P Q}\right] \triangleq \rho_{M H}\left(\frac{\partial \Gamma_{N Q}^{H}}{\partial x^{P}}-\frac{\partial \Gamma_{N P}^{H}}{\partial x^{Q}}\right)+\rho_{H N}\left(\frac{\partial \Gamma_{M Q}^{H}}{\partial x^{P}}-\frac{\partial \Gamma_{M P}^{H}}{\partial x^{Q}}\right),} & {\left[\rho F_{P Q}\right] \triangleq \frac{\partial\left[\rho \Gamma_{Q}\right]}{\partial x^{P}}-\frac{\partial\left[\rho \Gamma_{P}\right]}{\partial x^{Q}}}
\end{array}
$$

Then, through some calculations, we can obtain that

$$
\begin{array}{ll}
F_{P} \triangleq \frac{d p_{P}}{d x^{0}}=\frac{\partial E_{0}}{\partial x^{P}}-p_{Q} \frac{\partial \varepsilon_{0}^{Q}}{\partial x^{P}}+\left[\rho F_{P Q}\right] \varepsilon_{0}^{Q}, & {\left[\rho F_{P Q}\right]=\left[\rho E_{P Q}\right],} \\
f_{P} \triangleq p_{P ; 0}=E_{0 ; P}-p_{Q} \varepsilon_{0 ; P}^{Q}+\left[\rho R_{P Q}\right] \varepsilon_{0}^{Q}, & {\left[\rho B_{P Q}\right]=\left[\rho R_{P Q}\right],}
\end{array}
$$

which are the affine connection representations of general Lorentz force equations. See Discussion 4.4.1 for further illustrations.

Furthermore, the affine connection representation of the conservation of energy-momentum can base on the above result to be constructed. We need to take $Y_{M N}$ such that $Y_{M N} ; M=$ $-G^{00}\left(E_{0 ; N}-p_{Q} \varepsilon_{0 ; N}^{Q}+\left[\rho R_{N Q}\right] \varepsilon_{0}^{Q}\right)-\varepsilon_{M}^{0 ; M} p_{N}$. And let $W_{M N} \triangleq E_{0} \varepsilon_{M}^{0} \varepsilon_{N}^{0}, T_{M N} \triangleq W_{M N}+Y_{M N}$. According to Proposition 3.6.2, $E_{0} \bar{\varepsilon}_{N}^{0}=p_{N}$ in the gradient direction of $\rho$. Then

$$
W_{M N} ; M=\left(E_{0} \bar{\varepsilon}_{M}^{0} \bar{\varepsilon}_{N}^{0}\right)^{; M}=\left(\bar{\varepsilon}_{M}^{0} p_{N}\right)^{; M}=p_{N} ; M \bar{\varepsilon}_{M}^{0}+\bar{\varepsilon}_{M}^{0 ; M} p_{N}=p_{N} ; 0+\bar{\varepsilon}_{M}^{0 ; M} p_{N}=-Y_{M N}{ }^{; M} .
$$

Thus, $\left(W_{M N}+Y_{M N}\right)^{; M}=0$, that is $T_{M N} ; M=0$. 


\subsection{Inversion transformation in affine connection representation}

In affine conection representation, $C P T$ inversion is interpreted as a total inversion of coordinates and metrics. Let $i, j=1,2,3$ and $m, n=4,5, \cdots, \mathfrak{D}$.

Let the local coordinate representation of reference-system $k$ be $x^{\prime j}=-\delta_{i}^{j} x^{i}, x^{\prime n}=\delta_{m}^{n} x^{m}$, then parity inversion can be represented as

$$
P \triangleq L_{k}: x^{i} \rightarrow-x^{i}, x^{m} \rightarrow x^{m} .
$$

Let the local coordinate representation of reference-system $h$ be $x^{\prime j}=\delta_{i}^{j} x^{i}, x^{\prime n}=-\delta_{m}^{n} x^{m}$, then charge conjugate inversion can be represented as

$$
C \triangleq L_{h}: x^{i} \rightarrow x^{i}, x^{m} \rightarrow-x^{m} .
$$

Time coordinate invesion can be represented as

$$
T_{0}: x^{0} \rightarrow-x^{0} .
$$

Total inversion of coordinates can be represented as

$$
C P T_{0}: x^{Q} \rightarrow-x^{Q}, x^{0} \rightarrow-x^{0} .
$$

The positive or negative sign of metric marks two opposite directions of evolution. Let $N$ be a closed submanifold of $M$, and let its metric be $d x^{(N)}$. Denote the totality of closed submanifolds of $M$ by $\mathfrak{B}(M)$, then total inversion of metrics can be expressed as

$$
T^{(M)} \triangleq \prod_{N \in \mathfrak{B}(M)}\left(d x^{(N)} \rightarrow-d x^{(N)}\right) .
$$

Denote time inversion by

$$
T \triangleq T^{(M)} T_{0}
$$

then the joint transformation of the total inversion of coordinates $C P T_{0}$ and the total inversion of metrics $T^{(M)}$ is

$$
\left(C P T_{0}\right)\left(T^{(M)}\right)=C P T .
$$

Summerize the above discussions, then we have

$$
\begin{aligned}
& C P T_{0}: x^{Q} \rightarrow-x^{Q}, x^{0} \rightarrow-x^{0}, d x^{Q} \rightarrow d x^{Q}, d x^{0} \rightarrow d x^{0}, \\
& T^{(M)}: x^{Q} \rightarrow x^{Q}, x^{0} \rightarrow x^{0}, d x^{Q} \rightarrow-d x^{Q}, d x^{0} \rightarrow-d x^{0}, \\
& C P T: x^{Q} \rightarrow-x^{Q}, x^{0} \rightarrow-x^{0}, d x^{Q} \rightarrow-d x^{Q}, d x^{0} \rightarrow-d x^{0} .
\end{aligned}
$$

The $C P T$ invariance in affine connection representation is very clear. Concretely, on $(M, g)$ we consider the $C P T$ transformation acting on $g$. Denote $s \triangleq \int_{L} D \rho$ and $D_{P} e^{i s} \triangleq\left(\frac{\partial}{\partial x^{P}}-i\left[\rho \Gamma_{P}\right]\right) e^{i s}$, then through simple calculations we obtain that

$$
C P T: D \rho \rightarrow D \rho, \quad D_{P} e^{i s} \rightarrow-D_{P} e^{i s} .
$$

Remark 3.8.1. In quantum mechanics there is a complex conjugation in the time inversion of wave function $T: \psi(x, t) \rightarrow \psi^{*}(x,-t)$. In affine connection representation, we know the complex conjugation is a straightforward mathematical result of the total inversion of metrics $T^{(M)}$. 


\subsection{Two dual descriptions of gradient direction field}

Discussion 3.9.1. Let $X$ and $Y$ be non-vanishing smooth tangent vector fields on the manifold $M$. And let $L_{Y}$ be the Lie derivative operator induced by the one-parameter group of diffeomorphism $\varphi_{Y}$. Then, according to a well-known theorem[77], we obtain the Lie derivative equation

$$
[X, Y]=L_{Y} X .
$$

Suppose $\forall p \in M, Y(p)$ is a unit-length vector, i.e. $\|Y(p)\|=1$. Let the parameter of $\varphi_{Y}$ be $x^{0}$. Then, on the evolution path $L \triangleq \varphi_{Y, p}$, we have

$$
Y \cong \frac{d}{d x^{0}} .
$$

Thus, Eq.(29) can also be represented as

$$
[X, Y]=\frac{d}{d x^{0}} X .
$$

On the other hand, $\forall d f \in T(M)$ and $d f_{L} \triangleq \pi^{*}(d f)$, due to (30) and Proposition 3.3.1 we have $\langle Y, d f\rangle=\left\langle\frac{d}{d x^{0}}, d f_{L}\right\rangle$, that is

$$
Y f=\frac{d}{d x^{0}} f_{L} .
$$

Definition 3.9.1. Let $H \triangleq\|\nabla \rho\|^{-1} \nabla \rho=\varepsilon_{0}^{M} \frac{\partial}{\partial x^{M}} \cong \frac{d}{d x^{0}}$. It is evident that $\forall p \in M,\|H(p)\|=1$. If and only if taking $Y=H$, we speak of (31) and (32) as real-valued (affine) Heisenberg equation and (affine) Schrödinger equation, respectively, that is

$$
[X, H]=\frac{d}{d x^{0}} X, \quad H f=\frac{d}{d x^{0}} f_{L} .
$$

Discussion 3.9.2. The above two equations both describe the gradient direction field, and thereby reflect on-shell evolution. Such two dual descriptions of gradient direction show the real-valued affine connection representation of Heisenberg picture and Schrödinger picture.

It is not hard to find out several different kinds of complex-valued representations of gradient direction. For examples, one is the affine Dirac equation in section 4.5, another is as follows.

Let $\psi \triangleq f e^{i s_{L}}$, where it is fine to take either $s_{L} \triangleq s(L)$ or $s_{L} \triangleq \mathfrak{s}(L)$ from Definition 3.6.2. According to Eq.(33), it is easy to obtain on $L$, that

$$
[X, H]=\frac{d}{d x^{0}} X, \quad H \psi=\frac{d \psi}{d x^{0}} .
$$

This is consistent with the conventional Heisenberg equation and Schrödinger equation (taking the natural units that $\hbar=1, c=1$ )

$$
[X,-i H]=\frac{\partial}{\partial t} X, \quad-i H \psi=\frac{\partial \psi}{\partial t}
$$

and they have a coordinate correspondence

$$
\frac{\partial}{\partial\left(i x^{k}\right)} \leftrightarrow \frac{\partial}{\partial x^{k}}, \quad \frac{\partial}{\partial t} \leftrightarrow \frac{d}{d x^{0}} .
$$


Due to Discussion 4.3.1 we know that $\frac{\partial}{\partial t} \leftrightarrow \frac{d}{d x^{0}}$ originates from the difference that the evolution parameter is $x^{\tau}$ or $x^{0}$. Due to Discussion 4.3.2 we know that the imaginary unit $i$ originates from the difference between the regular coordinates $x^{1}, x^{2}, x^{3}, x^{\tau}$ and the Minkowski coordinates $x^{1}, x^{2}, x^{3}, x^{0}$. That is to say, the regular coordinates satisfy

$$
\left(d x^{0}\right)^{2}=\left(d x^{1}\right)^{2}+\left(d x^{2}\right)^{2}+\left(d x^{3}\right)^{2}+\left(d x^{\tau}\right)^{2},
$$

and the Minkowski coordinates satisfy

$$
\left(d x^{\tau}\right)^{2}=\left(d x^{0}\right)^{2}-\left(d x^{1}\right)^{2}-\left(d x^{2}\right)^{2}-\left(d x^{3}\right)^{2}=\left(d x^{0}\right)^{2}+\left(d\left(i x^{1}\right)\right)^{2}+\left(d\left(i x^{2}\right)\right)^{2}+\left(d\left(i x^{3}\right)\right)^{2} .
$$

This causes the appearance of the imaginary unit $i$ in the correspondence

$$
i x^{k} \leftrightarrow x^{k} .
$$

So Eq.(34) and Eq.(35) have exactly the same essence, and their differences only come from different coordinate representations.

The differences between coordinate representations have nothing to do with the geometric essence and the physical essence. We notice that the value of a gradient direction is dependent on the intrinsic geometry, but independent of that the equations are real-valued or complex-valued. Therefore, it is unnecessary for us to confine to such algebraic forms as real-valued or complexvalued forms, but we should focus on such geometric essence as gradient direction.

The advantage of complex-valued form is that it is convenient for describing the coherent superposition of the propagator. However, this is independent of the above discussions, and we are going to discuss it in section 3.10 .

\subsection{Quantum evolution as a distribution of gradient directions}

From Proposition 3.6.1 we see that, in affine connection representation, the classical on-shell evolution is described by gradient direction. Then, naturally, quantum evolution should be described by the distribution of gradient directions.

The distribution of gradient directions on a geometric manifold $(M, g)$ is effected by the bending shape of $(M, g)$, in other words, the distribution of gradient directions can be used to reflect the shape of $(M, g)$. This is the way that the quantum theory in affine connection representation describes physical reality.

In order to know the full picture of physical reality, it is necessary to fully describe the shape of the geometric manifold. For a single observation:

(1) It is the reference-system, not a point, that is used to describe the physical reality, so the coordinate of an individual point is not enough to fully describe the location information about the physical reality.

(2) Through a single observation of momentum, we can only obtain information about an individual gradient direction, this cannot reflect the full picture of the shape of the geometric manifold.

Quantum evolution provides us with a guarantee that we can obtain the distribution of gradient directions through multiple observations, so that we can describe the full picture of the shape of the geometric manifold.

Next, we are going to carry out strict mathematical descriptions for the quantum evolution in affine connetion representation. 
Definition 3.10.1. Let $\rho$ be a geometric property on $M$, such as a charge of $f$. Then $H \triangleq \nabla \rho$ is a gradient direction field of $\rho$ on $(M, g)$.

Let $\mathfrak{T}$ be the totality of all flat transformations $L_{[k]}$ defined in section 2.3. $\forall T \in \mathfrak{T}$, the flat transformation $T: f \mapsto T f$ induces a transformation $T^{*}: \rho \mapsto T^{*} \rho$. Denote

$$
|\rho| \triangleq\left\{\rho_{T} \triangleq T^{*} \rho \mid T \in \mathfrak{T}\right\}, \quad|H| \triangleq\left\{H_{T} \triangleq \nabla \rho_{T} \mid T \in \mathfrak{T}\right\} .
$$

$\forall a \in M$, the restriction of $|H|$ at $a$ are denoted by $|H(a)| \triangleq\left\{H_{T}(a) \mid T \in \mathfrak{T}\right\}$.

We say $|H|$ is the total distribution of the gradient direction field $H$.

$\forall T \in \mathfrak{T}$, we say $H_{T}$ is a position distribution of gradient directions in momentum representation.

$\forall a \in M$, we say $|H(a)|$ is a momentum distribution of gradient directions in position representation.

Remark 3.10.1. When $T$ is fixed, $H_{T}$ can reflect the shape of $(M, g)$. When $a$ is fixed, $|H(a)|$ can reflect the shape of $(M, g)$.

However, when $T$ and $a$ are both fixed, $H_{T}(a)$ is a fixed individual gradient direction, which cannot reflect the shape of $(M, g)$. In other words, if the momentum $p_{T}$ and the position $x_{a}$ of $\rho$ are both definitely observed, the physical reality $g$ would be unknowable, therefore this is unacceptable. This is the embodiment of quantum uncertainty in affine connection representation.

Definition 3.10.2. Let $\varphi_{H}$ be the one-parameter group of diffeomorphisms corresponding to $H$. The parameter of $\varphi_{H}$ is $x^{0} . \forall a \in M$, according to Definition 3.2.1, let $\varphi_{H, a}$ be the evolution path through $a$, such that $\varphi_{H, a}(0)=a . \forall t \in \mathbb{R}$, denote

$$
\varphi_{|H|, a} \triangleq\left\{\varphi_{X, a}|X \in| H \mid\right\}, \quad \varphi_{|H|, a}(t) \triangleq\left\{\varphi_{X, a}(t)|X \in| H \mid\right\} .
$$

$\forall \Omega \subseteq \mathfrak{T}$, we also denote $\left|H_{\Omega}\right| \triangleq\left\{H_{T} \mid T \in \Omega\right\} \subseteq|H|$ and

$$
\varphi_{\left|H_{\Omega}\right|, a} \triangleq\left\{\varphi_{X, a}|X \in| H_{\Omega} \mid\right\} \subseteq \varphi_{|H|, a}, \quad \varphi_{\left|H_{\Omega}\right|, a}(t) \triangleq\left\{\varphi_{H, a}(t)|X \in| X_{\Omega} \mid\right\} \subseteq \varphi_{|H|, a}(t) .
$$

$\forall a \in M$, the restriction of $\left|H_{\Omega}\right|$ at $a$ are denoted by $\left|H_{\Omega}(a)\right| \triangleq\left\{H_{T}(a) \mid T \in \Omega\right\}$.

Remark 3.10.2. At the beginning $t=0$, intuitively, the gradient directions $|H(a)|$ of $|\rho|$ start from $a$ and point to all directions around $a$ uniformly. If $(M, g)$ is not flat, when evolving to a certain time $t>0$, the distribution of gradient directions on $\varphi_{|H|, a}(t)$ is no longer as uniform as beginning. The following definition precisely characterizes this kind of ununiformity.

Definition 3.10.3. Let the transformation $L_{g^{-1}}$ act on $g$, then we obtain the trivial $e \triangleq L_{g^{-1}}(g)$. Now $(M, g)$ is sent to a flat $(M, e)$, and the gradient direction field $|H|$ of $|\rho|$ on $(M, g)$ is sent to a gradient direction field $|O|$ of $|\rho|$ on $(M, e)$. Correspondingly, $\forall t \in \mathbb{R}, \varphi_{|H|, a}(t)$ is sent to $\varphi_{|O|, a}(t)$. In a word, $L_{g^{-1}}$ induces the following two maps:

$$
g_{*}^{-1}:|H| \rightarrow|O|, \quad g_{* *}^{-1}: \varphi_{|H|, a} \rightarrow \varphi_{|O|, a} .
$$

$\forall T \in \mathfrak{T}$, deonte $\mathfrak{N} \triangleq\{N \in \mathfrak{T} \mid \operatorname{det} N=\operatorname{det} T\}$. Due to $\mathfrak{T} \cong G L(\mathfrak{D}, \mathbb{R})$, let $\mathfrak{U}$ be a neighborhood of $T$, with respect to the topology of $G L(\mathfrak{D}, \mathbb{R})$.

Take $\Omega=\mathfrak{N} \cap \mathfrak{U}$, then

$$
\left|O_{\Omega}\right|=g_{*}^{-1}\left(\left|H_{\Omega}\right|\right), \quad \varphi_{\left|O_{\Omega}\right|, a}=g_{* *}^{-1}\left(\varphi_{\left|H_{\Omega}\right|, a}\right) .
$$


Let $\mu$ be a Borel measure on the manifold $M . \forall t \in \mathbb{R}$, we know

$$
\varphi_{\left|H_{\mathfrak{N}}\right|, a}(t) \simeq \varphi_{\left|O_{\mathfrak{N}}\right|, a}(t) \simeq \mathbb{S}^{\mathfrak{D}-1}
$$

Thus, $\varphi_{\left|H_{\Omega}\right|, a}(t) \subseteq \varphi_{\left|H_{\mathfrak{N}}\right|, a}(t)$ and $\varphi_{\left|O_{\Omega}\right|, a}(t) \subseteq \varphi_{\left|O_{\mathfrak{N}}\right|, a}(t)$ are Borel sets, so they are measurable. Denote

$$
\mu_{a}\left(\varphi_{\left|H_{\Omega}\right|, a}(t)\right) \triangleq \mu\left(g_{* *}^{-1}\left(\varphi_{\left|H_{\Omega}\right|, a}(t)\right)\right)=\mu\left(\varphi_{\left|O_{\Omega}\right|, a}(t)\right) .
$$

When $\mathfrak{U} \rightarrow T$, we have $\Omega \rightarrow T,\left|H_{\Omega}\right| \rightarrow H_{T},\left|H_{\Omega}(a)\right| \rightarrow H_{T}(a)$, and $\varphi_{\left|H_{\Omega}\right|, a}(t) \rightarrow b \triangleq \varphi_{H_{T}, a}(t)$.

For the sake of simplicity, denote $L \triangleq \varphi_{H_{T}, a}$. Thus, we have $a=L(0), b=L(t)$, and denote $p_{a} \triangleq\left[L_{a}\right]=H_{T}(a), p_{b} \triangleq\left[L_{b}\right]=H_{T}(b)$.

Because $\mu_{a}$ is absolutely continuous with respect to $\mu$, Radon-Nikodym theorem[78] ensures the existence of the following limit. The Radon-Nikodym derivative

$$
W_{L}(b, a) \triangleq \frac{d \mu_{a}}{d \mu_{b}} \triangleq \lim _{\mathfrak{U} \rightarrow T} \frac{\mu_{a}\left(\varphi_{\left|H_{\Omega}\right|, a}(t)\right)}{\mu\left(\varphi_{\left|H_{\Omega}\right|, a}(t)\right)}=\lim _{\mathfrak{U} \rightarrow T} \frac{\mu\left(g_{* *}^{-1}\left(\varphi_{\left|H_{\Omega}\right|, a}(t)\right)\right)}{\mu\left(\varphi_{\left|H_{\Omega}\right|, a}(t)\right)}=\lim _{\mathfrak{U} \rightarrow T} \frac{\mu\left(\varphi_{\left|O_{\Omega}\right|, a}(t)\right)}{\mu\left(\varphi_{\left|H_{\Omega}\right|, a}(t)\right)}
$$

is called the distribution density of $|H|$ along $L$ in position representation.

On a neighborhood $U$ of $a, \forall T \in \mathfrak{T}$, denote the normal section of $H_{T}(a)$ by $N_{H_{T}, a}$, that is

$$
N_{H_{T}, a} \triangleq\left\{n \in U \mid H_{T}(a) \cdot(n-a)=0\right\}, \quad N_{H_{T}, a}(t) \triangleq\left\{\varphi_{H_{T}, x}(t) \mid x \in N_{H_{T}, a}\right\} .
$$

Thus, $N_{H_{T}, a}=N_{H_{T}, a}(0)$ and $N_{H_{T}, b} \triangleq N_{H_{T}, a}(t)$. If $U \rightarrow a$, we have $N_{H_{T}, a} \rightarrow a$ and $N_{H_{T}, a}(t) \rightarrow$ $b \triangleq \varphi_{H_{T}, a}(t)$. The Radon-Nikodym derivative

$$
Z_{L}(b, a) \triangleq \frac{d \mu(a)}{d \mu(b)} \triangleq \lim _{U \rightarrow a} \frac{\mu\left(N_{H_{T}, a}\right)}{\mu\left(N_{H_{T}, b}\right)}=\lim _{U \rightarrow a} \frac{\mu\left(N_{H_{T}, a}\right)}{\mu\left(N_{H_{T}, a}(t)\right)}
$$

is called the distribution density of $|H|$ along $L$ in momentum representation.

In a word, $W_{L}(b, a)$ and $Z_{L}\left(p_{b}, p_{a}\right)$ describe the density of the gradient lines that are adjacent to $b$ in two different ways. They have the following evident property.

Proposition 3.10.1. Let $L$ be a gradient line. $\forall a, b, c \in L$ such that $L\left(x_{a}^{0}\right)=a, L\left(x_{b}^{0}\right)=b$, $L\left(x_{c}^{0}\right)=c$ and $x_{b}^{0}>x_{c}^{0}>x_{a}^{0}$, then

$$
W_{L}(b, a)=W_{L}(b, c) W_{L}(c, a), \quad Z_{L}(b, a)=Z_{L}(b, c) Z_{L}(c, a) .
$$

Proof. Directly compute

$$
\begin{aligned}
& W_{L}(b, c) W_{L}(c, a)=\frac{d \mu_{a}}{d \mu_{c}} \frac{d \mu_{c}}{d \mu_{b}}=\frac{d \mu_{a}}{d \mu_{b}}=W_{L}(b, a), \\
& Z_{L}(b, c) Z_{L}(c, a)=\frac{d \mu(a)}{d \mu(c)} \frac{d \mu(c)}{d \mu(b)}=\frac{d \mu(a)}{d \mu(b)}=Z_{L}(b, a) .
\end{aligned}
$$

Definition 3.10.4. If $L$ is a gradient line of some $\rho^{\prime} \in|\rho|$, we also say $L$ is a gradient line of $|\rho|$.

Remark 3.10.3. For any $a$ and $b$, it anyway makes sense to discuss the gradient line of $|\rho|$ from $a$ to $b$. It is because even if the gradient line of $\rho$ starting from $a$ does not pass through $b$, it just only 
needs to carry out a certain flat transformation $T$ defined in section 2.3 to obtain a $\rho^{\prime} \triangleq T_{*} \rho$, thus the gradient line of $\rho^{\prime}$ starting from $a$ can just exactly pass through $b$. Due to $\rho, \rho^{\prime} \in|\rho|$, we do not distinguish them, it is just fine to uniformly use $|\rho|$. Intuitively speaking, when $|\rho|$ takes two different initial momentums, $|\rho|$ presents as $\rho$ and $\rho^{\prime}$, respectively.

Discussion 3.10.1. With the above preparations, we obtain a new way to describe the construction of the propagator strictly.

For any path $L$ that starts at $a$ and ends at $b$, we denote $\|L\| \triangleq \int_{L} d x^{0}$ concisely. Let $\mathcal{P}(b, a)$ be the totality of all the paths from $a$ to $b$. Denote

$$
\mathcal{P}\left(b, x_{b}^{0} ; a, x_{a}^{0}\right) \triangleq\left\{L \mid L \in \mathcal{P}(b, a),\|L\|=x_{b}^{0}-x_{a}^{0}\right\} .
$$

$\forall L \in \mathcal{P}\left(b, x_{b}^{0} ; a, x_{a}^{0}\right)$, we can let $L\left(x_{a}^{0}\right)=a$ and $L\left(x_{b}^{0}\right)=b$ without loss of generality. Thus, $\mathcal{P}\left(b, x_{b}^{0} ; a, x_{a}^{0}\right)$ is the totality of all the paths from $L\left(x_{a}^{0}\right)=a$ to $L\left(x_{b}^{0}\right)=b$.

Abstractly, the propagator is defined as the Green function of the evolution equation. Concretely, the propagator still needs a constructive definition. One method is the Feynman path integral[79]

$$
K\left(b, x_{b}^{0} ; a, x_{a}^{0}\right) \triangleq \int_{\mathcal{P}\left(b, x_{b}^{0} ; a, x_{a}^{0}\right)} e^{i s} d L .
$$

However, there are so many redundant paths in $\mathcal{P}\left(b, x_{b}^{0} ; a, x_{a}^{0}\right)$ that: (i) it is difficult to generally define a measure $d L$ on $\mathcal{P}\left(b, x_{b}^{0} ; a, x_{a}^{0}\right)$, (ii) it may cause unnecessary infinities when carrying out some calculations.

In order to solve this problem, we try to reduce the scope of summation from $\mathcal{P}\left(b, x_{b}^{0} ; a, x_{a}^{0}\right)$ to $H\left(b, x_{b}^{0} ; a, x_{a}^{0}\right)$, where $H\left(b, x_{b}^{0} ; a, x_{a}^{0}\right)$ is the totality of all the gradient lines of $|\rho|$ from $L\left(x_{a}^{0}\right)=a$ to $L\left(x_{b}^{0}\right)=b$. Thus, the (38) is turned into

$$
K\left(b, x_{b}^{0} ; a, x_{a}^{0}\right)=\int_{H\left(b, x_{b}^{0} ; a, x_{a}^{0}\right)} \Psi(L) e^{i s} d L .
$$

We notice that as long as we take the probability amplitude $\Psi(L)$ of the gradient line $L$ such that $[\Psi(L)]^{2}=W_{L}(b, a)$ in position representation, or take $[\Psi(L)]^{2}=Z_{L}(b, a)$ in momentum representation, it can exactly be consistent with the Copenhagen interpretation. This provides the following new constructive definition for the propagator.

Definition 3.10.5. Suppose $|\rho|$ is defined as Definition 3.10.1, and denote $H \triangleq \nabla \rho$.

Let $\mathcal{L}(b, a)$ be the totality of all the gradient lines of $|\rho|$ from $a$ to $b$. Denote

$$
H\left(b, x_{b}^{0} ; a, x_{a}^{0}\right) \triangleq\left\{L \mid L \in \mathcal{L}(b, a),\|L\|=x_{b}^{0}-x_{a}^{0}\right\} .
$$

Let $\mathcal{L}\left(p_{b}, p_{a}\right)$ be the totality of all the gradient lines of $|\rho|$, whose starting-direction is $p_{a}$ and ending-direction is $p_{b}$. Denote

$$
H\left(p_{b}, x_{b}^{0} ; p_{a}, x_{a}^{0}\right) \triangleq\left\{L \mid L \in \mathcal{L}\left(p_{b}, p_{a}\right),\|L\|=x_{b}^{0}-x_{a}^{0}\right\} .
$$

Let $d L$ be a Borel measure on $H\left(b, x_{b}^{0} ; a, x_{a}^{0}\right)$. In consideration of Remark 4.5.1, we let $s$ be the affine action $s(L)$ in Definition 3.6.2. We say the intrinsic geometric property

$$
K\left(b, x_{b}^{0} ; a, x_{a}^{0}\right) \triangleq \int_{H\left(b, x_{b}^{0} ; a, x_{a}^{0}\right)} \sqrt{W_{L}(b, a)} e^{i s} d L
$$


is the propagator of $|\rho|$ from $\left(a, x_{a}^{0}\right)$ to $\left(b, x_{b}^{0}\right)$ in position representation. If we let $d L$ be a Borel measure on $H\left(p_{b}, x_{b}^{0} ; p_{a}, x_{a}^{0}\right)$, then we say

$$
\mathcal{K}\left(p_{b}, x_{b}^{0} ; p_{a}, x_{a}^{0}\right) \triangleq \int_{H\left(p_{b}, x_{b}^{0} ; p_{a}, x_{a}^{0}\right)} \sqrt{Z_{L}(b, a)} e^{i s} d L
$$

is the propagator of $|\rho|$ from $\left(p_{a}, x_{a}^{0}\right)$ to $\left(p_{b}, x_{b}^{0}\right)$ in momentum representation.

Discussion 3.10.2. Now (39) and (40) are strictly defined, but the Feynman path integral (38) has not been possessed of a strict mathematical definition until now. This makes it impossible at present to obtain (e.g. in position representation) a strict mathematical proof of

$$
\int_{H\left(b, x_{b}^{0} ; a, x_{a}^{0}\right)} \sqrt{W_{L}(b, a)} e^{i s} d L=\int_{\mathcal{P}\left(b, x_{b}^{0} ; a, x_{a}^{0}\right)} e^{i s} d L .
$$

We notice that the distribution densities $W_{L}(b, a)$ and $Z_{L}(b, a)$ of gradient directions establish an association between probability interpretation and geometric interpretation of quantum evolution. Therefore, we can base on probability interpretation to intuitively consider both sides of " $="$ as the same thing.

Discussion 3.10.3. The quantization methods of QFT are successful, and they are also applicable in affine connection representation for orthogonal $f$ and $g$, but in this paper we do not discuss them. We try to propose some more ideas for general $f$ and $g$ to understand the quantization of field in affine connection representation.

(1) If we take

$$
\mathfrak{s}=\int_{L} D \rho=\int_{L} p_{Q} d x^{Q}=\int_{L} E_{0} d x^{0}
$$

according to Definition 3.6.2, where $D$ is the simple connection of $(M, g)$, then consider the distribution of $H \triangleq \nabla \rho$, we know that

$K\left(b, x_{b}^{0} ; a, x_{a}^{0}\right) \triangleq \int_{\nabla \rho\left(b, x_{b}^{0} ; a, x_{a}^{0}\right)} \sqrt{W_{L}(b, a)} e^{i \mathfrak{s}} d L, \mathcal{K}\left(p_{b}, x_{b}^{0} ; p_{a}, x_{a}^{0}\right) \triangleq \int_{\nabla \rho\left(p_{b}, x_{b}^{0} ; p_{a}, x_{a}^{0}\right)} \sqrt{Z_{L}(b, a)} e^{i \mathfrak{s}} d L$

describe the quantization of energy-momentum. Every gradient line in $\nabla \rho\left(b, x_{b}^{0} ; a, x_{a}^{0}\right)$ corresponds to a set of eigenvalues of energy and momentum.

(2) If we take

$$
\mathfrak{s}=\int_{L} D t=\int_{L} K_{N P Q}^{M}: P d x^{Q}=\int_{L} \rho_{N 0}^{M} d x^{0},
$$

according to section 3.5, where $D$ is the simple connection of $(M, f)$, then consider the distribution of $H \triangleq \nabla t$, we know that

$K\left(b, x_{b}^{0} ; a, x_{a}^{0}\right) \triangleq \int_{\nabla t\left(b, x_{b}^{0} ; a, x_{a}^{0}\right)} \sqrt{W_{L}(b, a)} e^{i \mathfrak{s}} d L, \mathcal{K}\left(p_{b}, x_{b}^{0} ; p_{a}, x_{a}^{0}\right) \triangleq \int_{\nabla t\left(p_{b}, x_{b}^{0} ; p_{a}, x_{a}^{0}\right)} \sqrt{Z_{L}(b, a)} e^{i \mathfrak{s}} d L$

describe the quantizations of charge and current. It should be emphasized that this is not the quantization of the energy-momentum of the field, but the quantization of the field itself, which presents as quantized (e.g. discrete) charges and currents. 


\section{Affine connection representation of gauge fields in classical spacetime}

The new framework established in section 3 is discussed in the $\mathfrak{D}$-dimensional general coordinate $x^{M}$, which is more general than the $(1+3)$-dimensional conventional Minkowski coordinate $x^{\mu}$. This is different from the extra dimensions in Kaluza-Klein theory and string theory.

(1) The evolution parameter of the $\mathfrak{D}$-dimensional general coordinate $x^{M}(M=1,2, \cdots, \mathfrak{D})$ is $x^{0}$. That is to say, $\left(d x^{0}\right)^{2}=\sum_{M=1}^{\mathfrak{D}}\left(d x^{M}\right)^{2}=\sum_{i=1}^{3}\left(d x^{i}\right)^{2}+\left(d x^{\tau}\right)^{2}$, and the parameter equation of an evolution path $L$ is represented as $x^{M}=x^{M}\left(x^{0}\right)$.

(2) The evolution parameter of the $(1+3)$-dimensional Minkowski coordinate $x^{\mu}(\mu=0,1,2,3)$ is $x^{\tau}$. That is to say, $\left(d x^{\tau}\right)^{2}=\left(d x^{0}\right)^{2}-\sum_{i=1}^{3}\left(d x^{i}\right)^{2}$, and the parameter equation of $L$ is represented as $x^{\mu}=x^{\mu}\left(x^{\tau}\right)$.

(3) $x^{\mu}$ is strictly constructed from $x^{M}$ in section 4.2. So $x^{M}$ is more fundamental than $x^{\mu}$.

Definition 4.1. Suppose $M=P \times N, \mathfrak{D} \triangleq \operatorname{dim} M$ and $r \triangleq \operatorname{dim} P=3$. According to Definition 3.1.1, we have a submanifold of external space $P$ and a submanifold of internal space $N$. $P$ inherits coordinates $\left\{\xi^{s}\right\}\left\{x^{i}\right\}$ from $M$, and $N$ inherits coordinates $\left\{\xi^{a}\right\}\left\{x^{m}\right\}$ from $M$. The values of indices are specified as follows.

(1) Complete indices are $A, B, C, D=1,2, \cdots, \mathfrak{D} ; \quad M, N, P, Q=1,2, \cdots, \mathfrak{D}$.

(2) Indices of $\xi$ are $s, t, u, v=1,2, \cdots, r ; \quad a, b, c, d=r+1, r+2, \cdots, \mathfrak{D}$.

(3) Indices of $x$ are $i, j, k, l=1,2, \cdots, r ; \quad m, n, p, q=r+1, r+2, \cdots, \mathfrak{D}$.

(4) Regular and Minkowski indices of $\xi$ are $S, T, U, V=1,2, \cdots, r, \tau ; \alpha, \beta, \gamma, \delta=0,1,2, \cdots, r$.

(5) Regular and Minkowski indices of $x$ are $I, J, K, L=1,2, \cdots, r, \tau ; \mu, \nu, \rho, \sigma=0,1,2, \cdots, r$.

\subsection{Existence and uniqueness of classical spacetime submanifold}

Definition 4.1.1. Let there be a smooth tangent vector field $X$ on $(M, f)$. If $\forall p \in M, X(p)=$ $\left.b^{A} \frac{\partial}{\partial \xi^{A}}\right|_{p}=\left.c^{M} \frac{\partial}{\partial x^{M}}\right|_{p}$ satisfies that $b^{a}$ are not all zero and $c^{m}$ are not all zero, then we say $X$ is internal-directed. For any evolution path $L \triangleq \varphi_{X, p}$, we also say $L$ is internal-directed.

Proposition 4.1.1. Suppose $M=P \times N$ and $X$ is a smooth tangent vector field on $M$. Fix a point $o \in M$. If $X$ is internal-directed, then:

(1) There exist a unique $(r+1)$-dimensional regular submanifold $\gamma: \tilde{M} \rightarrow M, p \mapsto p$ and a unique smooth tangent vector field $\tilde{X}$ on $\tilde{M}$ such that:

(i) $P \times\{o\}$ is a closed submanifold of $\tilde{M}$.

(ii) The tangent map $\gamma_{*}: T(\tilde{M}) \rightarrow T(M)$ satisfies that $\forall q \in \tilde{M}, \gamma_{*}: \tilde{X}(q) \mapsto X(q)$.

Such an $\tilde{M}$ is called a classical spacetime submanifold determined by $X$ through $o$.

(2) Let

$$
\varphi_{X}: M \times \mathbb{R} \rightarrow M
$$

be the one-parameter group of diffeomorphisms corresponding to $X$, and

$$
\varphi_{\tilde{X}}: \tilde{M} \times \mathbb{R} \rightarrow \tilde{M}
$$

the one-parameter group of diffeomorphisms corresponding to $\tilde{X}$. Thus, we have

$$
\varphi_{\tilde{X}}=\left.\varphi_{X}\right|_{\tilde{M} \times \mathbb{R}} .
$$


Proof. We take two steps to prove.

Step 1. To construct $\tilde{M}$. We define a closed submanifold $P \times\{o\}$ on $M$ through $o$ via the parameter equation $x^{m}=x_{o}^{m}$. Then let $\varphi_{X}: M \times \mathbb{R} \rightarrow M$ be the one-parameter group of diffeomorphisms corresponding to $X$. Restrict $\varphi_{X}$ to $P \times\{o\}$ and we obtain

$$
\left.\varphi_{X}\right|_{P \times\{o\}}: P \times\{o\} \times\{t\} \mapsto P^{\prime} \times\left\{o^{\prime}\right\},
$$

where points $o$ and $o^{\prime}$ are on the same orbit $L_{o} \triangleq \varphi_{X, o} . P \times\{o\}$ and $P^{\prime} \times\left\{o^{\prime}\right\}$ are both homeomorphic to $P$. In this sense we do not distinguish $P$ and $P^{\prime}$, we have

$$
\left.\varphi_{X}\right|_{P \times\{o\} \times \mathbb{R}}: P \times\{o\} \times \mathbb{R} \rightarrow P \times L_{o} .
$$

Then consider all of such $\{o\}$ on the entire orbit $L_{o}$, and we obtain a map

$$
\left.\varphi_{X}\right|_{P \times L_{o} \times \mathbb{R}}: P \times L_{o} \times \mathbb{R} \rightarrow P \times L_{o} .
$$

Denote $\tilde{M} \triangleq P \times L_{o}$. So we know that $\tilde{M}$ is determined by $X$ and the fixed point $o$, hence it is unique. Thus,

$$
\left.\varphi_{X}\right|_{\tilde{M} \times \mathbb{R}}: \tilde{M} \times \mathbb{R} \rightarrow \tilde{M}
$$

constitutes a one-parameter group of diffeomorphisms on $\tilde{M}$.

Step 2. To construct $\gamma: \tilde{M} \rightarrow M$ and $\tilde{X}$. Because $X$ is internal-directed, the restriction of $X$ to $L_{o} \triangleq \varphi_{X, o}: \mathbb{R} \rightarrow M$ is non-vanishing everywhere and $L_{o}$ is an injection. The image set of $L_{o}$ can also be denoted by $L_{o} . \forall t \in \mathbb{R}, q \triangleq \varphi_{X, o}(t) \in L_{o}$, we can define a closed submanifold $N_{q}$ on $M$ through $q$ via the parameter equation $x^{i}=x_{q}^{i}$, and $N_{q}$ is homeomorphic to $N$. Due to the one-to-one correspondence between $q$ and $N_{q}, L_{o} \rightarrow N$ is a regular imbedding. Furthermore:

(1) $\gamma: P \times L_{o} \rightarrow P \times N$ is a regular imbedding, that is, $\gamma: \tilde{M} \rightarrow M$. Hence the tangent map $\gamma_{*}: T(\tilde{M}) \rightarrow T(M)$ is an injection. Therefore, the smooth tangent vector $\tilde{X}$ which satisfies $\forall q \in \tilde{M}, \gamma_{*}: \tilde{X}(q) \mapsto X(q)$ is uniquely defined by $X$ via $\gamma_{*}^{-1}$.

(2) We notice that $o \in \tilde{M}$, hence $L_{o} \triangleq \varphi_{X, o}$ is an orbit of $\left.\varphi_{X}\right|_{\tilde{M} \times \mathbb{R}}$. In consideration of that $L_{o}$ uniquely determines $\gamma$, and $\gamma$ uniquely determines $\gamma_{*}$, and $\gamma_{*}^{-1}$ uniquely determines $\tilde{X}$, so finally $\tilde{X}$ is uniquely determined by $\left.\varphi_{X}\right|_{\tilde{M} \times \mathbb{R}}$. Thus, we have $\varphi_{\tilde{X}}=\left.\varphi_{X}\right|_{\tilde{M} \times \mathbb{R}}$.

Remark 4.1.1. The above proposition constructs a $(3+1)$-dimensional submanifold of $M$, that is $\tilde{M}$. We know $\tilde{M}$ inherits a part of intrinsic geometric properties of $M$, so $\tilde{M}$ describes the physical properties in the classical $(3+1)$-dimensional spacetime. It should be noticed that:

(1) $\tilde{M}$ is not independent of $M$, but a regular submanifold of $M$.

(2) Not all of the intrinsic geometric properties of $M$ are inherited by $\tilde{M}$. The physical properties that are reflected by $\tilde{M}$ are not complete.

(3) The correspondence between $\tilde{X}$ and the restriction of $X$ to $\tilde{M}$ is one-to-one. For convenience, next we are not going to distinguish the notations $X$ and $\tilde{X}$ on $\tilde{M}$, but uniformly denote them by $X$.

(4) An arbitrary path $\tilde{L}: T \rightarrow \tilde{M}, t \mapsto p$ on $\tilde{M}$ uniquely corresponds to a path $L \triangleq \gamma \circ \tilde{L}$ : $T \rightarrow M, t \mapsto p$ on $M$. Evidently the image sets of $L$ and $\tilde{L}$ are the same, that is, $L(T)=\tilde{L}(T)$. For convenience, later we are not going to distinguish the notations $L$ and $\tilde{L}$ on $\tilde{M}$, but uniformly denote them by $L$. 


\subsection{Gravitational field on classical spacetime submanifold}

Proposition 4.2.1. Let there be a geometric manifold $(M, f)$ and its classical spacetime submanifold $\tilde{M}$. And let $L \triangleq \varphi_{\tilde{X}, a}$ be an evolution path on $\tilde{M}$. Suppose $p \in L$ and $U$ is a coordinate neighborhood of $p$. According to Defnition 3.2.2, suppose the $f(p)$ on $U$ and the $f_{L}(p)$ on $U_{L} \triangleq U \cap L$ satisfy that

$$
f(p), f_{L}(p): \xi^{A}=\xi^{A}\left(x^{M}\right)=\xi^{A}\left(x^{0}\right), \quad \xi^{0}=\xi^{0}\left(x^{0}\right) .
$$

Thus, according to the notations in Definition 4.1, it is true that:

(1) There exists a unique local reference-system $\tilde{f}(p)$ on $\tilde{U} \triangleq U \cap \tilde{M}$ such that

$$
\tilde{f}(p), \tilde{f}_{L}(p): \xi^{U}=\xi^{U}\left(x^{K}\right)=\xi^{U}\left(x^{0}\right), \quad \xi^{0}=\xi^{0}\left(x^{0}\right)
$$

and $\left(d x^{\tau}\right)^{2}=\sum_{m=r+1}^{\mathfrak{D}}\left(d x^{m}\right)^{2},\left(d \xi^{\tau}\right)^{2}=\sum_{a=r+1}^{\mathfrak{D}}\left(d \xi^{a}\right)^{2}$.

(2) If $L$ is internal-directed, then the above coordinate frames $\left(\tilde{U}, \xi^{U}\right)$ and $\left(\tilde{U}, x^{K}\right)$ of $\tilde{f}(p)$ uniquely determine the coordinate frames $\left(\tilde{U}, \tilde{\xi}^{\alpha}\right)$ and $\left(\tilde{U}, \tilde{x}^{\mu}\right)$ such that

$$
\tilde{f}(p), \tilde{f}_{L}(p): \tilde{\xi}^{\alpha}=\tilde{\xi}^{\alpha}\left(\tilde{x}^{\mu}\right)=\tilde{\xi}^{\alpha}\left(\tilde{x}^{\tau}\right), \quad \tilde{\xi}^{\tau}=\tilde{\xi}^{\tau}\left(\tilde{x}^{\tau}\right)
$$

and the coordinates satisfy

$$
\tilde{\xi}^{s}=\xi^{s}, \quad \tilde{\xi}^{\tau}=\xi^{\tau}, \quad \tilde{\xi}^{0}=\xi^{0}, \quad \tilde{x}^{i}=x^{i}, \quad \tilde{x}^{\tau}=x^{\tau}, \quad \tilde{x}^{0}=x^{0} .
$$

That is to say, $\tilde{f}(p), \tilde{f}_{L}(p)$ have two coordinate representations: (42) and (43).

Proof. (1) According to the proof of the previous proposition, $L$ is a regular submanifold of $N$. Let the metrics on $N$ be

$$
\left(d x^{\tau}\right)^{2}=\sum_{m=r+1}^{\mathfrak{D}}\left(d x^{m}\right)^{2}, \quad\left(d \xi^{\tau}\right)^{2}=\sum_{a=r+1}^{\mathfrak{D}}\left(d \xi^{a}\right)^{2} .
$$

Review Definition 3.2.2 and we know the regular imbedding $\pi: L \rightarrow N, q \mapsto q$ induces the parameter equations $x^{m}=x_{\tau}^{m}\left(x^{\tau}\right)$ and $\xi^{a}=\xi_{\tau}^{a}\left(\xi^{\tau}\right)$ of $L$. Then substitute them into $f(p), f_{L}(p)$ and we obtain

$$
\begin{aligned}
\xi^{A}=\xi^{A}\left(x^{M}\right)=\xi^{A}\left(x^{0}\right) & \Leftrightarrow\left\{\begin{array}{l}
\xi^{u}=\xi^{u}\left(x^{k}, x_{\tau}^{m}\left(x^{\tau}\right)\right)=\xi_{L}^{u}\left(x^{0}\right) \\
\xi_{\tau}^{a}\left(\xi^{\tau}\right)=\xi^{a}\left(x^{k}, x_{\tau}^{m}\left(x^{\tau}\right)\right)=\xi_{L}^{a}\left(x^{0}\right)
\end{array}\right. \\
& \Leftrightarrow\left\{\begin{array}{l}
\xi^{u}=\xi^{u}\left(x^{k}, x_{\tau}^{m}\left(x^{\tau}\right)\right)=\xi_{L}^{u}\left(x^{0}\right) \\
\xi^{\tau}=\left(\xi_{\tau}^{a}\right)^{-1} \circ \xi^{a}\left(x^{k}, x_{\tau}^{m}\left(x^{\tau}\right)\right)=\left(\xi_{\tau}^{a}\right)^{-1} \circ \xi_{L}^{a}\left(x^{0}\right)
\end{array}\right. \\
& \Leftrightarrow\left\{\begin{array}{l}
\xi^{u}=\xi^{u}\left(x^{k}, x^{\tau}\right)=\xi_{L}^{u}\left(x^{0}\right) \\
\xi^{\tau}=\xi^{\tau}\left(x^{k}, x^{\tau}\right)=\xi_{L}^{\tau}\left(x^{0}\right)
\end{array}\right. \\
& \Leftrightarrow \xi^{U}=\xi^{U}\left(x^{K}\right)=\xi_{L}^{U}\left(x^{0}\right), \text { abbreviated to } \xi^{U}=\xi^{U}\left(x^{K}\right)=\xi^{U}\left(x^{0}\right) .
\end{aligned}
$$

(2) As same as the above, we also obtain $x^{K}=x^{K}\left(\xi^{U}\right)=x^{K}\left(\xi^{0}\right)$. The relation between two parameters $x^{0}$ and $x^{\tau}$ of $L$ can be expressed as $x^{\tau}=x_{L}^{\tau}\left(\xi^{0}\left(x^{0}\right)\right)$, and the relation between two 
parameters $\xi^{0}$ and $\xi^{\tau}$ can be expressed as $\xi^{\tau}=\xi_{L}^{\tau}\left(x^{0}\left(\xi^{0}\right)\right)$. Substitute them into $\tilde{f}(p), \tilde{f}_{L}(p)$, then

$$
\begin{aligned}
& \xi^{U}=\xi^{U}\left(x^{K}\right)=\xi^{U}\left(x^{0}\right) \Leftrightarrow\left\{\begin{array}{l}
\xi^{u}=\xi^{u}\left(x^{k}, x_{L}^{\tau}\left(\xi^{0}\left(x^{0}\right)\right)\right)=\xi_{L}^{u}\left(x^{0}\left(\left(x_{L}^{\tau}\right)^{-1}\left(x^{\tau}\right)\right)\right) \\
\xi_{L}^{\tau}\left(x^{0}\left(\xi^{0}\right)\right)=\xi^{\tau}\left(x^{k}, x_{L}^{\tau}\left(\xi^{0}\left(x^{0}\right)\right)\right)=\xi_{L}^{\tau}\left(x^{0}\left(\left(x_{L}^{\tau}\right)^{-1}\left(x^{\tau}\right)\right)\right) \\
\xi^{0}\left(\xi_{L}^{\tau-1}\left(\xi^{\tau}\right)\right)=\left(x_{L}^{\tau}\right)^{-1}\left(x^{\tau}\right)
\end{array}\right. \\
& \Leftrightarrow\left\{\begin{array}{l}
\xi^{u}=\xi^{u}\left(x^{k}, x_{L}^{\tau}\left(\xi^{0}\left(x^{0}\right)\right)\right)=\xi_{L}^{u}\left(x^{0}\left(\left(x_{L}^{\tau}\right)^{-1}\left(x^{\tau}\right)\right)\right) \\
\xi^{0}=\xi^{0}\left(\left(\xi_{L}^{\tau}\right)^{-1}\left(\xi^{\tau}\left(x^{k}, x_{L}^{\tau}\left(\xi^{0}\left(x^{0}\right)\right)\right)\right)\right)=\left(x_{L}^{\tau}\right)^{-1}\left(x^{\tau}\right), \\
\xi^{\tau}=\xi_{L}^{\tau}\left(x^{0}\left(\left(x_{L}^{\tau}\right)^{-1}\left(x^{\tau}\right)\right)\right)
\end{array}\right.
\end{aligned}
$$

which can be abbreviated to

$$
\left\{\begin{array}{l}
\xi^{u}=\tilde{\xi}^{u}\left(x^{k}, x^{0}\right)=\tilde{\xi}_{L}^{u}\left(x^{\tau}\right) \\
\xi^{0}=\tilde{\xi}^{0}\left(x^{k}, x^{0}\right)=\tilde{\xi}_{L}^{0}\left(x^{\tau}\right) . \\
\xi^{\tau}=\tilde{\xi}^{\tau}\left(x^{\tau}\right)
\end{array}\right.
$$

Denote

$$
\tilde{\xi}^{s} \triangleq \xi^{s}, \quad \tilde{\xi}^{\tau} \triangleq \xi^{\tau}, \quad \tilde{\xi}^{0} \triangleq \xi^{0}, \quad \tilde{x}^{i} \triangleq x^{i}, \quad \tilde{x}^{\tau} \triangleq x^{\tau}, \quad \tilde{x}^{0} \triangleq x^{0},
$$

hence we have $\tilde{\xi}^{\alpha}=\tilde{\xi}^{\alpha}\left(\tilde{x}^{\mu}\right)=\tilde{\xi}_{L}^{\alpha}\left(\tilde{x}^{\tau}\right), \quad \tilde{\xi}^{\tau}=\tilde{\xi}^{\tau}\left(\tilde{x}^{\tau}\right)$, abbreviated to

$$
\tilde{\xi}^{\alpha}=\tilde{\xi}^{\alpha}\left(\tilde{x}^{\mu}\right)=\tilde{\xi}^{\alpha}\left(\tilde{x}^{\tau}\right), \quad \tilde{\xi}^{\tau}=\tilde{\xi}^{\tau}\left(\tilde{x}^{\tau}\right) .
$$

Definition 4.2.1. Due to the above local reference-system $\tilde{f}(p)$, we thereby have a reference-system

$$
\tilde{f}: \tilde{M} \rightarrow R E F_{\tilde{M}}, p \mapsto \tilde{f}(p) \in R E F_{p} .
$$

In fact, $\tilde{f}(p)$ is a reference system in conventional sense. We say $\tilde{f}$ is a classical spacetime reference-system on $\tilde{M}$, and $(\tilde{M}, \tilde{f})$ is a gravitational manifold.

$\left(\tilde{U}, \xi^{U}\right)$ and $\left(\tilde{U}, x^{K}\right)$ are called regular coordinate frames, meanwhile $\left(\tilde{U}, \tilde{\xi}^{\alpha}\right)$ and $\left(\tilde{U}, \tilde{x}^{\mu}\right)$ are called Minkowski coordinate frames.

Remark 4.2.1. $\tilde{f}$ is uniquely determined by $f$, and $\tilde{f}$ encapsulates the internal space of $f$. Although $(\tilde{M}, \tilde{f})$ can reflect the intrinsic geometric properties of external space of $(M, f)$, it cannot totally reflect all the intrinsic geometric properties of internal space of $(M, f)$. There is a further illustration in section 4.6.

Definition 4.2.2. Suppose we have a geometric manifold $(\tilde{M}, \tilde{g}) . F_{\tilde{g}}$ is a transformation induced by $\tilde{g}$.

(1) According to Discussion 4.3.2, it is easy to know that $\tilde{\delta}_{\alpha \beta} \tilde{B}_{\mu}^{\alpha} \tilde{B}_{\nu}^{\beta}=\tilde{\varepsilon}_{\mu \nu}$ if and only if $d \tilde{\zeta}^{\tau}=d \tilde{x}^{\tau}$. Thus, we denote $d \tilde{\zeta}^{\tau}$ and $d \tilde{x}^{\tau}$ uniformly by $d \tau$, and say $\tilde{g}$ is orthogonal.

(2) If the semi-metrics $\tilde{B}_{\mu}^{\alpha}$ and $\tilde{C}_{\alpha}^{\mu}$ of $\tilde{g}$ are constants on $\tilde{M}$, then we say $\tilde{g}$ is flat.

(3) If $\tilde{g}$ is both orthogonal and flat, then we say $\tilde{g}$ is an inertial-system, $F_{\tilde{g}}$ is a Lorentz transformation, and $(\tilde{M}, \tilde{g})$ is the Minkowski spacetime. 
Proposition 4.2.2. Let $L$ be a path on $\tilde{M}$, and $\tilde{U}$ a coordinate neighborhood. Denote $\tilde{U}_{L} \triangleq \tilde{U} \cap L$, and $c \triangleq\left|d x^{i} / d x^{0}\right|$. Suppose $L$ satisfies that $\forall q \in \tilde{U}_{L}$, the tangent vector $\left[L_{q}\right] \in T_{q}(M)$ is not internal-directed. Then, it is true that:

(i) we have $c=1$ on path $\tilde{U}_{L}$.

(ii) $c=1$ remains unchanged under orthogonal transformations.

(iii) $c=1$ remains unchanged under Lorentz transformations.

Proof. (i) In $\left(\tilde{U}, x^{K}\right), \tilde{U}_{L}$ can be described by equations $x^{i}=x^{i}\left(x^{0}\right)$ and $x^{\tau}=$ const with respect to the parameter $x^{0}$. We notice that $x^{\tau}=$ const, so there does not exist an equation of $\tilde{U}_{L}$ with respect to the parameter $\tilde{x}^{\tau}$ in Minkowski coordinate frame $\left(\tilde{U}, \tilde{x}^{\mu}\right)$. Therefore, we always have $d \tilde{x}^{\tau}=d x^{\tau}=0$ on $\tilde{U}_{L}$. Thus,

$$
c=\left|d x^{i} / d x^{0}\right|=\left| \pm d x^{i} / \sqrt{\left(d x^{i}\right)^{2}+\left(d x^{\tau}\right)^{2}}\right|=\left| \pm d x^{i} / d x^{i}\right|=1 .
$$

(ii) According to Definition 4.2.2, an orthogonal transformation satisfies $d \tilde{\zeta}^{\tau}=d \tilde{x}^{\tau}=0$, hence

$$
c^{\prime}=\left|d \zeta^{s} / d \zeta^{0}\right|=\left| \pm d \zeta^{s} / d \zeta^{s}\right|=1=c .
$$

(iii) It is naturally true for the Lorentz transformation as an orthogonal one. This conclusion reflects the principle of constancy of light velocity.

\subsection{Mathematical conversion between two coordinate representations}

Discussion 4.3.1. According to Definition 3.2.2, suppose the semi-metrics of $(\tilde{M}, \tilde{f})$ with respect to the regular coordinate $x^{K}$ are $B_{I}^{S}$ and $C_{S}^{I}$, such that

$$
\begin{aligned}
& d \xi^{S}=B_{I}^{S} d x^{I} \simeq B_{0}^{S} d x^{0}, \quad d x^{I}=C_{S}^{I} d \xi^{S} \simeq C_{0}^{I} d \xi^{0}, \\
& C_{0}^{I} \frac{\partial}{\partial x^{I}} \cong C_{0}^{0} \frac{d}{d x^{0}}=\frac{d}{d \xi^{0}}, \quad B_{0}^{S} \frac{\partial}{\partial \xi^{S}} \cong B_{0}^{0} \frac{d}{d \xi^{0}}=\frac{d}{d x^{0}} .
\end{aligned}
$$

The semi-metrics of $(\tilde{M}, \tilde{f})$ with respect to the Minkowski coordinate $\tilde{x}^{\mu}$ are $\tilde{B}_{\mu}^{\alpha}$ and $\tilde{C}_{\alpha}^{\mu}$, such that

$$
\begin{array}{ll}
d \tilde{\xi}^{\alpha}=\tilde{B}_{\mu}^{\alpha} d \tilde{x}^{\mu} \simeq \tilde{B}_{\tau}^{\alpha} d \tilde{x}^{\tau}, \quad & d \tilde{x}^{\mu}=\tilde{C}_{\alpha}^{\mu} d \tilde{\xi}^{\alpha} \simeq \tilde{C}_{\tau}^{\mu} d \tilde{\xi}^{\tau}, \\
\tilde{C}_{\tau}^{\mu} \frac{\partial}{\partial \tilde{x}^{\mu}} \cong \tilde{C}_{\tau}^{\tau} \frac{d}{d \tilde{x}^{\tau}}=\frac{d}{d \tilde{\xi}^{\tau}}, \quad \tilde{B}_{\tau}^{\alpha} \frac{\partial}{\partial \tilde{\xi}^{\alpha}} \cong \tilde{B}_{\tau}^{\tau} \frac{d}{d \tilde{\xi}^{\tau}}=\frac{d}{d \tilde{x}^{\tau}} .
\end{array}
$$

Applying the chain rule of differentiation, it is not hard to obtain the following relations between the regular semi-metrics and the Minkowski semi-metrics via the formula (44).

$$
\begin{aligned}
& \tilde{B}_{i}^{s}=-B_{i}^{s}, \quad \tilde{B}_{\tau}^{s}=B_{\tau}^{s}, \quad \tilde{B}_{0}^{s}=B_{0}^{s}, \quad \tilde{C}_{s}^{i}=-C_{s}^{i}, \quad \tilde{C}_{\tau}^{i}=C_{\tau}^{i}, \quad \tilde{C}_{0}^{i}=C_{0}^{i}, \\
& \tilde{B}_{i}^{0}=-\frac{B_{i}^{\tau}}{\delta_{0}^{\tau}}, \quad \tilde{B}_{\tau}^{0}=\frac{B_{\tau}^{\tau}}{\delta_{0}^{\tau}}, \quad \tilde{B}_{0}^{0}=\frac{B_{0}^{\tau}}{\delta_{0}^{\tau}}, \quad \tilde{C}_{s}^{0}=-\frac{C_{s}^{\tau}}{\varepsilon_{0}^{\tau}}, \quad \tilde{C}_{\tau}^{0}=\frac{C_{\tau}^{\tau}}{\varepsilon_{0}^{\tau}}, \quad \tilde{C}_{0}^{0}=\frac{C_{0}^{\tau}}{\varepsilon_{0}^{\tau}}, \\
& \tilde{C}_{\tau}^{i}=\frac{C_{0}^{i}}{\delta_{0}^{\tau}}, \quad \tilde{C}_{\tau}^{\tau}=\frac{C_{0}^{\tau}}{\delta_{0}^{\tau}}, \quad \tilde{C}_{\tau}^{0}=\frac{C_{0}^{0}}{\delta_{0}^{\tau}}, \quad \tilde{B}_{\tau}^{s}=\frac{B_{0}^{s}}{\varepsilon_{0}^{\tau}}, \quad \tilde{B}_{\tau}^{\tau}=\frac{B_{0}^{\tau}}{\varepsilon_{0}^{\tau}}, \quad \tilde{B}_{\tau}^{0}=\frac{B_{0}^{0}}{\varepsilon_{0}^{\tau}}, \\
& B_{i}^{s}=-\tilde{B}_{i}^{s}, \quad B_{0}^{s}=\tilde{B}_{0}^{s}, \quad B_{\tau}^{s}=\tilde{B}_{\tau}^{s}, \quad C_{s}^{i}=-\tilde{C}_{s}^{i}, \quad C_{0}^{i}=\tilde{C}_{0}^{i}, \quad C_{\tau}^{i}=\tilde{C}_{\tau}^{i} \text {, } \\
& B_{i}^{\tau}=-\frac{\tilde{B}_{i}^{0}}{\tilde{\delta}_{\tau}^{0}}, \quad B_{0}^{\tau}=\frac{\tilde{B}_{0}^{0}}{\tilde{\delta}_{\tau}^{0}}, \quad B_{\tau}^{\tau}=\frac{\tilde{B}_{\tau}^{0}}{\tilde{\delta}_{\tau}^{0}}, \quad C_{s}^{\tau}=-\frac{\tilde{C}_{s}^{0}}{\tilde{\varepsilon}_{\tau}^{0}}, \quad C_{0}^{\tau}=\frac{\tilde{C}_{0}^{0}}{\tilde{\varepsilon}_{\tau}^{0}}, \quad C_{\tau}^{\tau}=\frac{\tilde{C}_{\tau}^{0}}{\tilde{\varepsilon}_{\tau}^{0}}, \\
& C_{0}^{i}=-\frac{\tilde{C}_{\tau}^{i}}{\tilde{\delta}_{\tau}^{0}}, \quad C_{0}^{0}=\frac{\tilde{C}_{\tau}^{0}}{\tilde{\delta}_{\tau}^{0}}, \quad C_{0}^{\tau}=\frac{\tilde{C}_{\tau}^{\tau}}{\tilde{\delta}_{\tau}^{0}}, \quad B_{0}^{s}=\frac{\tilde{B}_{\tau}^{s}}{\tilde{\varepsilon}_{\tau}^{0}}, \quad B_{0}^{0}=\frac{\tilde{B}_{\tau}^{0}}{\tilde{\varepsilon}_{\tau}^{0}}, \quad B_{0}^{\tau}=\frac{\tilde{B}_{\tau}^{\tau}}{\tilde{\varepsilon}_{\tau}^{0}},
\end{aligned}
$$


where

$$
\begin{aligned}
& \varepsilon_{J}^{I} \triangleq C_{S}^{I} B_{J}^{S}, \quad \delta_{T}^{S}=B_{I}^{S} C_{T}^{I}, \quad \varepsilon_{0}^{I} \triangleq B_{0}^{0} C_{0}^{I}=B_{0}^{S} C_{S}^{I}, \quad \delta_{0}^{S} \triangleq C_{0}^{0} B_{0}^{S}=C_{0}^{I} B_{I}^{S} . \\
& \tilde{\varepsilon}_{\nu}^{\mu} \triangleq \tilde{C}_{\alpha}^{\mu} \tilde{B}_{\nu}^{\alpha}, \quad \tilde{\delta}_{\beta}^{\alpha}=\tilde{B}_{\mu}^{\alpha} \tilde{C}_{\beta}^{\mu}, \quad \tilde{\varepsilon}_{\tau}^{\mu} \triangleq \tilde{B}_{\tau}^{\tau} \tilde{C}_{\tau}^{\mu}=\tilde{B}_{\tau}^{\alpha} \tilde{C}_{\alpha}^{\mu}, \quad \tilde{\delta}_{\tau}^{\alpha} \triangleq \tilde{C}_{\tau}^{\tau} \tilde{B}_{\tau}^{\alpha}=\tilde{C}_{\tau}^{\mu} \tilde{B}_{\mu}^{\alpha} .
\end{aligned}
$$

The evolution lemma of Proposition 3.3.2 can be expressed in Minkowski coordinate as

$$
\begin{array}{ll}
w^{\mu} \frac{\partial}{\partial \tilde{x}^{\mu}} \cong w^{\tau} \frac{d}{d \tilde{x}^{\tau}} \Leftrightarrow w^{\mu}=w^{\tau} \tilde{\varepsilon}_{\tau}^{\mu}, & \bar{w}_{\mu} \frac{\partial}{\partial \tilde{x}_{\mu}} \cong \bar{w}_{\tau} \frac{d}{d \tilde{x}_{\tau}} \Leftrightarrow \bar{w}_{\mu}=\bar{w}_{\tau} \tilde{\bar{\varepsilon}}_{\mu}^{\tau}, \\
w_{\mu} d \tilde{x}^{\mu} \simeq w_{\tau} d \tilde{x}^{\tau} \Leftrightarrow \tilde{\varepsilon}_{\tau}^{\mu} w_{\mu}=w_{\tau}, & \bar{w}^{\mu} d \tilde{x}_{\mu} \simeq \bar{w}^{\tau} d \tilde{x}_{\tau} \Leftrightarrow \tilde{\varepsilon}_{\mu}^{\tau} \bar{w}^{\mu}=\bar{w}^{\tau} .
\end{array}
$$

where

$$
\tilde{\bar{\varepsilon}}_{\nu}^{\mu} \triangleq \tilde{\bar{B}}_{\alpha}^{\mu} \tilde{\bar{C}}_{\nu}^{\alpha}=\varepsilon_{\nu}^{\mu}, \quad \tilde{\bar{\delta}}_{\beta}^{\alpha} \triangleq \tilde{\bar{C}}_{\mu}^{\alpha} \tilde{\bar{B}}_{\beta}^{\mu}=\delta_{\beta}^{\alpha}, \quad \tilde{\bar{\varepsilon}}_{\mu}^{\tau} \triangleq \tilde{\bar{B}}_{\tau}^{\tau} \tilde{\bar{C}}_{\mu}^{\tau}=\tilde{\bar{B}}_{\alpha}^{\tau} \tilde{\bar{C}}_{\mu}^{\alpha}, \quad \tilde{\bar{\delta}}_{\alpha}^{\tau} \triangleq \tilde{\bar{C}}_{\tau}^{\tau} \tilde{\bar{B}}_{\alpha}^{\tau}=\tilde{\bar{C}}_{\mu}^{\tau} \tilde{\bar{B}}_{\alpha}^{\mu}
$$

Discussion 4.3.2. Define $\left(d \xi^{\tau}\right)^{2} \triangleq \sum_{a=r+1}^{\mathfrak{D}}\left(d \xi^{a}\right)^{2}$ and $\left(d x^{\tau}\right)^{2} \triangleq \sum_{m=r+1}^{\mathfrak{D}}\left(d x^{m}\right)^{2}$. According to Definition 3.1.1, time metrics $d \xi^{0}$ and $d x^{0}$ on $(\tilde{M}, f)$ satisfy

$$
\begin{aligned}
& \left(d \xi^{0}\right)^{2} \triangleq \sum_{s=1}^{r}\left(d \xi^{s}\right)^{2}+\left(d \xi^{\tau}\right)^{2}=\delta_{S T} d \xi^{S} d \xi^{T}=G_{I J} d x^{I} d x^{J}, \quad G_{I J} \triangleq \delta_{S T} B_{I}^{S} B_{J}^{T} \\
& \left(d x^{0}\right)^{2} \triangleq \sum_{i=1}^{r}\left(d x^{i}\right)^{2}+\left(d x^{\tau}\right)^{2}=\varepsilon_{I J} d x^{I} d x^{J}=H_{S T} d \xi^{S} d \xi^{T}, \quad H_{S T} \triangleq \varepsilon_{I J} C_{S}^{I} C_{T}^{J} .
\end{aligned}
$$

Thus, we obtain proper-time metrics $d \tilde{\xi}^{\tau}$ and $d \tilde{x}^{\tau}$ in Minkowski coordinates as below:

$$
\begin{aligned}
& \left(d \tilde{\xi}^{\tau}\right)^{2}=\left(d \xi^{0}\right)^{2}-\sum_{s=1}^{r}\left(d \xi^{s}\right)^{2}=\tilde{\delta}_{\alpha \beta} d \tilde{\xi}^{\alpha} d \tilde{\xi}^{\beta}=\tilde{G}_{\mu \nu} d \tilde{x}^{\mu} d \tilde{x}^{\nu}, \quad \tilde{G}_{\mu \nu} \triangleq \tilde{\delta}_{\alpha \beta} \tilde{B}_{\mu}^{\alpha} \tilde{B}_{\nu}^{\beta}, \\
& \left(d \tilde{x}^{\tau}\right)^{2}=\left(d x^{0}\right)^{2}-\sum_{i=1}^{r}\left(d x^{i}\right)^{2}=\tilde{\varepsilon}_{\mu \nu} d \tilde{x}^{\mu} d \tilde{x}^{\nu}=\tilde{H}_{\alpha \beta} d \tilde{\xi}^{\alpha} d \tilde{\xi}^{\beta}, \quad \tilde{H}_{\alpha \beta} \triangleq \tilde{\varepsilon}_{\mu \nu} \tilde{C}_{\alpha}^{\mu} \tilde{C}_{\beta}^{\nu} .
\end{aligned}
$$

It is easy to know there are relations between the regular metric $G_{I J}$ and the Minkowski metric $\tilde{G}_{\mu \nu}$ as below:

$$
\begin{aligned}
& G_{\tau \tau}=\frac{\tilde{G}_{\tau \tau}}{\tilde{G}_{00}} G_{00}=\frac{\tilde{\delta}_{\tau}^{0} \tilde{\delta}_{\tau}^{0}}{\tilde{\varepsilon}_{\tau}^{0} \tilde{\varepsilon}_{\tau}^{0}} \frac{\tilde{G}_{\tau \tau}}{\tilde{G}_{00}} \tilde{G}_{\tau \tau}, \quad \quad \tilde{G}_{00}=\frac{G_{00}}{G_{\tau \tau}} \tilde{G}_{\tau \tau}=\frac{\delta_{0}^{\tau} \delta_{0}^{\tau}}{\varepsilon_{0}^{\tau} \varepsilon_{0}^{\tau}} \frac{G_{00}}{G_{\tau \tau}} G_{00}, \\
& G_{i \tau}=-\frac{\tilde{G}_{i 0}}{\tilde{G}_{00}} \tilde{\varepsilon}_{\tau}^{0} G_{00}=-\frac{\tilde{\delta}_{\tau}^{0} \tilde{\delta}_{\tau}^{0}}{\tilde{\varepsilon}_{\tau}^{0}} \frac{\tilde{G}_{\tau \tau}}{\tilde{G}_{00}} \tilde{G}_{i 0}, \quad \tilde{G}_{i 0}=-\frac{G_{i \tau}}{G_{\tau \tau}} \varepsilon_{0}^{\tau} \tilde{G}_{\tau \tau}=-\frac{\delta_{0}^{\tau} \delta_{0}^{\tau}}{\varepsilon_{0}^{\tau}} \frac{G_{00}}{G_{\tau \tau}} G_{i \tau}, \\
& G_{\tau j}=-\frac{\tilde{G}_{0 j}}{\tilde{G}_{00}} \tilde{\varepsilon}_{\tau}^{0} G_{00}=-\frac{\tilde{\delta}_{\tau}^{0} \tilde{\delta}_{\tau}^{0}}{\tilde{\varepsilon}_{\tau}^{0}} \frac{\tilde{G}_{\tau \tau}}{\tilde{G}_{00}} \tilde{G}_{0 j}, \quad \tilde{G}_{0 j}=-\frac{G_{\tau j}}{G_{\tau \tau}} \varepsilon_{0}^{\tau} \tilde{G}_{\tau \tau}=-\frac{\delta_{0}^{\tau}}{\varepsilon_{0}^{\tau}} \frac{\delta_{00}^{\tau}}{G_{\tau \tau}} G_{\tau j} \\
& G_{i j}=-\frac{\tilde{G}_{i j}}{\tilde{G}_{00}} G_{00}=-\frac{\tilde{\delta}_{\tau}^{0} \tilde{\delta}_{\tau}^{0}}{\tilde{\varepsilon}_{\tau}^{0} \tilde{\varepsilon}_{\tau}^{0}} \frac{\tilde{G}_{\tau \tau}}{\tilde{G}_{00}} \tilde{G}_{i j}, \quad \tilde{G}_{i j}=-\frac{G_{i j}}{G_{\tau \tau}} \tilde{G}_{\tau \tau}=-\frac{\delta_{0}^{\tau} \delta_{0}^{\tau}}{\varepsilon_{0}^{\tau} \varepsilon_{0}^{\tau}} \frac{G_{00}}{G_{\tau \tau}} G_{i j} .
\end{aligned}
$$

Denote $\tilde{G}_{\tau \tau} \triangleq \tilde{B}_{\tau}^{\tau} \tilde{B}_{\tau}^{\tau}, \tilde{G}^{\tau \tau} \triangleq \tilde{C}_{\tau}^{\tau} \tilde{C}_{\tau}^{\tau}$, then it is easy to obtain $\tilde{G}_{\tau \tau}=\frac{\delta_{0}^{\tau} \delta_{0}^{\tau}}{\varepsilon_{0}^{\tau} \varepsilon_{0}^{\tau}} G_{00}, \quad G_{00}=\frac{\tilde{\delta}_{\tau}^{0} \tilde{\delta}_{\tau}^{0}}{\tilde{\varepsilon}_{\tau}^{0} \tilde{\varepsilon}_{\tau}^{0}} \tilde{G}_{\tau \tau}$. 


\subsection{Affine connection representation of classical spacetime evolution}

Let $\tilde{D}$ be the simple connection on $\tilde{M}$, and denote $\tilde{t}_{L ; \tau} \triangleq \tilde{t}_{; \sigma} \tilde{\varepsilon}_{\tau}^{\sigma}$, then the absolute differential and gradient of section 3.4 can be expressed on $\tilde{M}$ in Minkowski coordinate as

$$
\begin{gathered}
\tilde{D} \tilde{t} \triangleq \tilde{t}_{; \sigma} d \tilde{x}^{\sigma}, \quad \tilde{D}_{L} \tilde{t}_{L} \triangleq \tilde{t}_{L ; \tau} d \tilde{x}^{\tau}, \\
\tilde{\nabla} \tilde{t} \triangleq \tilde{t}_{; \sigma} \frac{\partial}{\partial \tilde{x}_{\sigma}}, \quad \tilde{\nabla}_{L} \tilde{t}_{L} \triangleq \tilde{t}_{L ; \tau} \frac{d}{d \tilde{x}_{\tau}} .
\end{gathered}
$$

Evidently, $\tilde{D} \tilde{t} \simeq \tilde{D}_{L} \tilde{t}_{L}$ if and only if $L$ is an arbitrary path. $\tilde{\nabla} \tilde{t} \cong \tilde{\nabla}_{L} \tilde{t}_{L}$ if and only if $L$ is the gradient line.

Definition 4.4.1. Similar to section 3.6, suppose a charge $\tilde{\rho}$ of $\tilde{f}$ evolves on $(\tilde{M}, \tilde{g})$. We have the following definitions.

(1) $\tilde{m}^{\tau} \triangleq \tilde{\rho}^{; \tau}$ and $\tilde{m}_{\tau} \triangleq \tilde{\rho}_{; \tau}$ are said to be the rest mass of $\tilde{\rho}$.

(2) $\tilde{p}^{\mu} \triangleq-\tilde{\rho}^{; \mu}$ and $\tilde{p}_{\mu} \triangleq-\tilde{\rho}_{; \mu}$ are said to be the energy-momentum of $\tilde{\rho}$, and $\tilde{E}^{0} \triangleq \tilde{\rho}^{; 0}$, $\tilde{E}_{0} \triangleq \tilde{\rho}_{; 0}$ are said to be the energy of $\tilde{\rho}$.

(3) $\tilde{M}^{\tau} \triangleq \frac{d \tilde{\rho}}{d \tilde{x}_{\tau}}$ and $\tilde{M}_{\tau} \triangleq \frac{d \tilde{\rho}}{d \tilde{x}^{\tau}}$ are said to be the canonical rest mass of $\tilde{\rho}$.

(4) $\tilde{P}^{\mu} \triangleq-\frac{\partial \tilde{\rho}}{\partial \tilde{x}_{\mu}}$ and $\tilde{P}_{\mu} \triangleq-\frac{\partial \tilde{\rho}}{\partial \tilde{x}^{\mu}}$ are said to be the canonical energy-momentum of $\tilde{\rho}$, and $\tilde{H}^{0} \triangleq \frac{\partial \tilde{\rho}}{\partial \tilde{x}_{0}}, \tilde{H}_{0} \triangleq \frac{\partial \tilde{\rho}}{\partial \tilde{x}^{0}}$ are said to be the canonical energy of $\tilde{\rho}$.

Discussion 4.4.1. Similar to Proposition 3.6.1, $\forall p \in \tilde{M}$, if and only if the evolution direction $\left[L_{p}\right]=\left.\tilde{\nabla} \tilde{\rho}\right|_{p}$, the directional derivative is

$$
\left\langle\tilde{m}_{\tau} \frac{d}{d \tilde{x}_{\tau}}, \tilde{m}_{\tau} d \tilde{x}^{\tau}\right\rangle=\left\langle\tilde{p}_{\mu} \frac{\partial}{\partial \tilde{x}_{\mu}}, \tilde{p}_{\mu} d \tilde{x}^{\mu}\right\rangle,
$$

that is $\tilde{G}^{\tau \tau} \tilde{m}_{\tau} \tilde{m}_{\tau}=\tilde{G}^{\mu \nu} \tilde{p}_{\mu} \tilde{p}_{\nu}$, or

$$
\tilde{m}_{\tau} \tilde{m}^{\tau}=\tilde{p}_{\mu} \tilde{p}^{\mu},
$$

which is the affine connection representation of energy-momentum equation.

In addition, similar to Proposition 3.6.2, according to the evolution lemma, $\forall p \in \tilde{M}$, if and only if the evolution direction $\left[L_{p}\right]=\left.\tilde{\nabla} \tilde{\rho}\right|_{p}$, we have $\tilde{p}_{\mu}=-\tilde{m}_{\tau} \frac{d \tilde{x}_{\mu}}{d \tilde{x}_{\tau}}$, that is $\tilde{E}_{0}=\tilde{m}_{\tau} \frac{d \tilde{x}_{0}}{d \tilde{x}_{\tau}}=\tilde{m}_{\tau} \frac{d x_{0}}{d x_{\tau}}$ and $\tilde{p}_{i}=-\tilde{m}_{\tau} \frac{d \tilde{x}_{i}}{d \tilde{x}_{\tau}}=\tilde{m}_{\tau} \frac{-d \tilde{x}_{i}}{d \tilde{x}_{\tau}}=\tilde{m}_{\tau} \frac{d x_{i}}{d x_{\tau}}=\tilde{E}_{0} \frac{d x_{i}}{d x_{0}}$. This can also be regarded as the origin of $p=m v$.

Similar to discussions of section 3.7, denote

$$
\begin{array}{ll}
{\left[\tilde{\rho} \tilde{\Gamma}_{\omega}\right] \triangleq\left[\tilde{\rho}_{\mu \nu} \tilde{\Gamma}_{\omega}\right] \triangleq \frac{\partial \tilde{\rho}}{\partial \tilde{x}^{\omega}}-\tilde{\rho}_{j \omega} \triangleq \frac{\partial \tilde{\rho}_{\mu \nu}}{\partial \tilde{x}^{\omega}}-\tilde{\rho}_{\mu \nu ; \omega}=\tilde{\rho}_{\mu \chi} \tilde{\Gamma}_{\nu \omega}^{\chi}+\tilde{\rho}_{\chi \nu} \tilde{\Gamma}_{\mu \omega}^{\chi}, \quad\left[\tilde{\rho} \tilde{\Gamma}^{\omega}\right] \triangleq \tilde{G}^{\chi \omega}\left[\tilde{\rho} \tilde{\Gamma}_{\chi}\right],} \\
{\left[\tilde{\rho} \tilde{\Gamma}_{\tau}\right] \triangleq\left[\tilde{\rho}_{\mu \nu} \tilde{\Gamma}_{\tau}\right] \triangleq \frac{d \tilde{\rho}}{d \tilde{x}^{\tau}}-\tilde{\rho}_{; \tau} \triangleq \frac{d \tilde{\rho}_{\mu \nu}}{d \tilde{x}^{\tau}}-\tilde{\rho}_{\mu \nu ; \tau}=\tilde{\rho}_{\mu \chi} \tilde{\Gamma}_{\nu \tau}^{\chi}+\tilde{\rho}_{\chi \nu} \tilde{\Gamma}_{\mu \tau}^{\chi}, \quad\left[\tilde{\rho} \tilde{\Gamma}^{\tau}\right] \triangleq \tilde{G}^{\tau \tau}\left[\tilde{\rho} \tilde{\Gamma}_{\tau}\right] .} \\
{\left[\tilde{\rho} \tilde{R}_{\rho \sigma}\right] \triangleq \tilde{\rho}_{\mu \chi} \tilde{R}_{\nu \rho \sigma}^{\chi}+\tilde{\rho}_{\chi \nu} \tilde{R}_{\mu \rho \sigma}^{\chi},} \\
{\left[\tilde{\rho} \tilde{B}_{\rho \sigma}\right] \triangleq \tilde{\rho}_{\mu \chi}\left(\frac{\partial \tilde{\Gamma}_{\nu \sigma}^{\chi}}{\partial \tilde{x}^{\rho}}-\frac{\partial \tilde{\Gamma}_{\nu \rho}^{\chi}}{\partial \tilde{x}^{\sigma}}\right)+\tilde{\rho}_{\chi \nu}\left(\frac{\partial \tilde{\Gamma}_{\mu \sigma}^{\chi}}{\partial \tilde{x}^{\rho}}-\frac{\partial \tilde{\Gamma}_{\mu \rho}^{\chi}}{\partial \tilde{x}^{\sigma}}\right), \quad\left[\tilde{\rho} \tilde{E}_{\rho \sigma}\right] \triangleq\left[\tilde{\rho} \tilde{\Gamma}_{\sigma}\right]_{; \rho}-\left[\tilde{\rho} \tilde{\Gamma}_{\rho}\right]_{; \sigma},}
\end{array}
$$

Then for the same reason as section 3.7, based on Definition 4.4.1, we can strictly prove the affine connection representation of general Lorentz force equation of $\tilde{\rho}$, which is

$$
\tilde{f}_{\rho} \triangleq \tilde{p}_{\rho ; \tau}=\tilde{m}_{\tau ; \rho}-\tilde{p}_{\sigma} \tilde{\varepsilon}_{\tau ; \rho}^{\sigma}+\left[\tilde{\rho} \tilde{R}_{\rho \sigma}\right] \tilde{\varepsilon}_{\tau}^{\sigma} \text {. }
$$


And similarly, we also have $\tilde{T}_{\mu \nu}^{; \mu}=0$. In the mass-point model, $\tilde{m}_{\tau ; \rho}$ and $\tilde{\varepsilon}_{\tau ; \rho}^{\sigma}$ do not make sense, so Eq.(47) turns into

$$
\tilde{f}_{\rho}=\left[\tilde{\rho} \tilde{R}_{\rho \sigma}\right] \tilde{\varepsilon}_{\tau}^{\sigma} .
$$

This is the affine connection representation of the force of interaction (e.g. the Lorentz force $\boldsymbol{f}=q(\boldsymbol{E}+\boldsymbol{v} \times \boldsymbol{B})$ or $f_{\rho}=j^{\sigma} F_{\rho \sigma}$ of the electrodynamics $)$.

Definition 4.4.2. It is similar to Definition 3.6.2. Let $\tilde{\mathcal{P}}(b, a)$ be the totality of paths on $\tilde{M}$ from point $a$ to point $b$. And let $L \in \tilde{\mathcal{P}}(b, a)$, and parameter $\tilde{x}^{\tau}$ satisfy $\tau_{a} \triangleq \tilde{x}^{\tau}(a)<\tilde{x}^{\tau}(b) \triangleq \tau_{b}$. The affine connection representation of action in Minkowski coordinates can be defined as

$$
\tilde{\mathfrak{s}}(L) \triangleq \int_{L} \tilde{D} \tilde{\rho}=\int_{L} \tilde{p}_{\mu} d \tilde{x}^{\mu}=\int_{\tau_{a}}^{\tau_{b}} \tilde{m}_{\tau} d \tilde{x}^{\tau}, \quad \tilde{s}(L) \triangleq \int_{\tau_{a}}^{\tau_{b}}\left(\gamma^{\mu} \tilde{\rho}_{; \mu}+\tilde{m}_{\tau}\right) d \tilde{x}^{\tau} .
$$

There are more illustrations in Remark 4.5.1.

\subsection{Affine connection representation of Dirac equation}

Discussion 4.5.1. Define Dirac algebras $\gamma^{\mu}$ and $\gamma^{\alpha}$ such that

$$
\gamma^{\mu}=\tilde{C}_{\alpha}^{\mu} \gamma^{\alpha}, \quad \gamma^{\alpha} \gamma^{\beta}+\gamma^{\beta} \gamma^{\alpha}=2 \tilde{\delta}^{\alpha \beta}, \quad \gamma^{\mu} \gamma^{\nu}+\gamma^{\nu} \gamma^{\mu}=2 \tilde{G}^{\mu \nu}
$$

Suppose $(\tilde{M}, \tilde{g})$ is orthogonal. According to Definition 4.2.2 and Discussion 4.3.2, $\tilde{G}_{\tau \tau}=1$. Due to Discussion 4.4.1, in a gradient direction of $\tilde{\rho} \triangleq \tilde{\rho}_{\omega \nu}$, we have

$$
\begin{aligned}
\tilde{\rho}_{; \mu} \tilde{\rho}^{; \mu}=\tilde{\rho}_{; \tau} \tilde{\rho}^{; \tau} & \Leftrightarrow \tilde{G}^{\mu \nu} \tilde{\rho}_{; \mu} \tilde{\rho}_{; \nu}=\tilde{m}_{\tau}^{2} \\
& \Leftrightarrow\left(\gamma^{\mu} \tilde{\rho}_{; \mu}\right)\left(\gamma^{\nu} \tilde{\rho}_{; \nu}\right)+\left(\gamma^{\nu} \tilde{\rho}_{; \nu}\right)\left(\gamma^{\mu} \tilde{\rho}_{; \mu}\right)=2 \tilde{m}_{\tau}^{2} \\
& \Leftrightarrow\left(\gamma^{\mu} \tilde{\rho}_{; \mu}\right)\left(\gamma^{\nu} \tilde{\rho}_{; \nu}\right)=\tilde{m}_{\tau}^{2} \\
& \Leftrightarrow\left(\gamma^{\mu} \tilde{\rho}_{; \mu}\right)^{2}=\tilde{m}_{\tau}^{2} .
\end{aligned}
$$

Without loss of generality, take $\gamma^{\mu} \tilde{\rho}_{; \mu}=\tilde{m}_{\tau}$, that is

$$
\gamma^{\mu} \tilde{\rho}_{\omega \nu ; \mu}=\tilde{m}_{\omega \nu \tau}
$$

Next, denote

$$
\left[g \tilde{\Gamma}_{\mu}\right]^{\omega \nu} \triangleq \sum_{\sigma} \tilde{G}^{\nu \nu^{\prime}} \tilde{\Gamma}_{\nu^{\prime} \sigma \mu}+\sum_{\kappa} \tilde{G}^{\omega \omega^{\prime}} \tilde{\Gamma}_{\omega^{\prime} \kappa \mu}, \quad \tilde{D}_{\mu}^{\omega \nu} \triangleq \partial_{\mu}-\left[g \tilde{\Gamma}_{\mu}\right]^{\omega \nu} .
$$

From Eq.(49), it is obtained that

$$
\begin{aligned}
\sum_{\omega, \nu} \gamma^{\mu} \tilde{\rho}_{\omega \nu ; \mu}=\sum_{\omega, \nu} \tilde{m}_{\omega \nu \tau} & \Leftrightarrow \sum_{\omega, \nu} \gamma^{\mu}\left(\partial_{\mu} \rho_{\omega \nu}-\tilde{\rho}_{\omega \chi} \tilde{\Gamma}_{\nu \mu}^{\chi}-\tilde{\rho}_{\chi \nu} \tilde{\Gamma}_{\omega \mu}^{\chi}\right)=\sum_{\omega, \nu} \tilde{m}_{\omega \nu \tau} \\
& \Leftrightarrow \sum_{\omega, \nu} \gamma^{\mu}\left(\partial_{\mu} \rho_{\omega \nu}-\tilde{\rho}_{\omega \nu} \sum_{\sigma} \tilde{\Gamma}_{\sigma \mu}^{\nu}-\tilde{\rho}_{\omega \nu} \sum_{\kappa} \tilde{\Gamma}_{\kappa \mu}^{\omega}\right)=\sum_{\omega, \nu} \tilde{m}_{\omega \nu \tau} \\
& \Leftrightarrow \sum_{\omega, \nu} \gamma^{\mu}\left(\partial_{\mu} \rho_{\omega \nu}-\tilde{\rho}_{\omega \nu}\left[g \tilde{\Gamma}_{\mu}\right]^{\omega \nu}\right)=\sum_{\omega, \nu} \tilde{m}_{\omega \nu \tau} \\
& \Leftrightarrow \sum_{\omega, \nu} \gamma^{\mu}\left(\partial_{\mu}-\left[g \tilde{\Gamma}_{\mu}\right]^{\omega \nu}\right) \tilde{\rho}_{\omega \nu}=\sum_{\omega, \nu} \tilde{m}_{\omega \nu \tau},
\end{aligned}
$$


that is

$$
\sum_{\omega, \nu} \gamma^{\mu} \tilde{D}_{\mu}^{\omega \nu} \tilde{\rho}_{\omega \nu}=\sum_{\omega, \nu} \tilde{m}_{\omega \nu \tau}, \quad \tilde{D}_{\mu}^{\omega \nu} \triangleq \partial_{\mu}-\left[g \tilde{\Gamma}_{\mu}\right]^{\omega \nu}
$$

denoted concisely by

$$
\gamma^{\mu} \tilde{D}_{\mu} \tilde{\rho}=\tilde{m}_{\tau}, \quad \tilde{D}_{\mu} \triangleq \partial_{\mu}-\left[g \tilde{\Gamma}_{\mu}\right] .
$$

We speak of Eq.(49) and (51) as affine Dirac equations.

Discussion 4.5.2. Next, we construct a kind of complex-valued representation of affine Dirac equation. The restriction of the charge $\tilde{\rho}_{\omega \nu}$ to $\left(\tilde{U}, \tilde{x}^{\mu}\right)$ is a function $\tilde{\rho}_{\omega \nu}\left(\tilde{x}^{\mu}\right)$ with respect to the coordinates $\left(\tilde{x}^{\mu}\right) \triangleq\left(\tilde{x}^{0}, \tilde{x}^{1}, \tilde{x}^{2}, \tilde{x}^{3}\right)$. Let

$$
\tilde{\mathrm{P}}_{\omega \nu}\left(\tilde{x}^{0}\right) \triangleq \int_{\left(\tilde{x}^{1}, \tilde{x}^{2}, \tilde{x}^{3}\right)} \tilde{\rho}_{\omega \nu}\left(\tilde{x}^{\mu}\right) d^{3} \tilde{x} .
$$

Suppose a function $f_{\omega \nu}=f_{\omega \nu}\left(\tilde{x}^{\mu}\right)$ on $\left(\tilde{U}, \tilde{x}^{\mu}\right)$ satisfies that

$$
\tilde{\rho}_{\omega \nu}=\left(f_{\omega \nu}\right)^{2} \tilde{\mathrm{P}}_{\omega \nu}, \quad \int_{\left(\tilde{x}^{1}, \tilde{x}^{2}, \tilde{x}^{3}\right)}\left(f_{\omega \nu}\right)^{2} d^{3} \tilde{x}=1, \quad \varepsilon_{\tau}^{\mu} \frac{\partial f_{\omega \nu}}{\partial \tilde{x}^{\mu}}=0, \quad \gamma^{\mu} \frac{\partial f_{\omega \nu}}{\partial \tilde{x}^{\mu}}=0 .
$$

We define $\psi_{\omega \nu}$ and $\tilde{\mathbb{M}}_{\omega \nu \tau}$ in the following way.

$\tilde{y}_{\omega \nu} \triangleq \int_{L} d \tilde{\rho}_{\omega \nu}=\int_{L} \frac{d \tilde{\rho}_{\omega \nu}}{d \tilde{x}^{\tau}} d \tilde{x}^{\tau}=\int_{L}\left(\frac{d\left(f_{\omega \nu}^{2}\right)}{d \tilde{x}^{\tau}} \tilde{\mathrm{P}}_{\omega \nu}+f_{\omega \nu}^{2} \frac{d \tilde{\mathrm{P}}_{\omega \nu}}{d \tilde{x}^{\tau}}\right) d \tilde{x}^{\tau}=f_{\omega \nu}^{2} \int_{L} \frac{d \tilde{\mathrm{P}}_{\omega \nu}}{d \tilde{x}^{\tau}} d \tilde{x}^{\tau} \triangleq f_{\omega \nu}^{2} \tilde{Y}_{\omega \nu}$ $\psi_{\omega \nu} \triangleq f_{\omega \nu} e^{i \tilde{Y}_{\omega \nu}}, \quad \quad \tilde{m}_{\omega \nu \tau} \triangleq \tilde{\rho}_{\omega \nu ; \tau}=\left(f_{\omega \nu}^{2}\right)_{, \tau} \tilde{\mathrm{P}}_{\omega \nu}+f_{\omega \nu}^{2} \tilde{\mathrm{P}}_{\omega \nu ; \tau}=f_{\omega \nu}^{2} \tilde{\mathrm{P}}_{\omega \nu ; \tau} \triangleq f_{\omega \nu}^{2} \tilde{\mathbb{M}}_{\omega \nu \tau}$.

In the QFT propagator, we usually take $S$ in the path integral $\int e^{i S} \mathcal{D} \psi$ of a fermion in the form of

$$
-\int\left(i \bar{\psi} \gamma^{\mu} D_{\mu} \psi-\bar{\psi} \tilde{\mathbb{M}}_{\tau} \psi\right) d^{4} \tilde{x}
$$

where $S$ and $d^{4} \tilde{x}$ are both covariant. We believe that the external spatial integral $\int_{\left(\tilde{x}^{1}, \tilde{x}^{2}, \tilde{x}^{3}\right)} d^{3} \tilde{x}$ is not an essential part for evolution, so for the sake of simplicity, we do not take into account the external spatial part $\int_{\left(\tilde{x}^{1}, \tilde{x}^{2}, \tilde{x}^{3}\right)} d^{3} \tilde{x}$, but only consider the evolution part $\int_{L} d \tilde{x}^{0}$. Meanwhile, in order to remain the covariance, $\int_{L} d \tilde{x}^{0}$ has to be replaced by $\int_{L} d \tilde{x}^{\tau}$. Thus, in affine connection representation of gauge fields, we shall consider an action in the form of

$$
-\int_{L}\left(i \bar{\psi} \gamma^{\mu} D_{\mu} \psi-\bar{\psi} \tilde{\mathbb{M}}_{\tau} \psi\right) d \tilde{x}^{\tau}
$$

Concretely speaking, denote

$$
\tilde{D}_{\omega \nu \mu} \triangleq \frac{\partial}{\partial \tilde{x}^{\mu}}-i\left[\tilde{\mathrm{P}} \tilde{\Gamma}_{\mu}\right]_{\omega \nu}, \quad\left[\tilde{\mathrm{P}} \tilde{\Gamma}_{\mu}\right]_{\omega \nu} \triangleq \sum_{\sigma} \tilde{\mathrm{P}}_{\omega \nu} \tilde{\Gamma}_{\sigma \mu}^{\nu}+\sum_{\kappa} \tilde{\mathrm{P}}_{\omega \nu} \tilde{\Gamma}_{\kappa \mu}^{\omega} .
$$


From Eq.(48), we have

$$
\tilde{s}_{\omega \nu}(L) \triangleq \int_{L}\left(\gamma^{\mu} \tilde{\rho}_{\omega \nu ; \mu}+\tilde{m}_{\omega \nu \tau}\right) d \tilde{x}^{\tau}
$$

And from Eq.(50) we know $\sum_{\omega, \nu} \gamma^{\mu} \tilde{\rho}_{\omega \nu ; \mu}=\sum_{\omega, \nu} \gamma^{\mu} \tilde{D}_{\mu}^{\omega \nu} \tilde{\rho}_{\omega \nu}$. Then it is obtained that

$$
\begin{aligned}
& \tilde{s}_{\tilde{\rho}}(L) \triangleq \sum_{\omega, \nu} \tilde{s}_{\omega \nu}(L) \\
& =\int_{L} \sum_{\omega, \nu}\left(\gamma^{\mu} \tilde{\rho}_{\omega \nu ; \mu}+\tilde{m}_{\omega \nu \tau}\right) d \tilde{x}^{\tau} \\
& =\int_{L} \sum_{\omega, \nu}\left(\gamma^{\mu} \tilde{D}_{\mu}^{\omega \nu} \tilde{\rho}_{\omega \nu}+\tilde{m}_{\omega \nu \tau}\right) d \tilde{x}^{\tau} \\
& =\int_{L} \sum_{\omega, \nu}\left(\gamma^{\mu}\left(\partial_{\mu} \tilde{\rho}_{\omega \nu}-\left[g \tilde{\Gamma}_{\mu}\right]^{\omega \nu} \tilde{\rho}_{\omega \nu}\right)+\tilde{m}_{\omega \nu \tau}\right) d \tilde{x}^{\tau} \\
& =\int_{L} \sum_{\omega, \nu}\left(\gamma^{\mu}\left(\partial_{\mu} \tilde{y}_{\omega \nu}-\left[g \tilde{\Gamma}_{\mu}\right]^{\omega \nu} \tilde{\rho}_{\omega \nu}\right)+\tilde{m}_{\omega \nu \tau}\right) d \tilde{x}^{\tau} \\
& =\int_{L} \sum_{\omega, \nu}\left(\gamma^{\mu}\left(\partial_{\mu}\left(f_{\omega \nu}^{2} \tilde{Y}_{\omega \nu}\right)-\left[g \tilde{\Gamma}_{\mu}\right]^{\omega \nu} f_{\omega \nu}^{2} \tilde{\mathrm{P}}_{\omega \nu}\right)+f_{\omega \nu}^{2} \tilde{\mathbb{M}}_{\omega \nu \tau}\right) d \tilde{x}^{\tau} \\
& =\int_{L} \sum_{\omega, \nu}\left(\gamma^{\mu}\left(\partial_{\mu} \tilde{Y}_{\omega \nu}-\left[\tilde{\mathrm{P}} \tilde{\Gamma}_{\mu}\right]_{\omega \nu}\right) f_{\omega \nu}^{2}+f_{\omega \nu}^{2} \tilde{\mathbb{M}}_{\omega \nu \tau}\right) d \tilde{x}^{\tau} \\
& =\int_{L} \sum_{\omega, \nu}\left(f_{\omega \nu} e^{-i \tilde{Y}_{\omega \nu}} \gamma^{\mu}\left(f_{\omega \nu} e^{i \tilde{Y}_{\omega \nu}} \partial_{\mu} \tilde{Y}_{\omega \nu}-\left[\tilde{\mathrm{P}} \tilde{\Gamma}_{\mu}\right]_{\omega \nu} f_{\omega \nu} e^{i \tilde{Y}_{\omega \nu}}\right)+f_{\omega \nu} e^{-i \tilde{Y}_{\omega \nu}} \tilde{\mathbb{M}}_{\omega \nu \tau} f_{\omega \nu} e^{i \tilde{Y}_{\omega \nu}}\right) d \tilde{x}^{\tau} \\
& =\int_{L} \sum_{\omega, \nu}\left(-\bar{\psi}_{\omega \nu} i \gamma^{\mu}\left(e^{i \tilde{Y}_{\omega \nu}} \partial_{\mu} f_{\omega \nu}+f_{\omega \nu} e^{i \tilde{Y}_{\omega \nu}} i \partial_{\mu} \tilde{Y}_{\omega \nu}-i\left[\tilde{\mathrm{P}} \tilde{\Gamma}_{\mu}\right]_{\omega \nu} \psi_{\omega \nu}\right)+\bar{\psi}_{\omega \nu} \tilde{\mathbb{M}}_{\omega \nu \tau} \psi_{\omega \nu}\right) d \tilde{x}^{\tau} \\
& =\int_{L} \sum_{\omega, \nu}\left(-\bar{\psi}_{\omega \nu} i \gamma^{\mu}\left(\partial_{\mu}\left(f_{\omega \nu} e^{i \tilde{Y}_{\omega \nu}}\right)-i\left[\tilde{\mathrm{P}} \tilde{\Gamma}_{\mu}\right]_{\omega \nu} \psi_{\omega \nu}\right)+\bar{\psi}_{\omega \nu} \tilde{\mathbb{M}}_{\omega \nu \tau} \psi_{\omega \nu}\right) d \tilde{x}^{\tau} \\
& =\int_{L} \sum_{\omega, \nu}\left(-\bar{\psi}_{\omega \nu} i \gamma^{\mu}\left(\partial_{\mu}-i\left[\tilde{\mathrm{P}} \tilde{\Gamma}_{\mu}\right]_{\omega \nu}\right) \psi_{\omega \nu}+\bar{\psi}_{\omega \nu} \tilde{\mathbb{M}}_{\omega \nu \tau} \psi_{\omega \nu}\right) d \tilde{x}^{\tau} \\
& =\int_{L} \sum_{\omega, \nu}\left(-\bar{\psi}_{\omega \nu} i \gamma^{\mu} \tilde{D}_{\omega \nu \mu} \psi_{\omega \nu}+\bar{\psi}_{\omega \nu} \tilde{\mathbb{M}}_{\omega \nu \tau} \psi_{\omega \nu}\right) d \tilde{x}^{\tau}
\end{aligned}
$$

Hence,

$$
\delta \tilde{s}_{\tilde{\rho}}(L)=0 \Rightarrow i \gamma^{\mu} \tilde{D}_{\omega \nu \mu} \psi_{\omega \nu}=\tilde{\mathbb{M}}_{\omega \nu \tau} \psi_{\omega \nu} .
$$

Thus, we have obtained a complex-valued representation of gradient direction of $\tilde{\rho}_{\omega \nu}$. It can be denoted concisely by

$$
i \gamma^{\mu} \tilde{D}_{\mu} \psi=\tilde{\mathbb{M}}_{\tau} \psi
$$


Remark 4.5.1. From the above discussion, we know in the gradient direction of $\rho_{\omega \nu}$, that

$$
-\sum_{\omega, \nu} \bar{\psi}_{\omega \nu} i \gamma^{\mu} \tilde{D}_{\omega \nu \mu} \psi_{\omega \nu} d \tilde{x}^{\tau}=\sum_{\omega, \nu} \tilde{D} \tilde{\rho}_{\omega \nu}
$$

This shows that $s(L)$ and $\tilde{s}(L)$ in Definition 3.6.2 and Remark 3.6.1 are indeed applicable for constructing propagator by $e^{i s(L)}$ and $e^{i \tilde{s}(L)}$ in affine connection representation of gauge fields. Therefore, the idea in Discussion 3.10.3 are reasonable.

\subsection{From classical spacetime back to full-dimensional space}

Discussion 4.6.1. Now there is a problem. As is shown in $\operatorname{Remark} 4.2 .1,(\tilde{M}, \tilde{f})$ and $(\tilde{M}, \tilde{g})$ cannot totally reflect all the intrinsic geometric properties of internal space of $(M, f)$ and $(M, g)$. Concretely speaking:

In the previous section, we discuss the affine Dirac equation $\gamma^{\mu} \tilde{\rho}_{\omega \nu ; \mu}=\tilde{m}_{\omega \nu \tau}$ on $(\tilde{M}, \tilde{g})$. Similar to section 3.5, on $(\tilde{M}, \tilde{f})$ we have the affine Yang-Mills equation $\tilde{K}_{\nu \rho \sigma}^{\mu}{ }^{: \rho}=\tilde{\rho}_{\nu}^{\mu} \gamma_{\sigma}$ on $(\tilde{M}, \tilde{f})$. Suppose there is no gravitaional field, or to say, the external space is flat, then remaining non-vanish equations are just only

$$
\gamma^{\mu} \tilde{\rho}_{00 ; \mu}=\tilde{m}_{00 \tau}, \quad \tilde{K}_{0 \rho \sigma}^{0}{ }^{: \rho}=\tilde{\rho}_{0}^{0} \gamma_{\sigma}
$$

There are multiple internal charges

$$
\rho_{m n}(m, n=4,5, \cdots, \mathfrak{D})
$$

on $(M, f)$. We intend to use these $\rho_{m n}$ to describe leptons and hadrons. However, via encapsulation of classical spacetime, $(\tilde{M}, \tilde{f})$ remains only one internal charge $\tilde{\rho}_{00}$, it falls short. It is impossible for the only one real-valued field function $\tilde{\rho}_{00}$ to describe so many leptons and hadrons.

On the premise of not abandoning the $(1+3)$-dimensional spacetime, if we want to describe gauge fields, there is a method that to use some non-coordinate abstract degrees of freedom that is irrelevant to view of time and space on the phase $e^{i T_{a} \theta^{a}}$ of a complex-valued field function $\psi$, just like that in Standard Model. This way is effective, but not natural. It is not satisfying for a theory to adopt a coordinate representation for external space but a non-coordinate representation for internal space.

A logically more natural way is required to abandon the framework of $(1+3)$-dimensional spacetime $(\tilde{M}, \tilde{f})$ and $(\tilde{M}, \tilde{g})$. We should put internal space and external space together to describe their unified geometry with the same spatial frame. On $(M, f)$ and $(M, g)$, there are enough realvalued field functions $\rho_{m n}$ to describe leptons and hadrons, and enough internal components $\Gamma_{n P}^{m}$ of affine connection to describe gauge potentials.

Therefore, only on the full-dimensional $(M, f)$ and $(M, g)$ can total advantages of affine connection representation of gauge fields be brought into full play, and thereby show complete details of geometric properties of gauge field. So we are going to stop the discussions about the classical spacetime $\tilde{M}$, but to focus on the full-dimensional manifold $M$.

Discussion 4.6.2. On $M$, due to $\Gamma_{N P}^{M}=\frac{1}{2} C_{A}^{M}\left(\frac{\partial B_{N}^{A}}{\partial x^{P}}+\frac{\partial B_{P}^{A}}{\partial x^{N}}\right)$ and $G_{M N}=\delta_{A B} B_{M}^{A} B_{N}^{B}$ we know that gauge field and gravitational field can both be described by the unified spatial frames $B_{M}^{A}$ and $C_{A}^{M}$ of a reference-system $f$ or $g$. Reference-system is the common origination of gauge field and gravitational field. 
We adopt the components of $\Gamma_{N P}^{M}$ with $M, N \in\{4,5, \cdots, \mathfrak{D}\}$ to describe typical gauge fields such as electromagnetic, weak and strong interaction fields. The other components of $\Gamma_{N P}^{M}$ describe gravitational field. Simple connection $\Gamma_{N P}^{M}$ is the common affine connection representation of gauge field and gravitational field.

We adopt the components of $\rho_{M N}$ with $M, N \in\{4,5, \cdots, \mathfrak{D}\}$ to describe the charges of leptons and hadrons. The meanings of the other components of $\rho_{M N}$ are not clear at present, maybe they could be used to describe dark matter.

On orthogonal $(M, g)$ and $(M, f)$, there are full-dimensional field equations, i.e. affine Dirac equation and affine Yang-Mills equation

$$
\gamma^{P} \rho_{M N ; P}=\rho_{M N ; 0}, \quad K_{N P Q}^{M}: P=\rho_{N}^{M} \gamma_{Q}
$$

which reflect the on-shell evolution directions $\nabla \rho$ and $\nabla t$, respectively. Their quantum evolutions are described by the propagators in Definition 3.10.5 or Discussion 3.10.3.

Discussion 4.6.3. Gauge invariance and general covariance on $(M, g)$ are unified into invariance under the reference-system transformation $L_{k}: g \rightarrow g^{\prime}$.

On an orthogonal $(M, g)$, Eq.(52) presents as a full-dimensional action

$$
s_{\rho}(L)=\int_{L} \sum_{M, N}\left(\gamma^{P} \rho_{M N ; P}+\varepsilon_{0}^{P} \rho_{M N ; P}\right) d x^{0}=-i \int_{L} \sum_{M, N} \bar{\psi}_{M N}\left(\gamma^{P} D_{M N P}+\varepsilon_{0}^{P} D_{M N P}\right) \psi_{M N} d x^{0} .
$$

If and only if $L_{k}: g \rightarrow g^{\prime}$ is an orthogonal transformation, $L_{k}$ sends $s_{\rho}(L)$ to

$s_{\rho}^{\prime}(L)=\int_{L} \sum_{M, N}\left(\gamma^{P^{\prime}} \rho_{M N ; P^{\prime}}+\varepsilon_{0^{\prime}}^{P^{\prime}} \rho_{M N ; P^{\prime}}\right) d x^{0^{\prime}}=-i \int_{L} \sum_{M, N} \bar{\psi}_{M N}^{\prime}\left(\gamma^{P^{\prime}} D_{M N P^{\prime}}^{\prime}+\varepsilon_{0^{\prime}}^{P^{\prime}} D_{M N P^{\prime}}^{\prime}\right) \psi_{M N}^{\prime} d x^{0^{\prime}}$.

It is evident that

(i) Because $\rho_{M N}$ is determined by the reference-system $f$ but not $g, \rho_{M N}$ remains unchanged under the transformation $L_{k}: g \rightarrow g^{\prime}$.

(ii) $L_{k}$ makes $\rho_{M N ; P}, \psi_{M N}$ and $D_{M N P}$ changed, i.e.

$$
\begin{aligned}
L_{k}: \rho_{M N ; P} & \triangleq \frac{\partial \rho_{M N}}{\partial x^{P}}-\left(\rho_{M H}\left(\Gamma_{g}\right)_{N P}^{H}+\rho_{H N}\left(\Gamma_{g}\right)_{M P}^{H}\right) \\
& \mapsto \rho_{M N ; P^{\prime}}=\frac{\partial \rho_{M N}}{\partial x^{P^{\prime}}}-\left(\rho_{M H}\left(\Gamma_{g^{\prime}}\right)_{N P^{\prime}}^{H}+\rho_{H N}\left(\Gamma_{g^{\prime}}\right)_{M P^{\prime}}^{H}\right), \\
L_{k}: \psi_{M N} \triangleq & f_{M N} e^{i Y_{M N}}=f_{M N} \exp \left\{i \int_{L} \mathrm{P}_{M N ; 0} d x^{0}+\left(\mathrm{P}_{M H}\left(\Gamma_{g}\right)_{N P}^{H}+\mathrm{P}_{H N}\left(\Gamma_{g}\right)_{M P}^{H}\right) d x^{P}\right\} \\
& \mapsto \psi_{M N}^{\prime}=f_{M N} \exp \left\{i \int_{L} \mathrm{P}_{M N ; 0^{\prime}} d x^{0^{\prime}}+\left(\mathrm{P}_{M H}\left(\Gamma_{g^{\prime}}\right)_{N P^{\prime}}^{H}+\mathrm{P}_{H N}\left(\Gamma_{g^{\prime}}\right)_{M P^{\prime}}^{H}\right) d x^{P^{\prime}}\right\}, \\
L_{k}: D_{M N P} & \triangleq \frac{\partial}{\partial x^{P}}-i\left(\sum_{H} \mathrm{P}_{M N}\left(\Gamma_{g}\right)_{H P}^{N}+\sum_{G} \mathrm{P}_{M N}\left(\Gamma_{g}\right)_{G P}^{M}\right) \\
& \mapsto D_{M N P^{\prime}}^{\prime}=\frac{\partial}{\partial x^{P^{\prime}}}-i\left(\sum_{H} \mathrm{P}_{M N}\left(\Gamma_{g^{\prime}}\right)_{H P^{\prime}}^{N}+\sum_{G} \mathrm{P}_{M N}\left(\Gamma_{g^{\prime}}\right)_{G P^{\prime}}^{M}\right),
\end{aligned}
$$


where $\left(\Gamma_{g^{\prime}}\right)_{N P^{\prime}}^{M}=\left(B_{k}\right)_{M^{\prime}}^{M}\left(C_{k}\right)_{N}^{N^{\prime}}\left(\Gamma_{g^{\prime}}\right)_{N^{\prime} P^{\prime}}^{M^{\prime}}$, and $L_{k}:\left(\Gamma_{g}\right)_{N P}^{M} \mapsto\left(\Gamma_{g^{\prime}}\right)_{N^{\prime} P^{\prime}}^{M^{\prime}}$ transforms according to Proposition 2.5.1, that is

$$
L_{k}:\left(\Gamma_{g}\right)_{N P}^{M} \mapsto\left(\Gamma_{g^{\prime}}\right)_{N^{\prime} P^{\prime}}^{M^{\prime}}=\left(\Gamma_{g}\right)_{N P}^{M}\left(C_{k}\right)_{M}^{M^{\prime}}\left(B_{k}\right)_{N^{\prime}}^{N}\left(B_{k}\right)_{P^{\prime}}^{P}+\left(\Gamma_{k}\right)_{N^{\prime} P^{\prime}}^{M^{\prime}}
$$

Therefore, in affine connection representation of gauge fields, the gauge transformations $\psi \mapsto \psi^{\prime}$ and $D \mapsto D^{\prime}$ essentially boil down to the reference-system transformation $L_{k}$.

(iii) From the real-valued representation of action $s_{M N}(L)=\int_{L}\left(\gamma^{P} \rho_{M N ; P}+\varepsilon_{0}^{P} \rho_{M N ; P}\right) d x^{0}$, it can be seen evidently that

$$
L_{k}: s_{\rho}(L) \mapsto s_{\rho}^{\prime}(L)=s_{\rho}(L) .
$$

Remark 1. The above (ii) and (iii) show the unified gauge invariance and general covariance under the reference-system transformation $L_{k}$.

Remark 2. For a general $(M, g), g$ is not necessarily orthogonal, so the corresponding action should be described by

$$
s_{M N}(L)=\int_{L}\left(B_{0}^{0} \gamma^{P} \rho_{M N ; P}+\varepsilon_{0}^{P} \rho_{M N ; P}\right) d x^{0} .
$$

In this general case, Definition 3.6.2 and the method in Discussion 3.10.3 are also available and effective, where we take

$$
s_{M N}(L)=\int_{L} D \rho_{M N}
$$

Remark 3. We see that the real-valued representation of action is more concise than the complexvalued representation of action. Hence, it is more convenient to adopt real-valued representations for field function, field equation and action.

The research objects in the following sections are based on the following definition. We are going to use the simple connection $\Gamma_{N P}^{M}$ to show the affine connection representations of electromagnetic, weak and strong interaction fields, and to adopt the real-valued representation $\rho_{M N ; P}$ to discuss the interactions between gauge fields and elementary particles.

Definition 4.6.1. Let there be a geometric manifold $(M, f)$, such that $M=P \times N, r \triangleq \operatorname{dim} P=3$ and $\mathfrak{D} \triangleq \operatorname{dim} M=5$ or 6 or 8 .

(1) $\forall p \in M$, suppose the coordinate representation of $f(p)$ takes the form of

$$
\xi^{a}=\xi^{a}\left(x^{m}\right), \quad \xi^{s}=\delta_{i}^{s} x^{i}, \quad(s, i=1,2,3 ; a, m=4,5, \cdots, \mathfrak{D}),
$$

and $f$ satisfies the internal standard conditions that

$$
\text { (i) } G_{m n}=\text { const, } \quad \text { (ii) when } m \neq n, G_{m n}=0 \text {. }
$$

We say $f$ is a typical gauge field, and $L_{[f]}$ is a typical gauge transformation.

(2) $\forall p \in M$, suppose the coordinate representation of $f(p)$ takes the form of

$$
\xi^{a}=\xi^{a}\left(x^{M}\right), \quad \xi^{s}=\xi^{s}\left(x^{i}\right), \quad(s, i=1,2,3 ; a=4,5, \cdots, \mathfrak{D} ; M=1,2, \cdots, \mathfrak{D}) .
$$

We say $f$ is a typical gauge field with gravitation, and $L_{f}$ is a gravitational gauge transformation. 


\section{Affine connection representation of the gauge field of weak-electromagnetic interaction}

Definition 5.1. Suppose a geometric manifold $(M, f)$ satisfies $\mathfrak{D}=r+2=5$. $\forall p \in M$, the coordinate representation of $f(p)$ takes the form of

$$
\xi^{a}=\xi^{a}\left(x^{m}\right), \quad \xi^{s}=\delta_{i}^{s} x^{i}, \quad(s, i=1,2,3 ; a, m=4,5),
$$

and $f$ satisfies the internal standard conditions (55) of Definition 4.6.1(1) and

$$
G^{(\mathfrak{D}-1)(\mathfrak{D}-1)}=G^{\mathfrak{D} \mathfrak{D}} .
$$

We say $f$ is a weak and electromagnetic unified field. The reason for such naming lies in the following proposition.

Proposition 5.1. Let the simple connection of the above $(M, f)$ be $\Lambda_{N P}^{M}$ and $\Lambda_{M N P}$. And let the coefficients of curvature of $(M, f)$ be $K_{N P Q}^{M}$ and $K_{M N P Q}$. Denote

$$
\begin{aligned}
& \left\{\begin{array}{l}
B_{P} \triangleq \frac{1}{\sqrt{2}}\left(\Lambda_{\mathfrak{D} \mathfrak{D} P}+\Lambda_{(\mathfrak{D}-1)(\mathfrak{D}-1) P}\right) \\
A_{P}^{3} \triangleq \frac{1}{\sqrt{2}}\left(\Lambda_{\mathfrak{D} \mathfrak{D} P}-\Lambda_{(\mathfrak{D}-1)(\mathfrak{D}-1) P}\right)
\end{array}, \quad\left\{\begin{array}{l}
A_{P}^{1} \triangleq \frac{1}{\sqrt{2}}\left(\Lambda_{(\mathfrak{D}-1) \mathfrak{D} P}+\Lambda_{\mathfrak{D}(\mathfrak{D}-1) P}\right) \\
A_{P}^{2} \triangleq \frac{1}{\sqrt{2}}\left(\Lambda_{(\mathfrak{D}-1) \mathfrak{D} P}-\Lambda_{\mathfrak{D}(\mathfrak{D}-1) P}\right)
\end{array}\right.\right. \\
& \left\{\begin{array}{l}
B_{P Q} \triangleq \frac{1}{\sqrt{2}}\left(K_{\mathfrak{D} \mathfrak{D} P Q}+K_{(\mathfrak{D}-1)(\mathfrak{D}-1) P Q}\right) \\
F_{P Q}^{3} \triangleq \frac{1}{\sqrt{2}}\left(K_{\mathfrak{D} \mathfrak{D} P Q}-K_{(\mathfrak{D}-1)(\mathfrak{D}-1) P Q}\right)
\end{array}, \quad\left\{\begin{array}{l}
F_{P Q}^{1} \triangleq \frac{1}{\sqrt{2}}\left(K_{(\mathfrak{D}-1) \mathfrak{D} P Q}+K_{\mathfrak{D}(\mathfrak{D}-1) P Q}\right) \\
F_{P Q}^{2} \triangleq \frac{1}{\sqrt{2}}\left(K_{(\mathfrak{D}-1) \mathfrak{D} P Q}-K_{\mathfrak{D}(\mathfrak{D}-1) P Q}\right)
\end{array} .\right.\right.
\end{aligned}
$$

And denote $g \triangleq \sqrt{\left(G^{(\mathfrak{D}-1)(\mathfrak{D}-1)}\right)^{2}+\left(G^{\mathfrak{D} \mathfrak{D}}\right)^{2}}$. Thus the following equations hold, and they are all intrinsic geometric properties of $(M, f)$.

$$
\left\{\begin{array}{l}
B_{P Q}=\frac{\partial B_{Q}}{\partial x^{P}}-\frac{\partial B_{P}}{\partial x^{Q}} \\
F_{P Q}^{3}=\frac{\partial A_{Q}^{3}}{\partial x^{P}}-\frac{\partial A_{P}^{3}}{\partial x^{Q}}+g\left(A_{P}^{1} A_{Q}^{2}-A_{P}^{2} A_{Q}^{1}\right) \\
F_{P Q}^{1}=\frac{\partial A_{Q}^{1}}{\partial x^{P}}-\frac{\partial A_{P}^{1}}{\partial x^{Q}}+g\left(A_{P}^{2} A_{Q}^{3}-A_{P}^{3} A_{Q}^{2}\right) \\
F_{P Q}^{2}=\frac{\partial A_{Q}^{2}}{\partial x^{P}}-\frac{\partial A_{P}^{2}}{\partial x^{Q}}-g\left(A_{P}^{3} A_{Q}^{1}-A_{P}^{1} A_{Q}^{3}\right)
\end{array}\right.
$$

Proof. According to the definition, the semi-metric of $f$ satisfies that

$$
B_{m}^{s}=0, C_{a}^{i}=0 . B_{i}^{s}=\delta_{i}^{s}, \quad B_{i}^{a}=0, C_{s}^{i}=\delta_{s}^{i}, C_{s}^{m}=0 .
$$

The metric of $f$ satisfies that

$$
G_{m n}=0(m \neq n), G_{m n}=\text { const }, G_{M N} \triangleq \delta_{A B} B_{M}^{A} B_{N}^{B}, G^{M N}=\delta^{A B} C_{A}^{M} C_{B}^{N} .
$$




\section{Concretely:}

$$
\left\{\begin{array}{l}
G_{i j}=\delta_{s t} B_{i}^{s} B_{j}^{t}+\delta_{a b} B_{i}^{a} B_{j}^{b}=\delta_{s t} \delta_{i}^{s} \delta_{j}^{t}=\delta_{i j} \\
G_{i n}=\delta_{s t} B_{i}^{s} B_{n}^{t}+\delta_{a b} B_{i}^{a} B_{n}^{b}=0 \\
G_{m j}=\delta_{s t} B_{m}^{s} B_{j}^{t}+\delta_{a b} B_{m}^{a} B_{j}^{b}=0 \\
G_{m n}=B_{m}^{\mathfrak{D}-1} B_{n}^{\mathfrak{D}-1}+B_{m}^{\mathfrak{D}} B_{n}^{\mathfrak{D}}
\end{array},\left\{\begin{array}{l}
G^{i j}=\delta^{s t} C_{s}^{i} C_{t}^{j}=\delta^{s t} \delta_{s}^{i} \delta_{t}^{j}=\delta^{i j} \\
G^{i n}=\delta^{s t} C_{s}^{i} C_{t}^{n}=0 \\
G^{m j}=\delta^{s t} C_{s}^{m} C_{t}^{j}=0 \\
G^{m n}=C_{\mathfrak{D}-1}^{m} C_{\mathfrak{D}-1}^{n}+C_{\mathfrak{D}}^{m} C_{\mathfrak{D}}^{n}
\end{array} .\right.\right.
$$

Calculate the simple connection of $f$, that is $\Lambda_{N P}^{M} \triangleq \frac{1}{2} C_{A}^{M}\left(\frac{\partial B_{N}^{A}}{\partial x^{P}}+\frac{\partial B_{P}^{A}}{\partial x^{N}}\right)$ and $\Lambda_{M N P} \triangleq G_{M M^{\prime}} \Lambda_{N P}^{M^{\prime}}$, then we obtain

$$
\left\{\begin{array} { l } 
{ \Lambda _ { N P } ^ { i } = 0 } \\
{ \Lambda _ { j k } ^ { m } = 0 } \\
{ \Lambda _ { n P } ^ { m } = \frac { 1 } { 2 } C _ { a } ^ { m } ( \frac { \partial B _ { n } ^ { a } } { \partial x ^ { P } } + \frac { \partial B _ { P } ^ { a } } { \partial x ^ { n } } ) } \\
{ \Lambda _ { N p } ^ { m } = \frac { 1 } { 2 } C _ { a } ^ { m } ( \frac { \partial B _ { N } ^ { a } } { \partial x ^ { p } } + \frac { \partial B _ { p } ^ { a } } { \partial x ^ { N } } ) }
\end{array} \quad \left\{\begin{array}{l}
\Lambda_{i N P}=G_{i M^{\prime}} \Lambda_{N P}^{M^{\prime}}=G_{i i^{\prime}} \Lambda_{N P}^{i^{\prime}}=0 \\
\Lambda_{m j k}=G_{m M^{\prime}} \Lambda_{j k}^{M^{\prime}}=G_{m m^{\prime}} \Lambda_{j k}^{m^{\prime}}=0 \\
\Lambda_{m n P}=\frac{1}{2} \delta_{a b} B_{m}^{b}\left(\frac{\partial B_{n}^{a}}{\partial x^{P}}+\frac{\partial B_{P}^{a}}{\partial x^{n}}\right) \\
\Lambda_{m N p}=\frac{1}{2} \delta_{a b} B_{m}^{b}\left(\frac{\partial B_{N}^{a}}{\partial x^{p}}+\frac{\partial B_{p}^{a}}{\partial x^{N}}\right)
\end{array}\right.\right.
$$

Calculate the coefficients of curvature of $f$, that is

$$
\begin{aligned}
& K_{n P Q}^{m} \triangleq \frac{\partial \Lambda_{n Q}^{m}}{\partial x^{P}}-\frac{\partial \Lambda_{n P}^{m}}{\partial x^{Q}}+\Lambda_{H P}^{m} \Lambda_{n Q}^{H}-\Lambda_{n P}^{H} \Lambda_{H Q}^{m}, \\
& K_{m n P Q} \triangleq G_{m M^{\prime}} K_{n P Q}^{M^{\prime}}=G_{m m^{\prime}} K_{n P Q}^{m^{\prime}},
\end{aligned}
$$

then we obtain

$$
\begin{aligned}
K_{(\mathfrak{D}-1)(\mathfrak{D}-1) P Q} & =\frac{\partial \Lambda_{(\mathfrak{D}-1)(\mathfrak{D}-1) Q}}{\partial x^{P}}-\frac{\partial \Lambda_{(\mathfrak{D}-1)(\mathfrak{D}-1) P}}{\partial x^{Q}}+G^{\mathfrak{D D}}\left(\Lambda_{(\mathfrak{D}-1) \mathfrak{D} P} \Lambda_{\mathfrak{D}(\mathfrak{D}-1) Q}-\Lambda_{\mathfrak{D}(\mathfrak{D}-1) P} \Lambda_{(\mathfrak{D}-1) \mathfrak{D} Q}\right) \\
K_{\mathfrak{D}(\mathfrak{D}-1) P Q}= & \frac{\partial \Lambda_{\mathfrak{D}(\mathfrak{D}-1) Q}}{\partial x^{P}}-\frac{\partial \Lambda_{\mathfrak{D}(\mathfrak{D}-1) P}}{\partial x^{Q}}+G^{\mathfrak{D} \mathfrak{D}}\left(\Lambda_{\mathfrak{D} \mathfrak{D} P} \Lambda_{\mathfrak{D}(\mathfrak{D}-1) Q}-\Lambda_{\mathfrak{D}(\mathfrak{D}-1) P} \Lambda_{\mathfrak{D} \mathfrak{D} Q}\right) \\
& +G^{(\mathfrak{D}-1)(\mathfrak{D}-1)}\left(\Lambda_{\mathfrak{D}(\mathfrak{D}-1) P} \Lambda_{(\mathfrak{D}-1)(\mathfrak{D}-1) Q}-\Lambda_{(\mathfrak{D}-1)(\mathfrak{D}-1) P} \Lambda_{\mathfrak{D}(\mathfrak{D}-1) Q}\right) \\
K_{(\mathfrak{D}-1) \mathfrak{D} P Q}= & \frac{\partial \Lambda_{(\mathfrak{D}-1) \mathfrak{D} Q}}{\partial x^{P}}-\frac{\partial \Lambda_{(\mathfrak{D}-1) \mathfrak{D} P}}{\partial x^{Q}}+G^{\mathfrak{D D}}\left(\Lambda_{(\mathfrak{D}-1) \mathfrak{D} P} \Lambda_{\mathfrak{D} \mathfrak{D} Q}-\Lambda_{\mathfrak{D} \mathfrak{D} P} \Lambda_{(\mathfrak{D}-1) \mathfrak{D} Q}\right) \\
& +G^{(\mathfrak{D}-1)(\mathfrak{D}-1)}\left(\Lambda_{(\mathfrak{D}-1)(\mathfrak{D}-1) P} \Lambda_{(\mathfrak{D}-1) \mathfrak{D} Q}-\Lambda_{(\mathfrak{D}-1) \mathfrak{D} P} \Lambda_{(\mathfrak{D}-1)(\mathfrak{D}-1) Q}\right) \\
K_{\mathfrak{D} \mathfrak{D} P Q}= & \frac{\partial \Lambda_{\mathfrak{D} \mathfrak{D} Q}}{\partial x^{P}}-\frac{\partial \Lambda_{\mathfrak{D} \mathfrak{D} P}}{\partial x^{Q}}+G^{(\mathfrak{D}-1)(\mathfrak{D}-1)}\left(\Lambda_{\mathfrak{D}(\mathfrak{D}-1) P} \Lambda_{(\mathfrak{D}-1) \mathfrak{D} Q}-\Lambda_{(\mathfrak{D}-1) \mathfrak{D} P} \Lambda_{\mathfrak{D}(\mathfrak{D}-1) Q}\right)
\end{aligned}
$$

Hence,

$$
\begin{aligned}
B_{P Q} & \triangleq \frac{1}{\sqrt{2}}\left(K_{\mathfrak{D D P} Q}+K_{(\mathfrak{D}-1)(\mathfrak{D}-1) P Q}\right) \\
& =\frac{1}{\sqrt{2}} \frac{\partial\left(\Lambda_{\mathfrak{D} \mathfrak{D} Q}+\Lambda_{(\mathfrak{D}-1)(\mathfrak{D}-1) Q}\right)}{\partial x^{P}}-\frac{1}{\sqrt{2}} \frac{\partial\left(\Lambda_{\mathfrak{D} \mathfrak{D} P}+\Lambda_{(\mathfrak{D}-1)(\mathfrak{D}-1) P}\right)}{\partial x^{Q}}=\frac{\partial B_{Q}}{\partial x^{P}}-\frac{\partial B_{P}}{\partial x^{Q}} .
\end{aligned}
$$




$$
\begin{aligned}
F_{P Q}^{3} & \triangleq \frac{1}{\sqrt{2}}\left(K_{\mathfrak{D} \mathfrak{D} P Q}-K_{(\mathfrak{D}-1)(\mathfrak{D}-1) P Q}\right) \\
& =\frac{1}{\sqrt{2}}\left(\frac{\partial \Lambda_{\mathfrak{D} \mathfrak{D} Q}}{\partial x^{P}}-\frac{\partial \Lambda_{\mathfrak{D} \mathfrak{D} P}}{\partial x^{Q}}+G^{(\mathfrak{D}-1)(\mathfrak{D}-1)}\left(\Lambda_{\mathfrak{D}(\mathfrak{D}-1) P} \Lambda_{(\mathfrak{D}-1) \mathfrak{D} Q}-\Lambda_{(\mathfrak{D}-1) \mathfrak{D} P} \Lambda_{\mathfrak{D}(\mathfrak{D}-1) Q}\right)\right) \\
& -\frac{1}{\sqrt{2}}\left(\frac{\partial \Lambda_{(\mathfrak{D}-1)(\mathfrak{D}-1) Q}}{\partial x^{P}}-\frac{\partial \Lambda_{(\mathfrak{D}-1)(\mathfrak{D}-1) P}}{\partial x^{Q}}+G^{\mathfrak{D} \mathfrak{D}}\left(\Lambda_{(\mathfrak{D}-1) \mathfrak{D} P} \Lambda_{\mathfrak{D}(\mathfrak{D}-1) Q}-\Lambda_{\mathfrak{D}(\mathfrak{D}-1) P} \Lambda_{(\mathfrak{D}-1) \mathfrak{D} Q}\right)\right) \\
& =\frac{\partial A_{Q}^{3}}{\partial x^{P}}-\frac{\partial A_{P}^{3}}{\partial x^{Q}}+g\left(\Lambda_{\mathfrak{D}(\mathfrak{D}-1) P} \Lambda_{(\mathfrak{D}-1) \mathfrak{D} Q}-\Lambda_{(\mathfrak{D}-1) \mathfrak{D} P} \Lambda_{\mathfrak{D}(\mathfrak{D}-1) Q}\right) \\
& =\frac{\partial A_{Q}^{3}}{\partial x^{P}}-\frac{\partial A_{P}^{3}}{\partial x^{Q}}+g\left(A_{P}^{1} A_{Q}^{2}-A_{P}^{2} A_{Q}^{1}\right) .
\end{aligned}
$$

Similarly we also obtain $F_{P Q}^{1}=\frac{\partial A_{Q}^{1}}{\partial x^{P}}-\frac{\partial A_{P}^{1}}{\partial x^{Q}}+g\left(A_{P}^{2} A_{Q}^{3}-A_{P}^{3} A_{Q}^{2}\right)$ and $F_{P Q}^{2}=\frac{\partial A_{Q}^{2}}{\partial x^{P}}-\frac{\partial A_{P}^{2}}{\partial x^{Q}}-$ $g\left(A_{P}^{3} A_{Q}^{1}-A_{P}^{1} A_{Q}^{3}\right)$.

Remark 5.1. Comparing the above conclusion and $U(1) \times S U(2)$ principal bundle theory, we know this proposition indicates that the reference-system $f$ indeed can describe weak and electromagnetic field.

The following proposition shows an advantage of affine connection representation, that is to say, affine connection representation implies the chiral asymmetry of weak interaction, but $U(1) \times S U(2)$ principal bundle connection representation cannot imply it automatically.

Definition 5.2. Suppose $f$ and $g$ both satisfy Definition 5.1. According to Definition 3.2.1 and Definition 3.5.1, let $\rho_{m n}$ of $f$ evolve on $(M, g)$. Then $l \triangleq\left(\rho_{(\mathfrak{D}-1)(\mathfrak{D}-1)}, \rho_{\mathfrak{D D}}\right)$ is called an electric charged lepton, and $\nu \triangleq\left(\rho_{\mathfrak{D}(\mathfrak{D}-1)}, \rho_{(\mathfrak{D}-1) \mathfrak{D}}\right)$ is called a neutrino. $l$ and $\nu$ are collectively called leptons, denoted by $L$. And $L \frac{1}{\sqrt{2}}\left(\begin{array}{l}1 \\ 1\end{array}\right)$ is called a left-handed lepton, $L \frac{1}{\sqrt{2}}\left(\begin{array}{c}1 \\ -1\end{array}\right)$ is called a righthanded lepton, denoted by

$$
\left\{\begin{array} { l } 
{ l _ { L } \triangleq \frac { 1 } { \sqrt { 2 } } ( \rho _ { ( \mathfrak { D } - 1 ) ( \mathfrak { D } - 1 ) } + \rho _ { \mathfrak { D } \mathfrak { D } } ) , } \\
{ l _ { R } \triangleq \frac { 1 } { \sqrt { 2 } } ( \rho _ { ( \mathfrak { D } - 1 ) ( \mathfrak { D } - 1 ) } - \rho _ { \mathfrak { D } \mathfrak { D } } ) , }
\end{array} \quad \left\{\begin{array}{l}
\nu_{L} \triangleq \frac{1}{\sqrt{2}}\left(\rho_{\mathfrak{D}(\mathfrak{D}-1)}+\rho_{(\mathfrak{D}-1) \mathfrak{D}}\right) \\
\nu_{R} \triangleq \frac{1}{\sqrt{2}}\left(\rho_{\mathfrak{D}(\mathfrak{D}-1)}-\rho_{(\mathfrak{D}-1) \mathfrak{D}}\right) .
\end{array}\right.\right.
$$

On $(M, g)$ we define

$$
\left\{\begin{array} { l } 
{ W _ { P } ^ { 1 } \triangleq \frac { 1 } { \sqrt { 2 } } ( \Gamma _ { ( \mathfrak { D } - 1 ) \mathfrak { D } P } + \Gamma _ { \mathfrak { D } ( \mathfrak { D } - 1 ) P } ) , } \\
{ W _ { P } ^ { 2 } \triangleq \frac { 1 } { \sqrt { 2 } } ( \Gamma _ { ( \mathfrak { D } - 1 ) \mathfrak { D } P } - \Gamma _ { \mathfrak { D } ( \mathfrak { D } - 1 ) P } ) , }
\end{array} \quad \left\{\begin{array}{l}
Z_{P} \triangleq \frac{1}{\sqrt{2}}\left(\Gamma_{(\mathfrak{D}-1)(\mathfrak{D}-1) P}+\Gamma_{\mathfrak{D D} P}\right) \\
A_{P} \triangleq \frac{1}{\sqrt{2}}\left(\Gamma_{(\mathfrak{D}-1)(\mathfrak{D}-1) P}-\Gamma_{\mathfrak{D} \mathfrak{D} P}\right)
\end{array}\right.\right.
$$

and say the intrinsic geometric property $A_{P}$ is the electromagnetic potential, $Z_{P}$ is $\mathbf{Z}$ potential, $W_{P}^{1}$ and $W_{P}^{2}$ are $\mathbf{W}$ potentials. Then denote

$$
\left\{\begin{array} { l } 
{ W _ { P } ^ { + } \triangleq \frac { 1 } { \sqrt { 2 } } ( W _ { P } ^ { 1 } - i W _ { P } ^ { 2 } ) , } \\
{ W _ { P } ^ { - } \triangleq \frac { 1 } { \sqrt { 2 } } ( W _ { P } ^ { 1 } + i W _ { P } ^ { 2 } ) , }
\end{array} \quad \left\{\begin{array} { l } 
{ l _ { L } ^ { + } = \frac { 1 } { \sqrt { 2 } } ( l _ { L } - i l _ { R } ) , } \\
{ l _ { L } ^ { - } = \frac { 1 } { \sqrt { 2 } } ( l _ { L } + i l _ { R } ) , }
\end{array} \quad \left\{\begin{array}{l}
l_{R}^{+} \triangleq \frac{1}{\sqrt{2}}\left(l_{R}-i l_{L}\right), \\
l_{R}^{-} \triangleq \frac{1}{\sqrt{2}}\left(l_{R}+i l_{L}\right) .
\end{array}\right.\right.\right.
$$


Proposition 5.2. If $(M, g)$ satisfies the symmetry condition $\Gamma_{(\mathfrak{D}-1) \mathfrak{D} P}=\Gamma_{\mathfrak{D}(\mathfrak{D}-1) P}$, then the intrinsic geometric properties $l$ and $\nu$ of $f$ satisfy the following conclusions on $(M, g)$.

$$
\left\{\begin{array}{l}
l_{L ; P}=\partial_{P} l_{L}-g l_{L} Z_{P}-g l_{R} A_{P}-g \nu_{L} W_{P}^{1} \\
l_{R ; P}=\partial_{P} l_{R}-g l_{R} Z_{P}-g l_{L} A_{P} \\
\nu_{L ; P}=\partial_{P} \nu_{L}-g \nu_{L} Z_{P}-g l_{L} W_{P}^{1} \\
\nu_{R ; P}=\partial_{P} \nu_{R}-g \nu_{R} Z_{P} .
\end{array}\right.
$$

Proof. Due to $\rho_{m n ; P}=\partial_{P} \rho_{m n}-\rho_{H n} \Gamma_{m P}^{H}-\rho_{m H} \Gamma_{n P}^{H}=\partial_{P} \rho_{m n}-\rho_{h n} \Gamma_{m P}^{h}-\rho_{m h} \Gamma_{n P}^{h}$, we have

$$
\begin{gathered}
\left\{\begin{array}{l}
\rho_{(\mathfrak{D}-1)(\mathfrak{D}-1) ; P}=\partial_{P} \rho_{(\mathfrak{D}-1)(\mathfrak{D}-1)}-2 \rho_{(\mathfrak{D}-1)(\mathfrak{D}-1)} \Gamma_{(\mathfrak{D}-1) P}^{\mathfrak{D}-1}-\left(\rho_{\mathfrak{D}(\mathfrak{D}-1)}+\rho_{(\mathfrak{D}-1) \mathfrak{D}}\right) \Gamma_{(\mathfrak{D}-1) P}^{\mathfrak{D}}, \\
\rho_{\mathfrak{D} \mathfrak{D} ; P}=\partial_{P} \rho_{\mathfrak{D} \mathfrak{D}}-2 \rho_{\mathfrak{D} \mathfrak{D}} \Gamma_{\mathfrak{D} P}^{\mathfrak{D}}-\left(\rho_{\mathfrak{D}(\mathfrak{D}-1)}+\rho_{(\mathfrak{D}-1) \mathfrak{D}}\right) \Gamma_{\mathfrak{D} P}^{\mathfrak{D}-1} \\
\rho_{\mathfrak{D}(\mathfrak{D}-1) ; P}=\partial_{P} \rho_{\mathfrak{D}(\mathfrak{D}-1)}-\left(\rho_{(\mathfrak{D}-1)(\mathfrak{D}-1)} \Gamma_{\mathfrak{D} P}^{\mathfrak{D}-1}+\rho_{\mathfrak{D} \mathfrak{D}} \Gamma_{(\mathfrak{D}-1) P}^{\mathfrak{D}}\right)-\rho_{\mathfrak{D}(\mathfrak{D}-1)}\left(\Gamma_{(\mathfrak{D}-1) P}^{\mathfrak{D}-1}+\Gamma_{\mathfrak{D} P}^{\mathfrak{D}}\right), \\
\rho_{(\mathfrak{D}-1) \mathfrak{D} ; P}=\partial_{P} \rho_{(\mathfrak{D}-1) \mathfrak{D}}-\left(\rho_{(\mathfrak{D}-1)(\mathfrak{D}-1)} \Gamma_{\mathfrak{D} P}^{\mathfrak{D}-1}+\rho_{\mathfrak{D} \mathfrak{D}} \Gamma_{(\mathfrak{D}-1) P}^{\mathfrak{D}}\right)-\rho_{(\mathfrak{D}-1) \mathfrak{D}}\left(\Gamma_{(\mathfrak{D}-1) P}^{\mathfrak{D}-1}+\Gamma_{\mathfrak{D} P}^{\mathfrak{D}}\right) .
\end{array}\right. \\
\Rightarrow\left\{\begin{array}{l}
l_{L ; P}=\partial_{P} l_{L}-\sqrt{2} \rho_{(\mathfrak{D}-1)(\mathfrak{D}-1)} \Gamma_{(\mathfrak{D}-1) P}^{\mathfrak{D}-1}-\sqrt{2} \rho_{\mathfrak{D} \mathfrak{D}} \Gamma_{\mathfrak{D} P}^{\mathfrak{D}}-g \nu_{L} W_{P}^{1} \\
l_{R ; P}=\partial_{P} l_{R}-\sqrt{2} \rho_{(\mathfrak{D}-1)(\mathfrak{D}-1)} \Gamma_{(\mathfrak{D}-1) P}^{\mathcal{D}-1}+\sqrt{2} \rho_{\mathfrak{D} \mathfrak{D}} \Gamma_{\mathfrak{D} P}^{\mathfrak{D}}, \\
\nu_{L ; P}=\partial_{P} \nu_{L}-g l_{L} W_{P}^{1}-\nu_{L}\left(\Gamma_{(\mathfrak{D}-1) P}^{\mathfrak{D}-1}+\Gamma_{\mathfrak{D} P}^{\mathfrak{D}}\right) \\
\nu_{R ; P}=\partial_{P} \nu_{R}-\nu_{R}\left(\Gamma_{(\mathfrak{D}-1) P}^{\mathfrak{D}-1}+\Gamma_{\mathfrak{D} P}^{\mathfrak{D}}\right)
\end{array}\right. \\
\Rightarrow\left\{\begin{array}{l}
l_{L ; P}=\partial_{P} l_{L}-g l_{L} Z_{P}-g l_{R} A_{P}-g \nu_{L} W_{P}^{1}, \\
l_{R ; P}=\partial_{P} l_{R}-g l_{R} Z_{P}-g l_{L} A_{P}, \\
\nu_{L ; P}=\partial_{P} \nu_{L}-g \nu_{L} Z_{P}-g l_{L} W_{P}^{1}, \\
\nu_{R ; P}=\partial_{P} \nu_{R}-g \nu_{R} Z_{P} .
\end{array}\right.
\end{gathered}
$$

Remark 5.2. The above proposition shows that the chiral asymmetry of leptons is a direct mathematical result of affine connection representation, and that it is not necessary to be postulated artificially like that in $U_{Y}(1) \times S U_{L}(2)$ theory.

Discussion 5.1. According to section 2.4, the intrinsic geometry is the largest geometry on geometric manifold, and its geometric properties are the richest, so that any irregular smooth shape can be precisely characterized by $B_{M}^{A}$ and $C_{A}^{M}$. Its equivalent transformation group is the trivial subgroup $\{e\}$ that has only one element. We notice that:

(1) Principal bundle connection representation starts from a very large group, and reduces symmetries in way of "symmetry breaking" to approach the target geometry.

(2) Affine connection representation starts from the smallest group $\{e\}$, and adds symmetries in way of "symmetry condition" to approach the target geometry.

Such two ways must lead to the same destination. They both go towards the same specific geometry. 
Remark 5.3. Proposition 5.2 shows that:

(1) In affine connection representation of gauge fields, the coupling constant $g$ is possessed of a geometric meaning, that is in fact the metric of internal space. But it does not have such a clear geometric meaning in $U(1) \times S U(2)$ principal bundle connection representation.

(2) At the most fundamental level, the coupling constant of $Z_{P}$ and that of $A_{P}$ are equal, i.e.

$$
g_{Z}=g_{A}=g .
$$

Suppose there is a kind of medium. $Z$ boson and photon move in it. Suppose $Z$ field has interaction with the medium, but electromagnetic field $A$ has no interaction with the medium. Then we have coupling constants

$$
\tilde{g}_{Z} \neq \tilde{g}_{A}
$$

in the medium, and the Weinberg angle appears.

It is quite reasonable to consider a Higgs boson as a zero-spin pair of neutrinos, because in Standard Model, Higgs boson only couples with $Z$ field and $W$ field, but does not couple with electromagnetic field and gluon field. If so, Higgs boson would lose its fundamentality and it would not have enough importance in a theory at the most fundamental level.

(3) The mixing of leptons of three generations do not appear in Proposition 5.2, but it can spontaneously appear in Proposition 7.1 due to the affine connection representation of the gauge field that is given by Definition 7.1.

\section{Affine connection representation of the gauge field of strong interaction}

Definition 6.1. Suppose a geometric manifold $(M, f)$ satisfies $\mathfrak{D}=r+3=6 . \forall p \in M$, the coordinate representation of $f(p)$ takes the form of

$$
\xi^{a}=\xi^{a}\left(x^{m}\right), \quad \xi^{s}=\delta_{i}^{s} x^{i}, \quad(s, i=1,2,3 ; a, m=4,5,6),
$$

and $f$ satisfies the internal standard conditions (55) of Definition 4.6.1(1) and

$$
G^{(\mathfrak{D}-2)(\mathfrak{D}-2)}=G^{(\mathfrak{D}-1)(\mathfrak{D}-1)}=G^{\mathfrak{D} \mathfrak{D}} .
$$

We say $f$ is a strong interaction field.

Definition 6.2. Suppose $f$ and $g$ both satisfy Definition 6.1. According to Definition 3.2.1 and Definition 3.5.1, let $\rho_{m n}$ of $f$ evolve on $(M, g)$. Define

$$
\left\{\begin{array} { l } 
{ d _ { 1 } \triangleq ( \rho _ { ( \mathfrak { D } - 2 ) ( \mathfrak { D } - 2 ) } , \rho _ { ( \mathfrak { D } - 1 ) ( \mathfrak { D } - 1 ) } ) , } \\
{ d _ { 2 } \triangleq ( \rho _ { ( \mathfrak { D } - 1 ) ( \mathfrak { D } - 1 ) } , \rho _ { \mathfrak { D } \mathfrak { D } } ) , } \\
{ d _ { 3 } \triangleq ( \rho _ { \mathfrak { D } \mathfrak { D } } , \rho _ { ( \mathfrak { D } - 2 ) ( \mathfrak { D } - 2 ) } ) , }
\end{array} \quad \left\{\begin{array}{l}
u_{1} \triangleq\left(\rho_{(\mathfrak{D}-2)(\mathfrak{D}-1)}, \rho_{(\mathfrak{D}-1)(\mathfrak{D}-2)}\right), \\
u_{2} \triangleq\left(\rho_{(\mathfrak{D}-1) \mathfrak{D}}, \rho_{\mathfrak{D}(\mathfrak{D}-1)}\right), \\
u_{3} \triangleq\left(\rho_{\mathfrak{D}(\mathfrak{D}-2)}, \rho_{(\mathfrak{D}-2) \mathfrak{D}}\right) .
\end{array}\right.\right.
$$

We say $d_{1}$ and $u_{1}$ are red color charges, $d_{2}$ and $u_{2}$ are blue color charges, $d_{3}$ and $u_{3}$ are green color charges. Then $d_{1}, d_{2}, d_{3}$ are called down-type color charges, uniformly denoted by $d$, and $u_{1}, u_{2}, u_{3}$ are called up-type color charges, uniformly denoted by $u$. $d$ and $u$ are collectively called 
color charges, denoted by $q$. We say $q \frac{1}{\sqrt{2}}\left(\begin{array}{l}1 \\ 1\end{array}\right)$ is a left-handed color charge, and $q \frac{1}{\sqrt{2}}\left(\begin{array}{c}1 \\ -1\end{array}\right)$ is a right-handed color charge. They are

$$
\begin{aligned}
& \left\{\begin{array}{l}
d_{1 L} \triangleq \frac{1}{\sqrt{2}}\left(\rho_{(\mathfrak{D}-2)(\mathfrak{D}-2)}+\rho_{(\mathfrak{D}-1)(\mathfrak{D}-1)}\right), \\
d_{2 L} \triangleq \frac{1}{\sqrt{2}}\left(\rho_{(\mathfrak{D}-1)(\mathfrak{D}-1)}+\rho_{\mathfrak{D} \mathfrak{D}}\right), \\
d_{3 L} \triangleq \frac{1}{\sqrt{2}}\left(\rho_{\mathfrak{D} \mathfrak{D}}+\rho_{(\mathfrak{D}-2)(\mathfrak{D}-2)}\right),
\end{array}\right. \\
& \left\{\begin{array}{l}
u_{1 L} \triangleq \frac{1}{\sqrt{2}}\left(\rho_{(\mathfrak{D}-2)(\mathfrak{D}-1)}+\rho_{(\mathfrak{D}-1)(\mathfrak{D}-2)}\right), \\
d_{2 R} \triangleq \frac{1}{\sqrt{2}}\left(\rho_{(\mathfrak{D}-2)(\mathfrak{D}-2)}-\rho_{(\mathfrak{D}-1)(\mathfrak{D}-1)}\right) \\
d_{3 R} \triangleq \frac{1}{\sqrt{2}}\left(\rho_{(\mathfrak{D}-1)(\mathfrak{D}-1)}-\rho_{\mathfrak{D} \mathfrak{D}}\right) \\
u_{2 L} \triangleq \frac{1}{\sqrt{2}}\left(\rho_{(\mathfrak{D}-1) \mathfrak{D}}+\rho_{(\mathfrak{D}-2)(\mathfrak{D}-2)}\right), \\
u_{3 L} \triangleq \frac{1}{\sqrt{2}}\left(\rho_{\mathfrak{D}(\mathfrak{D}-1)}\right),
\end{array}\right.
\end{aligned}
$$

On $(M, g)$ we denote

$$
\begin{aligned}
& g_{s} \triangleq \sqrt{\left(G^{(\mathfrak{D}-1)(\mathfrak{D}-1)}\right)^{2}+\left(G^{\mathfrak{D} \mathfrak{D}}\right)^{2}}=\sqrt{\left(G^{(\mathfrak{D}-1)(\mathfrak{D}-1)}\right)^{2}+\left(G^{(\mathfrak{D}-2)(\mathfrak{D}-2)}\right)^{2}} \\
& =\sqrt{\left(G^{(\mathfrak{D}-2)(\mathfrak{D}-2)}\right)^{2}+\left(G^{\mathfrak{D} \mathfrak{D}}\right)^{2}} \text {. } \\
& \left\{\begin{array} { l } 
{ U _ { P } ^ { 1 } \triangleq \frac { 1 } { \sqrt { 2 } } ( \Gamma _ { ( \mathfrak { D } - 2 ) ( \mathfrak { D } - 2 ) P } + \Gamma _ { ( \mathfrak { D } - 1 ) ( \mathfrak { D } - 1 ) P } ) , } \\
{ V _ { P } ^ { 1 } \triangleq \frac { 1 } { \sqrt { 2 } } ( \Gamma _ { ( \mathfrak { D } - 2 ) ( \mathfrak { D } - 2 ) P } - \Gamma _ { ( \mathfrak { D } - 1 ) ( \mathfrak { D } - 1 ) P } ) , }
\end{array} \quad \left\{\begin{array}{l}
X_{P}^{23} \triangleq \frac{1}{\sqrt{2}}\left(\Gamma_{(\mathfrak{D}-2)(\mathfrak{D}-1) P}+\Gamma_{(\mathfrak{D}-1)(\mathfrak{D}-2) P}\right), \\
Y_{P}^{23} \triangleq \frac{1}{\sqrt{2}}\left(\Gamma_{(\mathfrak{D}-2)(\mathfrak{D}-1) P}-\Gamma_{(\mathfrak{D}-1)(\mathfrak{D}-2) P}\right),
\end{array}\right.\right. \\
& \left\{\begin{array} { l } 
{ U _ { P } ^ { 2 } \triangleq \frac { 1 } { \sqrt { 2 } } ( \Gamma _ { ( \mathfrak { D } - 1 ) ( \mathfrak { D } - 1 ) P } + \Gamma _ { \mathfrak { D D } P } ) , } \\
{ V _ { P } ^ { 2 } \triangleq \frac { 1 } { \sqrt { 2 } } ( \Gamma _ { ( \mathfrak { D } - 1 ) ( \mathfrak { D } - 1 ) P } - \Gamma _ { \mathfrak { D } \mathfrak { D } P } ) , }
\end{array} \quad \left\{\begin{array}{l}
X_{P}^{31} \triangleq \frac{1}{\sqrt{2}}\left(\Gamma_{(\mathfrak{D}-1) \mathfrak{D} P}+\Gamma_{\mathfrak{D}(\mathfrak{D}-1) P}\right) \\
Y_{P}^{31} \triangleq \frac{1}{\sqrt{2}}\left(\Gamma_{(\mathfrak{D}-1) \mathfrak{D} P}-\Gamma_{\mathfrak{D}(\mathfrak{D}-1) P}\right)
\end{array}\right.\right. \\
& \left\{\begin{array} { l } 
{ U _ { P } ^ { 3 } \triangleq \frac { 1 } { \sqrt { 2 } } ( \Gamma _ { \mathfrak { D } \mathfrak { D } P } + \Gamma _ { ( \mathfrak { D } - 2 ) ( \mathfrak { D } - 2 ) P } ) , } \\
{ V _ { P } ^ { 3 } \triangleq \frac { 1 } { \sqrt { 2 } } ( \Gamma _ { \mathfrak { D } \mathfrak { D } P } - \Gamma _ { ( \mathfrak { D } - 2 ) ( \mathfrak { D } - 2 ) P } ) , }
\end{array} \quad \left\{\begin{array}{l}
X_{P}^{12} \triangleq \frac{1}{\sqrt{2}}\left(\Gamma_{\mathfrak{D}(\mathfrak{D}-2) P}+\Gamma_{(\mathfrak{D}-2) \mathfrak{D} P}\right) \\
Y_{P}^{12} \triangleq \frac{1}{\sqrt{2}}\left(\Gamma_{\mathfrak{D}(\mathfrak{D}-2) P}-\Gamma_{(\mathfrak{D}-2) \mathfrak{D} P}\right)
\end{array}\right.\right.
\end{aligned}
$$

We notice that there are just only three independent ones in $U_{P}^{1}, U_{P}^{2}, U_{P}^{3}, V_{P}^{1}, V_{P}^{2}$ and $V_{P}^{3}$. Without loss of generality, let

$$
\left\{\begin{array} { l } 
{ R _ { P } \triangleq a _ { R } U _ { P } ^ { 1 } + b _ { R } U _ { P } ^ { 2 } + c _ { R } U _ { P } ^ { 3 } , } \\
{ S _ { P } \triangleq a _ { S } U _ { P } ^ { 1 } + b _ { S } U _ { P } ^ { 2 } + c _ { S } U _ { P } ^ { 3 } , } \\
{ T _ { P } \triangleq a _ { T } U _ { P } ^ { 1 } + b _ { T } U _ { P } ^ { 2 } + c _ { T } U _ { P } ^ { 3 } , }
\end{array} \quad \left\{\begin{array}{l}
U_{P}^{1} \triangleq \alpha_{R} R_{P}+\alpha_{S} S_{P}+\alpha_{T} T_{P} \\
U_{P}^{2} \triangleq \beta_{R} R_{P}+\beta_{S} S_{P}+\beta_{T} T_{P} \\
U_{P}^{3} \triangleq \gamma_{R} R_{P}+\gamma_{S} S_{P}+\gamma_{T} T_{P}
\end{array}\right.\right.
$$

where the coefficients matrix is non-singular. 
Proposition 6.1. Let $\lambda_{a}(a=1,2, \cdots, 8)$ be the Gell-Mann matrices, and $T_{a} \triangleq \frac{1}{2} \lambda_{a}$ the generators of $S U(3)$ group. When $(M, g)$ satisfies the symmetry condition $\Gamma_{(\mathfrak{D}-2)(\mathfrak{D}-2) P}+\Gamma_{(\mathfrak{D}-1)(\mathfrak{D}-1) P}+$ $\Gamma_{\mathfrak{D D P}}=0$, denote

$$
A_{P} \triangleq \frac{1}{2}\left(\begin{array}{ccc}
A_{P}^{11} & A_{P}^{12} & A_{P}^{13} \\
A_{P}^{21} & A_{P}^{22} & A_{P}^{23} \\
A_{P}^{31} & A_{P}^{32} & A_{P}^{33}
\end{array}\right), \begin{aligned}
& A_{P}^{11} \triangleq S_{P}+\frac{1}{\sqrt{6}} T_{P}, \quad A_{P}^{12} \triangleq X_{P}^{12}-i Y_{P}^{12}, \quad A_{P}^{12}+i Y_{P}^{12}, \quad A_{P}^{22} \triangleq-S_{P}+\frac{1}{\sqrt{6}} T_{P}, \quad A_{P}^{23} \triangleq X_{P}^{23}-i Y_{P}^{23}, \\
& A_{P}^{31} \triangleq X_{P}^{31}+i Y_{P}^{31}, \quad A_{P}^{32} \triangleq X_{P}^{23}+i Y_{P}^{23}, \quad A_{P}^{33} \triangleq-\frac{2}{\sqrt{6}} T_{P} .
\end{aligned}
$$

Thus, $A_{P}=T_{a} A_{P}^{a}$ if and only if $A_{P}^{1}=X_{P}^{12}, A_{P}^{2}=Y_{P}^{12}, A_{P}^{3}=S_{P}, A_{P}^{4}=X_{P}^{31}, A_{P}^{5}=Y_{P}^{31}$, $A_{P}^{6}=X_{P}^{23}, A_{P}^{7}=Y_{P}^{23}$ and $A_{P}^{8}=T_{P}$.

Proof. We just need to substitute the Gell-Mann matrices

$$
\begin{gathered}
\lambda_{1} \triangleq\left(\begin{array}{lll}
0 & 1 & 0 \\
1 & 0 & 0 \\
0 & 0 & 0
\end{array}\right), \lambda_{2} \triangleq\left(\begin{array}{ccc}
0 & -i & 0 \\
i & 0 & 0 \\
0 & 0 & 0
\end{array}\right), \lambda_{3} \triangleq\left(\begin{array}{ccc}
1 & 0 & 0 \\
0 & -1 & 0 \\
0 & 0 & 0
\end{array}\right), \lambda_{4} \triangleq\left(\begin{array}{ccc}
0 & 0 & 1 \\
0 & 0 & 0 \\
1 & 0 & 0
\end{array}\right), \\
\lambda_{5} \triangleq\left(\begin{array}{ccc}
0 & 0 & -i \\
0 & 0 & 0 \\
i & 0 & 0
\end{array}\right), \lambda_{6} \triangleq\left(\begin{array}{lll}
0 & 0 & 0 \\
0 & 0 & 1 \\
0 & 1 & 0
\end{array}\right), \lambda_{7} \triangleq\left(\begin{array}{ccc}
0 & 0 & 0 \\
0 & 0 & -i \\
0 & i & 0
\end{array}\right), \lambda_{8} \triangleq \frac{1}{\sqrt{6}}\left(\begin{array}{ccc}
1 & 0 & 0 \\
0 & 1 & 0 \\
0 & 0 & -2
\end{array}\right) .
\end{gathered}
$$

into $A_{P}=T_{a} A_{P}^{a}$ and directly verify them.

Remark 6.1. On one hand, the above proposition shows that Definition 6.1 is an affine connection representation of strong interaction field. It does not define the gauge potentials abstractly like QCD based on $S U(3)$ theory, but gives concrete intrinsic geometric constructions to the gauge potentials. On the other hand, the above proposition implies that if we take appropriate symmetry conditions, the algebraic properties of $S U(3)$ group can be described by the transformation group $G L(3, \mathbb{R})$ of internal space of $g$. In other words, the exponential map exp $: G L(3, \mathbb{R}) \rightarrow U(3),\left[B_{m}^{a}\right] \mapsto e^{i T_{a}^{m} B_{m}^{a}}$ defines a covering homomorphism, and $S U(3)$ is a subgroup of $U(3)$.

\section{Affine connection representation of the unified gauge field}

Definition 7.1. Suppose a geometric manifold $(M, f)$ satisfies $\mathfrak{D}=r+5=8$. $\forall p \in M$, the coordinate representation of $f(p)$ takes the form of

$$
\xi^{a}=\xi^{a}\left(x^{m}\right), \quad \xi^{s}=\delta_{i}^{s} x^{i}, \quad(s, i=1,2,3 ; a, m=4,5,6,7,8),
$$

and $f$ satisfies the internal standard conditions (55) of Definition 4.6.1(1) and

$$
G^{(\mathfrak{D}-4)(\mathfrak{D}-4)}=G^{(\mathfrak{D}-3)(\mathfrak{D}-3)}, \quad G^{(\mathfrak{D}-2)(\mathfrak{D}-2)}=G^{(\mathfrak{D}-1)(\mathfrak{D}-1)}=G^{\mathfrak{D D}} .
$$

We say $f$ is a typical unified gauge field. 
Definition 7.2. Suppose $f$ and $g$ both satisfy Definition 7.1. According to Definition 3.2.1 and Definition 3.5.1, let $\rho_{m n}$ of $f$ evolve on $(M, g)$. Define

$$
\left\{\begin{array} { l } 
{ l \triangleq ( \rho _ { ( \mathfrak { D } - 4 ) ( \mathfrak { D } - 4 ) } , \rho _ { ( \mathfrak { D } - 3 ) ( \mathfrak { D } - 3 ) } ) , } \\
{ d _ { 1 } \triangleq ( \rho _ { ( \mathfrak { D } - 2 ) ( \mathfrak { D } - 2 ) } , \rho _ { ( \mathfrak { D } - 1 ) ( \mathfrak { D } - 1 ) } ) , } \\
{ d _ { 2 } \triangleq ( \rho _ { ( \mathfrak { D } - 1 ) ( \mathfrak { D } - 1 ) } , \rho _ { \mathfrak { D } \mathfrak { D } } ) , } \\
{ d _ { 3 } \triangleq ( \rho _ { \mathfrak { D } \mathfrak { D } } , \rho _ { ( \mathfrak { D } - 2 ) ( \mathfrak { D } - 2 ) } ) , }
\end{array} \quad \left\{\begin{array}{l}
\nu \triangleq\left(\rho_{(\mathfrak{D}-3)(\mathfrak{D}-4)}, \rho_{(\mathfrak{D}-4)(\mathfrak{D}-3)}\right) \\
u_{1} \triangleq\left(\rho_{(\mathfrak{D}-2)(\mathfrak{D}-1)}, \rho_{(\mathfrak{D}-1)(\mathfrak{D}-2)}\right), \\
u_{2} \triangleq\left(\rho_{(\mathfrak{D}-1) \mathfrak{D}}, \rho_{\mathfrak{D}(\mathfrak{D}-1)}\right), \\
u_{3} \triangleq\left(\rho_{\mathfrak{D}(\mathfrak{D}-2)}, \rho_{(\mathfrak{D}-2) \mathfrak{D}}\right) .
\end{array}\right.\right.
$$

\section{And Denote}

$$
\begin{aligned}
& \left\{\begin{array} { l } 
{ l _ { L } \triangleq \frac { 1 } { \sqrt { 2 } } ( \rho _ { ( \mathfrak { D } - 4 ) ( \mathfrak { D } - 4 ) } + \rho _ { ( \mathfrak { D } - 3 ) ( \mathfrak { D } - 3 ) } ) , } \\
{ l _ { R } \triangleq \frac { 1 } { \sqrt { 2 } } ( \rho _ { ( \mathfrak { D } - 4 ) ( \mathfrak { D } - 4 ) } - \rho _ { ( \mathfrak { D } - 3 ) ( \mathfrak { D } - 3 ) } ) , }
\end{array} \quad \left\{\begin{array}{l}
\nu_{L} \triangleq \frac{1}{\sqrt{2}}\left(\rho_{(\mathfrak{D}-3)(\mathfrak{D}-4)}+\rho_{(\mathfrak{D}-4)(\mathfrak{D}-3)}\right), \\
\nu_{R} \triangleq \frac{1}{\sqrt{2}}\left(\rho_{(\mathfrak{D}-3)(\mathfrak{D}-4)}-\rho_{(\mathfrak{D}-4)(\mathfrak{D}-3)}\right),
\end{array}\right.\right. \\
& \left\{\begin{array} { l } 
{ d _ { 1 L } \triangleq \frac { 1 } { \sqrt { 2 } } ( \rho _ { ( \mathfrak { D } - 2 ) ( \mathfrak { D } - 2 ) } + \rho _ { ( \mathfrak { D } - 1 ) ( \mathfrak { D } - 1 ) } ) , } \\
{ d _ { 2 L } \triangleq \frac { 1 } { \sqrt { 2 } } ( \rho _ { ( \mathfrak { D } - 1 ) ( \mathfrak { D } - 1 ) } + \rho _ { \mathfrak { D } \mathfrak { D } } ) , } \\
{ d _ { 3 L } \triangleq \frac { 1 } { \sqrt { 2 } } ( \rho _ { \mathfrak { D } \mathfrak { D } } + \rho _ { ( \mathfrak { D } - 2 ) ( \mathfrak { D } - 2 ) } ) , }
\end{array} \quad \left\{\begin{array}{l}
d_{1 R} \triangleq \frac{1}{\sqrt{2}}\left(\rho_{(\mathfrak{D}-2)(\mathfrak{D}-2)}-\rho_{(\mathfrak{D}-1)(\mathfrak{D}-1)}\right), \\
d_{2 R} \triangleq \frac{1}{\sqrt{2}}\left(\rho_{(\mathfrak{D}-1)(\mathfrak{D}-1)}-\rho_{\mathfrak{D} \mathfrak{D}}\right), \\
d_{3 R} \triangleq \frac{1}{\sqrt{2}}\left(\rho_{\mathfrak{D} \mathfrak{D}}-\rho_{(\mathfrak{D}-2)(\mathfrak{D}-2)}\right),
\end{array}\right.\right. \\
& \left\{\begin{array} { l } 
{ u _ { 1 L } \triangleq \frac { 1 } { \sqrt { 2 } } ( \rho _ { ( \mathfrak { D } - 2 ) ( \mathfrak { D } - 1 ) } + \rho _ { ( \mathfrak { D } - 1 ) ( \mathfrak { D } - 2 ) } ) , } \\
{ u _ { 2 L } \triangleq \frac { 1 } { \sqrt { 2 } } ( \rho _ { ( \mathfrak { D } - 1 ) \mathfrak { D } } + \rho _ { \mathfrak { D } ( \mathfrak { D } - 1 ) } ) , } \\
{ u _ { 3 L } \triangleq \frac { 1 } { \sqrt { 2 } } ( \rho _ { \mathfrak { D } ( \mathfrak { D } - 2 ) } + \rho _ { ( \mathfrak { D } - 2 ) \mathfrak { D } } ) , }
\end{array} \quad \left\{\begin{array}{l}
u_{1 R} \triangleq \frac{1}{\sqrt{2}}\left(\rho_{(\mathfrak{D}-2)(\mathfrak{D}-1)}-\rho_{(\mathfrak{D}-1)(\mathfrak{D}-2)}\right), \\
u_{2 R} \triangleq \frac{1}{\sqrt{2}}\left(\rho_{(\mathfrak{D}-1) \mathfrak{D}}-\rho_{\mathfrak{D}(\mathfrak{D}-1)}\right), \\
u_{3 R} \triangleq \frac{1}{\sqrt{2}}\left(\rho_{\mathfrak{D}(\mathfrak{D}-2)}-\rho_{(\mathfrak{D}-2) \mathfrak{D}}\right) .
\end{array}\right.\right.
\end{aligned}
$$

On $(M, g)$ we denote

$$
\begin{aligned}
& \left\{\begin{aligned}
g \triangleq \sqrt{\left(G^{(\mathfrak{D}-4)(\mathfrak{D}-4)}\right)^{2}+\left(G^{(\mathfrak{D}-3)(\mathfrak{D}-3)}\right)^{2}} & \\
g_{s} \triangleq \sqrt{\left(G^{(\mathfrak{D}-1)(\mathfrak{D}-1)}\right)^{2}+\left(G^{\mathfrak{D} \mathfrak{D}}\right)^{2}} & =\sqrt{\left(G^{(\mathfrak{D}-1)(\mathfrak{D}-1)}\right)^{2}+\left(G^{(\mathfrak{D}-2)(\mathfrak{D}-2)}\right)^{2}} \\
& =\sqrt{\left(G^{(\mathfrak{D}-2)(\mathfrak{D}-2)}\right)^{2}+\left(G^{\mathfrak{D} \mathfrak{D}}\right)^{2}},
\end{aligned}\right. \\
& \left\{\begin{array} { l } 
{ Z _ { P } \triangleq \frac { 1 } { \sqrt { 2 } } ( \Gamma _ { ( \mathfrak { D } - 4 ) ( \mathfrak { D } - 4 ) P } + \Gamma _ { ( \mathfrak { D } - 3 ) ( \mathfrak { D } - 3 ) P } ) , } \\
{ A _ { P } \triangleq \frac { 1 } { \sqrt { 2 } } ( \Gamma _ { ( \mathfrak { D } - 4 ) ( \mathfrak { D } - 4 ) P } - \Gamma _ { ( \mathfrak { D } - 3 ) ( \mathfrak { D } - 3 ) P } ) , }
\end{array} \quad \left\{\begin{array}{l}
W_{P}^{1} \triangleq \frac{1}{\sqrt{2}}\left(\Gamma_{(\mathfrak{D}-4)(\mathfrak{D}-3) P}+\Gamma_{(\mathfrak{D}-3)(\mathfrak{D}-4) P}\right), \\
W_{P}^{2} \triangleq \frac{1}{\sqrt{2}}\left(\Gamma_{(\mathfrak{D}-4)(\mathfrak{D}-3) P}-\Gamma_{(\mathfrak{D}-3)(\mathfrak{D}-4) P}\right),
\end{array}\right.\right. \\
& \left\{\begin{array} { l } 
{ U _ { P } ^ { 1 } \triangleq \frac { 1 } { \sqrt { 2 } } ( \Gamma _ { ( \mathfrak { D } - 2 ) ( \mathfrak { D } - 2 ) P } + \Gamma _ { ( \mathfrak { D } - 1 ) ( \mathfrak { D } - 1 ) P } ) , } \\
{ V _ { P } ^ { 1 } \triangleq \frac { 1 } { \sqrt { 2 } } ( \Gamma _ { ( \mathfrak { D } - 2 ) ( \mathfrak { D } - 2 ) P } - \Gamma _ { ( \mathfrak { D } - 1 ) ( \mathfrak { D } - 1 ) P } ) , }
\end{array} \quad \left\{\begin{array}{l}
X_{P}^{23} \triangleq \frac{1}{\sqrt{2}}\left(\Gamma_{(\mathfrak{D}-2)(\mathfrak{D}-1) P}+\Gamma_{(\mathfrak{D}-1)(\mathfrak{D}-2) P}\right), \\
Y_{P}^{23} \triangleq \frac{1}{\sqrt{2}}\left(\Gamma_{(\mathfrak{D}-2)(\mathfrak{D}-1) P}-\Gamma_{(\mathfrak{D}-1)(\mathfrak{D}-2) P}\right),
\end{array}\right.\right.
\end{aligned}
$$




$$
\begin{aligned}
& \left\{\begin{array} { l } 
{ U _ { P } ^ { 2 } \triangleq \frac { 1 } { \sqrt { 2 } } ( \Gamma _ { ( \mathfrak { D } - 1 ) ( \mathfrak { D } - 1 ) P } + \Gamma _ { \mathfrak { D } \mathfrak { D } P } ) , } \\
{ V _ { P } ^ { 2 } \triangleq \frac { 1 } { \sqrt { 2 } } ( \Gamma _ { ( \mathfrak { D } - 1 ) ( \mathfrak { D } - 1 ) P } - \Gamma _ { \mathfrak { D } \mathfrak { D } P } ) , }
\end{array} \quad \left\{\begin{array}{l}
X_{P}^{31} \triangleq \frac{1}{\sqrt{2}}\left(\Gamma_{(\mathfrak{D}-1) \mathfrak{D} P}+\Gamma_{\mathfrak{D}(\mathfrak{D}-1) P}\right) \\
Y_{P}^{31} \triangleq \frac{1}{\sqrt{2}}\left(\Gamma_{(\mathfrak{D}-1) \mathfrak{D} P}-\Gamma_{\mathfrak{D}(\mathfrak{D}-1) P}\right)
\end{array}\right.\right. \\
& \left\{\begin{array} { l } 
{ U _ { P } ^ { 3 } \triangleq \frac { 1 } { \sqrt { 2 } } ( \Gamma _ { \mathfrak { D } \mathfrak { D } P } + \Gamma _ { ( \mathfrak { D } - 2 ) ( \mathfrak { D } - 2 ) P } ) , } \\
{ V _ { P } ^ { 3 } \triangleq \frac { 1 } { \sqrt { 2 } } ( \Gamma _ { \mathfrak { D } \mathfrak { D } P } - \Gamma _ { ( \mathfrak { D } - 2 ) ( \mathfrak { D } - 2 ) P } ) , }
\end{array} \quad \left\{\begin{array}{l}
X_{P}^{12} \triangleq \frac{1}{\sqrt{2}}\left(\Gamma_{\mathfrak{D}(\mathfrak{D}-2) P}+\Gamma_{(\mathfrak{D}-2) \mathfrak{D} P}\right), \\
Y_{P}^{12} \triangleq \frac{1}{\sqrt{2}}\left(\Gamma_{\mathfrak{D}(\mathfrak{D}-2) P}-\Gamma_{(\mathfrak{D}-2) \mathfrak{D} P}\right)
\end{array}\right.\right.
\end{aligned}
$$

Definition 7.3. Define the symmetry condition of unification:

(1) Basic conditions, No.1:

$$
\left\{\begin{array}{l}
G^{(\mathfrak{D}-4)(\mathfrak{D}-4)}=G^{(\mathfrak{D}-3)(\mathfrak{D}-3)} \\
G^{(\mathfrak{D}-2)(\mathfrak{D}-2)}=G^{(\mathfrak{D}-1)(\mathfrak{D}-1)}=G^{\mathfrak{D P}}
\end{array}\right.
$$

(2) Basic conditions, No.2:

$$
\left\{\begin{array}{l}
\Gamma_{(\mathfrak{D}-3)(\mathfrak{D}-4) P}=\Gamma_{(\mathfrak{D}-4)(\mathfrak{D}-3) P} \\
\Gamma_{(\mathfrak{D}-2)(\mathfrak{D}-2) P}+\Gamma_{(\mathfrak{D}-1)(\mathfrak{D}-1) P}+\Gamma_{\mathfrak{D} \mathfrak{D} P}=0
\end{array}\right.
$$

(3) MNS mixing conditions of weak interaction, No.1:

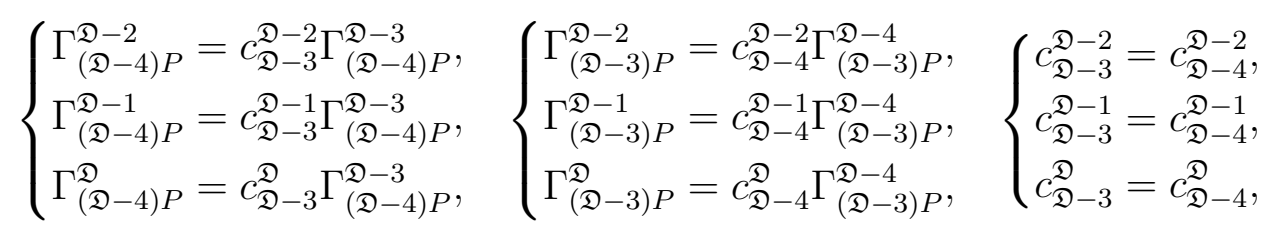

(4) MNS mixing conditions of weak interaction, No.2:

$$
\left\{\begin{array} { l } 
{ \rho _ { ( \mathfrak { D } - 2 ) ( \mathfrak { D } - 3 ) } = \rho _ { ( \mathfrak { D } - 2 ) ( \mathfrak { D } - 4 ) } , } \\
{ \rho _ { ( \mathfrak { D } - 1 ) ( \mathfrak { D } - 3 ) } = \rho _ { ( \mathfrak { D } - 1 ) ( \mathfrak { D } - 4 ) } , } \\
{ \rho _ { \mathfrak { D } ( \mathfrak { D } - 3 ) } = \rho _ { \mathfrak { D } ( \mathfrak { D } - 4 ) } , }
\end{array} \quad \left\{\begin{array}{l}
\rho_{(\mathfrak{D}-3)(\mathfrak{D}-2)}=\rho_{(\mathfrak{D}-4)(\mathfrak{D}-2)} \\
\rho_{(\mathfrak{D}-3)(\mathfrak{D}-1)}=\rho_{(\mathfrak{D}-4)(\mathfrak{D}-1)} \\
\rho_{(\mathfrak{D}-3) \mathfrak{D}}=\rho_{(\mathfrak{D}-4) \mathfrak{D}}
\end{array}\right.\right.
$$

(5) CKM mixing conditions of strong interaction, No.1:

$$
\left\{\begin{array} { l } 
{ \Gamma _ { ( \mathfrak { D } - 2 ) P } ^ { \mathfrak { D } - 3 } = c _ { \mathfrak { D } - 2 } ^ { \mathfrak { D } - 4 } \Gamma _ { ( \mathfrak { D } - 4 ) P } ^ { \mathfrak { D } - 3 } , } \\
{ \Gamma _ { ( \mathfrak { D } - 1 ) P } ^ { \mathfrak { D } - 3 } = c _ { \mathfrak { D } - 1 } ^ { \mathfrak { D } - 4 } \Gamma _ { ( \mathfrak { D } - 4 ) P } ^ { \mathfrak { D } - 3 } , } \\
{ \Gamma _ { \mathfrak { D } P } ^ { \mathfrak { D } - 3 } = c _ { \mathfrak { D } - 4 } ^ { \mathfrak { D } - 4 } \Gamma _ { ( \mathfrak { D } - 4 ) P } ^ { \mathfrak { D } - 3 } , }
\end{array} \quad \left\{\begin{array} { l } 
{ \Gamma _ { ( \mathfrak { D } - 2 ) P } ^ { \mathfrak { D } - 4 } = c _ { \mathfrak { D } - 2 } ^ { \mathfrak { D } - 3 } \Gamma _ { ( \mathfrak { D } - 3 ) P } ^ { \mathfrak { D } - 4 } , } \\
{ \Gamma _ { ( \mathfrak { D } - 1 ) P } ^ { \mathfrak { D } - 4 } = c _ { \mathfrak { D } - 1 } ^ { \mathfrak { D } - 3 } \Gamma _ { ( \mathfrak { D } - 3 ) P } ^ { \mathfrak { D } - 4 } , } \\
{ \Gamma _ { \mathfrak { D } P } ^ { \mathfrak { D } - 4 } = c _ { \mathfrak { D } - 3 } ^ { \mathfrak { D } - 3 } \Gamma _ { ( \mathfrak { D } - 3 ) P } ^ { \mathfrak { D } - 4 } , }
\end{array} \quad \left\{\begin{array}{l}
c_{\mathfrak{D}-2}^{\mathfrak{D}-4}=c_{\mathfrak{D}-1}^{\mathfrak{D}-4}=c_{\mathfrak{D}-4}^{\mathfrak{D}-4} \\
c_{\mathfrak{D}-2}^{\mathfrak{D}-3}=c_{\mathfrak{D}-1}^{\mathfrak{D}-3}=c_{\mathfrak{D}-3}^{\mathfrak{D}-3},
\end{array}\right.\right.\right.
$$

(6) CKM mixing conditions of strong interaction, No.2:

$$
\left\{\begin{array} { l } 
{ \rho _ { ( \mathfrak { D } - 2 ) ( \mathfrak { D } - 3 ) } = \rho _ { ( \mathfrak { D } - 1 ) ( \mathfrak { D } - 3 ) } = \rho _ { \mathfrak { D } ( \mathfrak { D } - 3 ) } , } \\
{ \rho _ { ( \mathfrak { D } - 2 ) ( \mathfrak { D } - 4 ) } = \rho _ { ( \mathfrak { D } - 1 ) ( \mathfrak { D } - 4 ) } = \rho _ { \mathfrak { D } ( \mathfrak { D } - 4 ) } , }
\end{array} \quad \left\{\begin{array}{l}
\rho_{(\mathfrak{D}-3)(\mathfrak{D}-2)}=\rho_{(\mathfrak{D}-3)(\mathfrak{D}-1)}=\rho_{(\mathfrak{D}-3) \mathfrak{D}} \\
\rho_{(\mathfrak{D}-4)(\mathfrak{D}-2)}=\rho_{(\mathfrak{D}-4)(\mathfrak{D}-1)}=\rho_{(\mathfrak{D}-4) \mathfrak{D}}
\end{array}\right.\right.
$$

where $c_{n}^{m}$ are constants. 
Proposition 7.1. When $(M, g)$ satisfies the symmetry conditions of Definition 7.3, denote

$$
\begin{aligned}
& l^{\prime} \triangleq\left(\rho_{(\mathfrak{D}-4)(\mathfrak{D}-4)}+\frac{c_{\mathfrak{D}-4}^{\mathfrak{D}-2}}{2}\left(\rho_{(\mathfrak{D}-2)(\mathfrak{D}-4)}+\rho_{(\mathfrak{D}-4)(\mathfrak{D}-2)}\right)\right. \\
& +\frac{c_{\mathfrak{D}-4}^{\mathfrak{D}-1}}{2}\left(\rho_{(\mathfrak{D}-1)(\mathfrak{D}-4)}+\rho_{(\mathfrak{D}-4)(\mathfrak{D}-1)}\right)+\frac{c_{\mathfrak{D}-4}^{\mathfrak{D}}}{2}\left(\rho_{\mathfrak{D}(\mathfrak{D}-4)}+\rho_{(\mathfrak{D}-4) \mathfrak{D}}\right), \\
& \rho_{(\mathfrak{D}-3)(\mathfrak{D}-3)}+\frac{c_{\mathfrak{D}-3}^{\mathfrak{D}-2}}{2}\left(\rho_{(\mathfrak{D}-2)(\mathfrak{D}-3)}+\rho_{(\mathfrak{D}-3)(\mathfrak{D}-2)}\right) \\
& \left.+\frac{c_{\mathfrak{D}-3}^{\mathfrak{D}-1}}{2}\left(\rho_{(\mathfrak{D}-1)(\mathfrak{D}-3)}+\rho_{(\mathfrak{D}-3)(\mathfrak{D}-1)}\right)+\frac{c_{\mathfrak{D}-3}^{\mathfrak{D}}}{2}\left(\rho_{\mathfrak{D}(\mathfrak{D}-3)}+\rho_{(\mathfrak{D}-3) \mathfrak{D}}\right)\right), \\
& \nu^{\prime} \triangleq\left(\rho_{(\mathfrak{D}-3)(\mathfrak{D}-4)}+\frac{c_{\mathfrak{D}-3}^{\mathfrak{D}-2}}{2}\left(\rho_{(\mathfrak{D}-2)(\mathfrak{D}-4)}+\rho_{(\mathfrak{D}-4)(\mathfrak{D}-2)}\right)\right. \\
& +\frac{c_{\mathfrak{D}-3}^{\mathfrak{D}-1}}{2}\left(\rho_{(\mathfrak{D}-1)(\mathfrak{D}-4)}+\rho_{(\mathfrak{D}-4)(\mathfrak{D}-1)}\right)+\frac{c_{\mathfrak{D}-3}^{\mathfrak{D}}}{2}\left(\rho_{\mathfrak{D}(\mathfrak{D}-4)}+\rho_{(\mathfrak{D}-4) \mathfrak{D}}\right), \\
& \rho_{(\mathfrak{D}-4)(\mathfrak{D}-3)}+\frac{c_{\mathfrak{D}-4}^{\mathfrak{D}-2}}{2}\left(\rho_{(\mathfrak{D}-2)(\mathfrak{D}-3)}+\rho_{(\mathfrak{D}-3)(\mathfrak{D}-2)}\right) \\
& \left.+\frac{c_{\mathfrak{D}-4}^{\mathfrak{D}-1}}{2}\left(\rho_{(\mathfrak{D}-1)(\mathfrak{D}-3)}+\rho_{(\mathfrak{D}-3)(\mathfrak{D}-1)}\right)+\frac{c_{\mathfrak{D}-4}^{\mathfrak{D}}}{2}\left(\rho_{\mathfrak{D}(\mathfrak{D}-3)}+\rho_{(\mathfrak{D}-3) \mathfrak{D}}\right)\right) .
\end{aligned}
$$

Thus, the intrinsic geometric properties $l$ and $\nu$ of $f$ satisfy the following conclusions on $(M, g)$.

$$
\left\{\begin{array}{l}
l_{L ; P}=\partial_{P} l_{L}-g l_{L} Z_{P}-g l_{R} A_{P}-g \nu_{L}^{\prime} W_{P}^{1}, \\
l_{R ; P}=\partial_{P} l_{R}-g l_{R} Z_{P}-g l_{L} A_{P} \\
\nu_{L ; P}=\partial_{P} \nu_{L}-g \nu_{L} Z_{P}-g l_{L}^{\prime} W_{P}^{1} \\
\nu_{R ; P}=\partial_{P} \nu_{R}-g \nu_{R} Z_{P} .
\end{array}\right.
$$

Proof. First, we compute the covariant differential of $\rho_{m n}$ of $f$.

$$
\begin{aligned}
\rho_{m n ; P} & =\partial_{P} \rho_{m n}-\rho_{H n} \Gamma_{m P}^{H}-\rho_{m H} \Gamma_{n P}^{H} \\
& =\partial_{P} \rho_{m n}-\rho_{(\mathfrak{D}-4) n} \Gamma_{m P}^{\mathfrak{D}-4}-\rho_{(\mathfrak{D}-3) n} \Gamma_{m P}^{\mathfrak{D}-3}-\rho_{(\mathfrak{D}-2) n} \Gamma_{m P}^{\mathfrak{D}-2}-\rho_{(\mathfrak{D}-1) n} \Gamma_{m P}^{\mathfrak{D}-1}-\rho_{\mathfrak{D} n} \Gamma_{m P}^{\mathfrak{D}} \\
& -\rho_{m(\mathfrak{D}-4)} \Gamma_{n P}^{\mathfrak{D}-4}-\rho_{m(\mathfrak{D}-3)} \Gamma_{n P}^{\mathfrak{D}-3}-\rho_{m(\mathfrak{D}-2)} \Gamma_{n P}^{\mathfrak{D}-2}-\rho_{m(\mathfrak{D}-1)} \Gamma_{n P}^{\mathfrak{D}-1}-\rho_{m \mathfrak{D}} \Gamma_{n P}^{\mathfrak{D}} .
\end{aligned}
$$

According to Definition 7.2 and Definition 7.3, by calculation we obtain that

$$
\begin{aligned}
l_{L ; P} & =\partial_{P} l_{L}-g l_{L} Z_{P}-g l_{R} A_{P}-g \nu_{L} W_{P}^{1} \\
& -\frac{1}{2}\left[c_{\mathfrak{D}-4}^{\mathfrak{D}-2}\left(\rho_{(\mathfrak{D}-2)(\mathfrak{D}-3)}+\rho_{(\mathfrak{D}-3)(\mathfrak{D}-2)}\right)+c_{\mathfrak{D}-3}^{\mathfrak{D}-2}\left(\rho_{(\mathfrak{D}-2)(\mathfrak{D}-4)}+\rho_{(\mathfrak{D}-4)(\mathfrak{D}-2)}\right)\right] \frac{g}{\sqrt{2}} W_{P}^{1} \\
& -\frac{1}{2}\left[c_{\mathfrak{D}-4}^{\mathfrak{D}-1}\left(\rho_{(\mathfrak{D}-1)(\mathfrak{D}-3)}+\rho_{(\mathfrak{D}-3)(\mathfrak{D}-1)}\right)+c_{\mathfrak{D}-3}^{\mathfrak{D}-1}\left(\rho_{(\mathfrak{D}-1)(\mathfrak{D}-4)}+\rho_{(\mathfrak{D}-4)(\mathfrak{D}-1)}\right)\right] \frac{g}{\sqrt{2}} W_{P}^{1} \\
& -\frac{1}{2}\left[c_{\mathfrak{D}-4}^{\mathfrak{D}}\left(\rho_{\mathfrak{D}(\mathfrak{D}-3)}+\rho_{(\mathfrak{D}-3) \mathfrak{D}}\right)+c_{\mathfrak{D}-3}^{\mathfrak{D}}\left(\rho_{\mathfrak{D}(\mathfrak{D}-4)}+\rho_{(\mathfrak{D}-4) \mathfrak{D}}\right)\right] \frac{g}{\sqrt{2}} W_{P}^{1}, \\
l_{R ; P} & =\partial_{P} l_{R}-g l_{R} Z_{P}-g l_{L} A_{P},
\end{aligned}
$$




$$
\begin{aligned}
\nu_{L ; P} & =\partial_{P} \nu_{L}-g \nu_{L} Z_{P}-g l_{L} W_{P}^{1} \\
& -\frac{1}{2}\left[c_{\mathfrak{D}-4}^{\mathfrak{D}-2}\left(\rho_{(\mathfrak{D}-2)(\mathfrak{D}-4)}+\rho_{(\mathfrak{D}-4)(\mathfrak{D}-2)}\right)+c_{\mathfrak{D}-3}^{\mathfrak{D}-2}\left(\rho_{(\mathfrak{D}-3)(\mathfrak{D}-2)}+\rho_{(\mathfrak{D}-2)(\mathfrak{D}-3)}\right)\right] \frac{g}{\sqrt{2}} W_{P}^{1} \\
& -\frac{1}{2}\left[c_{\mathfrak{D}-4}^{\mathfrak{D}-1}\left(\rho_{(\mathfrak{D}-1)(\mathfrak{D}-4)}+\rho_{(\mathfrak{D}-4)(\mathfrak{D}-1)}\right)+c_{\mathfrak{D}-3}^{\mathfrak{D}-1}\left(\rho_{(\mathfrak{D}-3)(\mathfrak{D}-1)}+\rho_{(\mathfrak{D}-1)(\mathfrak{D}-3)}\right)\right] \frac{g}{\sqrt{2}} W_{P}^{1} \\
& -\frac{1}{2}\left[c_{\mathfrak{D}-4}^{\mathfrak{D}}\left(\rho_{\mathfrak{D}(\mathfrak{D}-4)}+\rho_{(\mathfrak{D}-4) \mathfrak{D}}\right)+c_{\mathfrak{D}-3}^{\mathfrak{D}}\left(\rho_{(\mathfrak{D}-3) \mathfrak{D}}+\rho_{\mathfrak{D}(\mathfrak{D}-3)}\right)\right] \frac{g}{\sqrt{2}} W_{P}^{1}, \\
\nu_{R ; P} & =\partial_{P} \nu_{R}-g \nu_{R} Z_{P} .
\end{aligned}
$$

Then, according to definitions of $l^{\prime}$ and $\nu^{\prime}$, we obtain that

$$
\begin{aligned}
l_{L}^{\prime} & =l_{L} \\
& +\frac{c_{\mathfrak{D}-4}^{\mathfrak{D}-2}}{2 \sqrt{2}}\left(\rho_{(\mathfrak{D}-2)(\mathfrak{D}-4)}+\rho_{(\mathfrak{D}-4)(\mathfrak{D}-2)}\right)+\frac{c_{\mathfrak{D}-4}^{\mathfrak{D}-1}}{2 \sqrt{2}}\left(\rho_{(\mathfrak{D}-1)(\mathfrak{D}-4)}+\rho_{(\mathfrak{D}-4)(\mathfrak{D}-1)}\right)+\frac{c_{\mathfrak{D}-4}^{\mathfrak{D}}}{2 \sqrt{2}}\left(\rho_{\mathfrak{D}(\mathfrak{D}-4)}+\rho_{(\mathfrak{D}-4) \mathfrak{D}}\right) \\
& +\frac{c_{\mathfrak{D}-3}^{\mathfrak{D}-2}}{2 \sqrt{2}}\left(\rho_{(\mathfrak{D}-2)(\mathfrak{D}-3)}+\rho_{(\mathfrak{D}-3)(\mathfrak{D}-2)}\right)+\frac{c_{\mathfrak{D}-3}^{\mathfrak{D}-1}}{2 \sqrt{2}}\left(\rho_{(\mathfrak{D}-1)(\mathfrak{D}-3)}+\rho_{(\mathfrak{D}-3)(\mathfrak{D}-1)}\right)+\frac{c_{\mathfrak{D}-3}^{\mathfrak{D}}}{2 \sqrt{2}}\left(\rho_{\mathfrak{D}(\mathfrak{D}-3)}+\rho_{(\mathfrak{D}-3) \mathfrak{D}}\right) \\
\nu_{L}^{\prime} & =\nu_{L} \\
& +\frac{c_{\mathfrak{D}-3}^{\mathfrak{D}-2}}{2 \sqrt{2}}\left(\rho_{(\mathfrak{D}-2)(\mathfrak{D}-4)}+\rho_{(\mathfrak{D}-4)(\mathfrak{D}-2)}\right)+\frac{c_{\mathfrak{D}-3}^{\mathfrak{D}-1}}{2 \sqrt{2}}\left(\rho_{(\mathfrak{D}-1)(\mathfrak{D}-4)}+\rho_{(\mathfrak{D}-4)(\mathfrak{D}-1)}\right)+\frac{c_{\mathfrak{D}-3}^{\mathfrak{D}}}{2 \sqrt{2}}\left(\rho_{\mathfrak{D}(\mathfrak{D}-4)}+\rho_{(\mathfrak{D}-4) \mathfrak{D}}\right) \\
& +\frac{c_{\mathfrak{D}-4}^{\mathfrak{D}-2}}{2 \sqrt{2}}\left(\rho_{(\mathfrak{D}-2)(\mathfrak{D}-3)}+\rho_{(\mathfrak{D}-3)(\mathfrak{D}-2)}\right)+\frac{c_{\mathfrak{D}-4}^{\mathfrak{D}-1}}{2 \sqrt{2}}\left(\rho_{(\mathfrak{D}-1)(\mathfrak{D}-3)}+\rho_{(\mathfrak{D}-3)(\mathfrak{D}-1)}\right)+\frac{c_{\mathfrak{D}-4}^{\mathfrak{D}}}{2 \sqrt{2}}\left(\rho_{\mathfrak{D}(\mathfrak{D}-3)}+\rho_{(\mathfrak{D}-3) \mathfrak{D}}\right) .
\end{aligned}
$$

Substitute them into the previous equations, and we obtain that

$$
\left\{\begin{array}{l}
l_{L ; P}=\partial_{P} l_{L}-g l_{L} Z_{P}-g l_{R} A_{P}-g \nu_{L}^{\prime} W_{P}^{1}, \\
l_{R ; P}=\partial_{P} l_{R}-g l_{R} Z_{P}-g l_{L} A_{P},
\end{array}, \quad\left\{\begin{array}{l}
\nu_{L ; P}=\partial_{P} \nu_{L}-g \nu_{L} Z_{P}-g l_{L}^{\prime} W_{P}^{1}, \\
\nu_{R ; P}=\partial_{P} \nu_{R}-g \nu_{R} Z_{P} .
\end{array}\right.\right.
$$

Remark 7.1. Reviewing Discussion 5.1, we know the above proposition shows the geometric origin of MNS mixing of weak interaction from the perspective of intrinsic geometry. In affine connection representation of gauge fields, MNS mixing spontaneously appears as an intrinsic geometric property, therefore it is not necessary to be postulated artificially like that in the Standard Model.

In conventional physics, $e, \mu$ and $\tau$ have just only ontological differences, but they have no difference in mathematical connotation. By contrast, Proposition 7.1 tells us that leptons of three generations should be constructed by different linear combinations of $\rho_{(\mathfrak{D}-2)(\mathfrak{D}-3)}, \rho_{(\mathfrak{D}-3)(\mathfrak{D}-2)}$, $\rho_{(\mathfrak{D}-1)(\mathfrak{D}-3)}, \quad \rho_{(\mathfrak{D}-3)(\mathfrak{D}-1)}, \rho_{\mathfrak{D}(\mathfrak{D}-3)}, \quad \rho_{(\mathfrak{D}-3) \mathfrak{D}}, \quad \rho_{(\mathfrak{D}-2)(\mathfrak{D}-4)}, \quad \rho_{(\mathfrak{D}-4)(\mathfrak{D}-2)}, \quad \rho_{(\mathfrak{D}-1)(\mathfrak{D}-4)}, \rho_{(\mathfrak{D}-4)(\mathfrak{D}-1)}$, $\rho_{\mathfrak{D}(\mathfrak{D}-4)}$ and $\rho_{(\mathfrak{D}-4) \mathfrak{D}}$. Thus, $e, \mu$ and $\tau$ may have concrete and distinguishable mathematical connotations. For example, suppose $a_{\tau}, b_{\tau}, a_{\tau_{n}}^{m}, b_{\tau_{n}}^{m}$ are constants, then we can imagine that

$$
\left\{\begin{array}{l}
e \triangleq l=\left(\rho_{(\mathfrak{D}-4)(\mathfrak{D}-4)}, \quad \rho_{(\mathfrak{D}-3)(\mathfrak{D}-3)}\right) \\
\nu_{e} \triangleq \nu=\left(\rho_{(\mathfrak{D}-3)(\mathfrak{D}-4)}, \quad \rho_{(\mathfrak{D}-4)(\mathfrak{D}-3)}\right) .
\end{array}\right.
$$




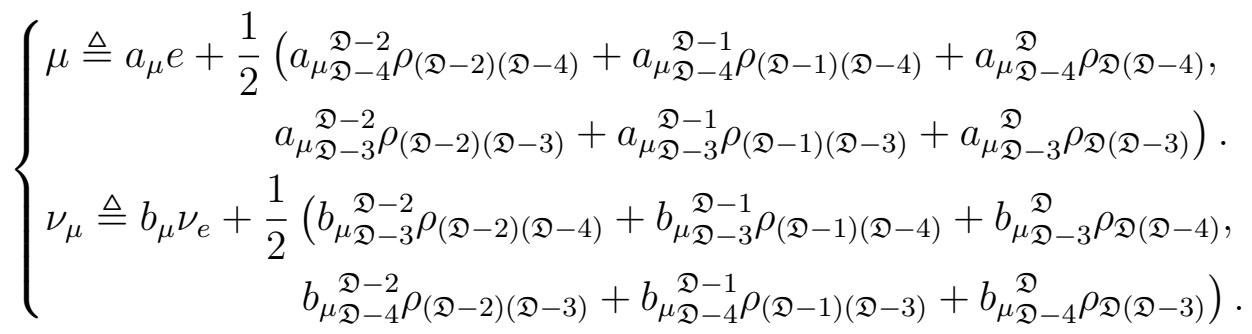

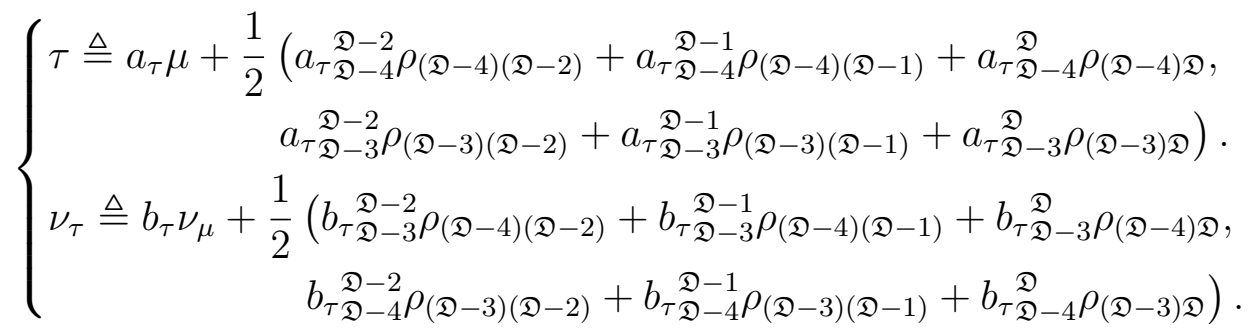

Proposition 7.2. When $(M, g)$ satisfies the symmetry conditions of Definition 7.3, denote

$$
\begin{aligned}
& d_{1 L}^{\prime} \triangleq \frac{1}{2 \sqrt{2}} c_{\mathfrak{D}-1}^{\mathfrak{D}-3}\left(\rho_{(\mathfrak{D}-4)(\mathfrak{D}-2)}+\rho_{(\mathfrak{D}-2)(\mathfrak{D}-4)}\right)+\frac{1}{2 \sqrt{2}} c_{\mathfrak{D}-2}^{\mathfrak{D}-3}\left(\rho_{(\mathfrak{D}-4)(\mathfrak{D}-1)}+\rho_{(\mathfrak{D}-1)(\mathfrak{D}-4)}\right) \\
& +\frac{1}{2 \sqrt{2}} c_{\mathfrak{D}-1}^{\mathfrak{D}-4}\left(\rho_{(\mathfrak{D}-3)(\mathfrak{D}-2)}+\rho_{(\mathfrak{D}-2)(\mathfrak{D}-3)}\right)+\frac{1}{2 \sqrt{2}} c_{\mathfrak{D}-2}^{\mathfrak{D}-4}\left(\rho_{(\mathfrak{D}-3)(\mathfrak{D}-1)}+\rho_{(\mathfrak{D}-1)(\mathfrak{D}-3)}\right), \\
& d_{2 L}^{\prime} \triangleq \frac{1}{2 \sqrt{2}} c_{\mathfrak{D}}^{\mathfrak{D}-3}\left(\rho_{(\mathfrak{D}-4)(\mathfrak{D}-1)}+\rho_{(\mathfrak{D}-1)(\mathfrak{D}-4)}\right)+\frac{1}{2 \sqrt{2}} c_{\mathfrak{D}-1}^{\mathfrak{D}-3}\left(\rho_{(\mathfrak{D}-4) \mathfrak{D}}+\rho_{\mathfrak{D}(\mathfrak{D}-4)}\right) \\
& +\frac{1}{2 \sqrt{2}} c_{\mathfrak{D}}^{\mathfrak{D}-4}\left(\rho_{(\mathfrak{D}-3)(\mathfrak{D}-1)}+\rho_{(\mathfrak{D}-1)(\mathfrak{D}-3)}\right)+\frac{1}{2 \sqrt{2}} c_{\mathfrak{D}-1}^{\mathfrak{D}-4}\left(\rho_{(\mathfrak{D}-3) \mathfrak{D}}+\rho_{\mathfrak{D}(\mathfrak{D}-3)}\right), \\
& d_{3 L}^{\prime} \triangleq \frac{1}{2 \sqrt{2}} c_{\mathfrak{D}-2}^{\mathfrak{D}-3}\left(\rho_{(\mathfrak{D}-4) \mathfrak{D}}+\rho_{\mathfrak{D}(\mathfrak{D}-4)}\right)+\frac{1}{2 \sqrt{2}} c_{\mathfrak{D}}^{\mathfrak{D}-3}\left(\rho_{(\mathfrak{D}-4)(\mathfrak{D}-2)}+\rho_{(\mathfrak{D}-2)(\mathfrak{D}-4)}\right) \\
& +\frac{1}{2 \sqrt{2}} c_{\mathfrak{D}-2}^{\mathfrak{D}-4}\left(\rho_{(\mathfrak{D}-3) \mathfrak{D}}+\rho_{\mathfrak{D}(\mathfrak{D}-3)}\right)+\frac{1}{2 \sqrt{2}} c_{\mathfrak{D}-4}^{\mathfrak{D}}\left(\rho_{(\mathfrak{D}-3)(\mathfrak{D}-2)}+\rho_{(\mathfrak{D}-2)(\mathfrak{D}-3)}\right), \\
& u_{1 L}^{\prime} \triangleq \frac{1}{2 \sqrt{2}} c_{\mathfrak{D}-2}^{\mathfrak{D}-3}\left(\rho_{(\mathfrak{D}-4)(\mathfrak{D}-2)}+\rho_{(\mathfrak{D}-2)(\mathfrak{D}-4)}\right)+\frac{1}{2 \sqrt{2}} c_{\mathfrak{D}-2}^{\mathfrak{D}-4}\left(\rho_{(\mathfrak{D}-3)(\mathfrak{D}-2)}+\rho_{(\mathfrak{D}-2)(\mathfrak{D}-3)}\right) \\
& +\frac{1}{2 \sqrt{2}} c_{\mathfrak{D}-1}^{\mathfrak{D}-3}\left(\rho_{(\mathfrak{D}-4)(\mathfrak{D}-1)}+\rho_{(\mathfrak{D}-1)(\mathfrak{D}-4)}\right)+\frac{1}{2 \sqrt{2}} c_{\mathfrak{D}-1}^{\mathfrak{D}-4}\left(\rho_{(\mathfrak{D}-3)(\mathfrak{D}-1)}+\rho_{(\mathfrak{D}-1)(\mathfrak{D}-3)}\right), \\
& u_{2 L}^{\prime} \triangleq \frac{1}{2 \sqrt{2}} c_{\mathfrak{D}-1}^{\mathfrak{D}-3}\left(\rho_{(\mathfrak{D}-4)(\mathfrak{D}-1)}+\rho_{(\mathfrak{D}-1)(\mathfrak{D}-4)}\right)+\frac{1}{2 \sqrt{2}} c_{\mathfrak{D}-1}^{\mathfrak{D}-4}\left(\rho_{(\mathfrak{D}-3)(\mathfrak{D}-1)}+\rho_{(\mathfrak{D}-1)(\mathfrak{D}-3)}\right) \\
& +\frac{1}{2 \sqrt{2}} c_{\mathfrak{D}}^{\mathfrak{D}-3}\left(\rho_{(\mathfrak{D}-4) \mathfrak{D}}+\rho_{\mathfrak{D}(\mathfrak{D}-4)}\right)+\frac{1}{2 \sqrt{2}} c_{\mathfrak{D}}^{\mathfrak{D}-4}\left(\rho_{(\mathfrak{D}-3) \mathfrak{D}}+\rho_{\mathfrak{D}(\mathfrak{D}-3)}\right), \\
& u_{3 L}^{\prime} \triangleq \frac{1}{2 \sqrt{2}} c_{\mathfrak{D}}^{\mathfrak{D}-3}\left(\rho_{(\mathfrak{D}-4) \mathfrak{D}}+\rho_{\mathfrak{D}(\mathfrak{D}-4)}\right)+\frac{1}{2 \sqrt{2}} c_{\mathfrak{D}}^{\mathfrak{D}-4}\left(\rho_{(\mathfrak{D}-3) \mathfrak{D}}+\rho_{\mathfrak{D}(\mathfrak{D}-3)}\right) \\
& +\frac{1}{2 \sqrt{2}} c_{\mathfrak{D}-2}^{\mathfrak{D}-3}\left(\rho_{(\mathfrak{D}-4)(\mathfrak{D}-2)}+\rho_{(\mathfrak{D}-2)(\mathfrak{D}-4)}\right)+\frac{1}{2 \sqrt{2}} c_{\mathfrak{D}-2}^{\mathfrak{D}-4}\left(\rho_{(\mathfrak{D}-3)(\mathfrak{D}-2)}+\rho_{(\mathfrak{D}-2)(\mathfrak{D}-3)}\right) .
\end{aligned}
$$

Then the intrinsic geometric properties $d_{1}, d_{2}, d_{3}, u_{1}, u_{2}, u_{3}$ of $f$ satisfy the following conclusions on $(M, g)$. 


$$
\begin{aligned}
& d_{1 L ; P}=\partial_{P} d_{1 L}-g_{s} d_{1 L} U_{P}^{1}+g_{s} d_{2 L} V_{P}^{1}-g_{s} d_{3 L} V_{P}^{1} \\
& -g_{s} u_{1 L} X_{P}^{23}-\frac{g_{s}}{2} u_{2 L} X_{P}^{31}+\frac{g_{s}}{2} u_{2 L} Y_{P}^{31}-\frac{g_{s}}{2} u_{3 L} X_{P}^{12}-\frac{g_{s}}{2} u_{3 L} Y_{P}^{12}-g u_{1 L}^{\prime} W_{P}^{1}, \\
& d_{2 L ; P}=\partial_{P} d_{2 L}-g_{s} d_{2 L} U_{P}^{2}+g_{s} d_{3 L} V_{P}^{2}-g_{s} d_{1 L} V_{P}^{2} \\
& -g_{s} u_{2 L} X_{P}^{31}-\frac{g_{s}}{2} u_{3 L} X_{P}^{12}+\frac{g_{s}}{2} u_{3 L} Y_{P}^{12}-\frac{g_{s}}{2} u_{1 L} X_{P}^{23}-\frac{g_{s}}{2} u_{1 L} Y_{P}^{23}-g u_{2 L}^{\prime} W_{P}^{1}, \\
& d_{3 L ; P}=\partial_{P} d_{3 L}-g_{s} d_{3 L} U_{P}^{3}+g_{s} d_{1 L} V_{P}^{3}-g_{s} d_{2 L} V_{P}^{3} \\
& -g_{s} u_{3 L} X_{P}^{12}-\frac{g_{s}}{2} u_{1 L} X_{P}^{23}+\frac{g_{s}}{2} u_{1 L} Y_{P}^{23}-\frac{g_{s}}{2} u_{2 L} X_{P}^{31}-\frac{g_{s}}{2} u_{2 L} Y_{P}^{31}-g u_{3 L}^{\prime} W_{P}^{1}, \\
& d_{1 R ; P}=\partial_{P} d_{1 R}-g_{s} d_{1 L} V_{P}^{1}+g_{s} d_{2 L} U_{P}^{1}-g_{s} d_{3 L} U_{P}^{1} \\
& +g_{s} u_{1 L} Y_{P}^{23}+\frac{g_{s}}{2} u_{2 L} X_{P}^{31}-\frac{g_{s}}{2} u_{2 L} Y_{P}^{31}-\frac{g_{s}}{2} u_{3 L} X_{P}^{12}-\frac{g_{s}}{2} u_{3 L} Y_{P}^{12}, \\
& d_{2 R ; P}=\partial_{P} d_{2 R}-g_{s} d_{2 L} V_{P}^{2}+g_{s} d_{3 L} U_{P}^{2}-g_{s} d_{1 L} U_{P}^{2} \\
& +g_{s} u_{2 L} Y_{P}^{31}+\frac{g_{s}}{2} u_{3 L} X_{P}^{12}-\frac{g_{s}}{2} u_{3 L} Y_{P}^{12}-\frac{g_{s}}{2} u_{1 L} X_{P}^{23}-\frac{g_{s}}{2} u_{1 L} Y_{P}^{23}, \\
& d_{3 R ; P}=\partial_{P} d_{3 R}-g_{s} d_{3 L} V_{P}^{3}+g_{s} d_{1 L} U_{P}^{3}-g_{s} d_{2 L} U_{P}^{3} \\
& +g_{s} u_{3 L} Y_{P}^{12}+\frac{g_{s}}{2} u_{1 L} X_{P}^{23}-\frac{g_{s}}{2} u_{1 L} Y_{P}^{23}-\frac{g_{s}}{2} u_{2 L} X_{P}^{31}-\frac{g_{s}}{2} u_{2 L} Y_{P}^{31}, \\
& u_{1 L ; P}=\partial_{P} u_{1 L}-g_{s} u_{1 L} U_{P}^{1}-\frac{g_{s}}{2} u_{2 L} X_{P}^{12}-\frac{g_{s}}{2} u_{2 L} Y_{P}^{12}-\frac{g_{s}}{2} u_{3 L} X_{P}^{31}+\frac{g_{s}}{2} u_{3 L} Y_{P}^{31} \\
& -g_{s} d_{1 L} X_{P}^{23}+g_{s} d_{2 L} Y_{P}^{23}-g_{s} d_{3 L} Y_{P}^{23}-g d_{1 L}^{\prime} W_{P}^{1}, \\
& u_{2 L ; P}=\partial_{P} u_{2 L}-g_{s} u_{2 L} U_{P}^{2}-\frac{g_{s}}{2} u_{3 L} X_{P}^{23}-\frac{g_{s}}{2} u_{3 L} Y_{P}^{23}-\frac{g_{s}}{2} u_{1 L} X_{P}^{12}+\frac{g_{s}}{2} u_{1 L} Y_{P}^{12} \\
& -g_{s} d_{2 L} X_{P}^{31}+g_{s} d_{3 L} Y_{P}^{31}-g_{s} d_{1 L} Y_{P}^{31}-g d_{2 L}^{\prime} W_{P}^{1}, \\
& u_{3 L ; P}=\partial_{P} u_{3 L}-g_{s} u_{3 L} U_{P}^{3}-\frac{g_{s}}{2} u_{1 L} X_{P}^{31}-\frac{g_{s}}{2} u_{1 L} Y_{P}^{31}-\frac{g_{s}}{2} u_{2 L} X_{P}^{23}+\frac{g_{s}}{2} u_{2 L} Y_{P}^{23} \\
& -g_{s} d_{3 L} X_{P}^{12}+g_{s} d_{1 L} Y_{P}^{12}-g_{s} d_{2 L} Y_{P}^{12}-g d_{3 L}^{\prime} W_{P}^{1}, \\
& u_{1 R ; P}=\partial_{P} u_{1 R}-g_{s} u_{1 R} U_{P}^{1}+\frac{g_{s}}{2} u_{2 R} X_{P}^{12}+\frac{g_{s}}{2} u_{2 R} Y_{P}^{12}+\frac{g_{s}}{2} u_{3 R} X_{P}^{31}-\frac{g_{s}}{2} u_{3 R} Y_{P}^{31} \text {, } \\
& u_{2 R ; P}=\partial_{P} u_{2 R}-g_{s} u_{2 R} U_{P}^{2}+\frac{g_{s}}{2} u_{3 R} X_{P}^{23}+\frac{g_{s}}{2} u_{3 R} Y_{P}^{23}+\frac{g_{s}}{2} u_{1 R} X_{P}^{12}-\frac{g_{s}}{2} u_{1 R} Y_{P}^{12}, \\
& u_{3 R ; P}=\partial_{P} u_{3 R}-g_{s} u_{3 R} U_{P}^{3}+\frac{g_{s}}{2} u_{1 R} X_{P}^{31}+\frac{g_{s}}{2} u_{1 R} Y_{P}^{31}+\frac{g_{s}}{2} u_{2 R} X_{P}^{23}-\frac{g_{s}}{2} u_{2 R} Y_{P}^{23} .
\end{aligned}
$$

Proof. Substitute Definition 7.2 into $\rho_{m n}$ and consider Definition 7.3, then compute them, and then substitute $d_{1 L}^{\prime}, d_{2 L}^{\prime}, d_{3 L}^{\prime}, u_{1 L}^{\prime}, u_{2 L}^{\prime}, u_{3 L}^{\prime}$ into them, we finally obtain the results.

Remark 7.2. The above proposition shows the geometric origin of CKM mixing. We see that, in affine connection representation of gauge fields, $d_{1 L}^{\prime}, d_{2 L}^{\prime}, d_{3 L}^{\prime}, u_{1 L}^{\prime}, u_{2 L}^{\prime}, u_{3 L}^{\prime}$ spontaneously appear as intrinsic geometric properties.

In addition, this proposition also shows that the new framework has an advantage that it never causes the problem that a proton decays into a lepton like some GUTs do.

Definition 7.4. If reference-system $f$ satisfies $\rho_{(\mathfrak{D}-2)(\mathfrak{D}-2)}=\rho_{(\mathfrak{D}-1)(\mathfrak{D}-1)}=\rho_{\mathfrak{D} \mathfrak{D}}=\rho_{(\mathfrak{D}-2)(\mathfrak{D}-1)}=$ $\rho_{(\mathfrak{D}-1)(\mathfrak{D}-2)}=\rho_{(\mathfrak{D}-1) \mathfrak{D}}=\rho_{\mathfrak{D}(\mathfrak{D}-1)}=\rho_{\mathfrak{D}(\mathfrak{D}-2)}=\rho_{(\mathfrak{D}-2) \mathfrak{D}}=0$, we say $f$ is a lepton field, otherwise $f$ is a hadron field. 
Suppose $f$ is a hadron field. For $d_{1}, d_{2}, d_{3}, u_{1}, u_{2}, u_{3}$, if $f$ satisfies that five of them are zero and the other one is non-zero, we say $f$ is an individual quark.

Proposition 7.3. There does not exist an individual quark. In other words, if any five ones of $d_{1}, d_{2}, d_{3}, u_{1}, u_{2}, u_{3}$ are zero, then $d_{1}=d_{2}=d_{3}=u_{1}=u_{2}=u_{3}=0$.

Remark 7.3. For an individual down-type quark, the above proposition is evident. Without loss of generality let $u_{1}=u_{2}=u_{3}=0$ and $d_{1}=d_{2}=0$, thus $\rho_{(\mathfrak{D}-2)(\mathfrak{D}-2)}=\rho_{(\mathfrak{D}-1)(\mathfrak{D}-1)}=\rho_{\mathfrak{D D}}=0$, hence we must have $d_{3}=0$.

For an individual up-type quark, this paper has not made progress on the proof yet. Anyway, it is significant that Proposition 7.3 shows the geometric connotation of the color confinement. It involves a natural geometric constraint of the curvatures among different dimensions.

Remark 7.4. On one hand, all of the above discussions in sections 5, 6 and 7 are based on the (1) of Definition 4.6.1. The weak and electromagnetic unified field in Definition 5.1, the strong interaction field in Definition 6.1 and the typical unified gauge field in Definition 7.1 are all special cases of the (1) of Definition 4.6.1. They do not contain gravitational effects.

On the other hand, For the cases containing the gravitational effects, we just need to take the (2) of Definition 4.6.1 or a general reference-system defined in Definition 2.1.1.

\section{Conclusions}

1. An affine connection representation of gauge fields is established in this paper. It has the following main points of view.

(i) Geometric manifold in Definition 2.1.1 is more suitable than Riemannian manifold for describing physical space.

(ii) Simple connection in Definition 2.5.1 contains more geometric information than Levi-Civita connection, and is independent of Christoffel symbol. It can uniformly describe gauge field and gravitational field.

(iii) Time is the total metric with respect to all dimensions of internal coordinate space and external coordinate space.

(iv) On-shell evolution is the evolution along gradient direction.

(v) Quantum evolution is the evolution along distribution of gradient directions. It has a geometric meaning discussed in section 3.10.

2. In the affine connection representation of gauge fields, the following unified mathematical descriptions are achieved.

(i) Gauge field and gravitational field have a unified coordinate description. They can be represented by the same affine connection. The components of $\Gamma_{N P}^{M}$ with $M, N \in\{4,5, \cdots, \mathfrak{D}\}$ describe electromagnetic, weak and strong interaction fields. The other components of $\Gamma_{N P}^{M}$ describe gravitational field.

(ii) Gauge field and elementary particle field have a unified geometric construction. They are both geometric entities constructed with semi-metric.

(iii) Physical evolutions of gauge field and elementary particle field have a unified geometric description. Their on-shell evolution and quantum evolution both present as geometric properties about gradient direction. 
(iv) Quantum theory and gravitational theory have a unified geometric interpretation and the same view of time and space. They both reflect intrinsic geometric properties of manifold.

(v) Several postulates in Standard Model are turned into geometric results that appears spontaneously in affine connection representation, so they are not necessary to be regarded as postulates anymore.

\section{References}

[1] T. W. B. Kibble, Lorentz invariance and the gravitational field, Journal of Mathematical Physics 2 (2) (1961) 212-221. doi : 10.1063/1.1703702.

[2] D. Sciama, Recent developments in general relativity, Festschrift for Infeld, Pergamon, Oxford 415 (1962).

[3] J. P. Harnard, R. B. Pettitt, Gauge theories for space-time symmetries. i, Journal of Mathematical Physics 17 (10) (1976) 1827-1837. doi : 10.1063/1.522829.

[4] P. v. d. Heyde, The field equations of the poincare gauge theory of gravitation, Physics Letters A 58 (3) (1976) 141-143. doi : 10.1016/0375-9601(76)90266-8.

[5] Y. M. Cho, Gauge theory, gravitation, and symmetry, Physical Review D 14:12 (12) (1976) 3341-3344. doi : 10.1103/PhysRevD. 14.3341.

[6] F. W. Hehl, G. D. Kerlick, J. M. Nester, General relativity with spin and torsion: Foundations and prospects, Reviews of Modern Physics 48 (3) (1976) 393-416. doi : 10.1103/ RevModPhys . 48.393.

[7] S. W. Macdowell, F. Mansouri, Unified geometric theory of gravity and supergravity, Physical Review Letters 38 (23) (1977) 739-742. doi : 10.1103/PhysRevLett. 38 . 1376.

[8] J. L. Chkareuli, On the origin of poincare gauge gravity, Physics Letters B 769 (2017) 377-384. doi : 10.1016/j.physletb.2017.04.012.

[9] J. L. Chkareuli, Poincare gauge gravity an emergent scenario, Physical Review D 95 (8) (2017) 084051. doi:10.1103/PhysRevD.95.084051.

[10] Y. N. Obukhov, Poincare gauge gravity: An overview, International Journal of Geometric Methods in Modern Physics 15 (supp01) (2018) 1840005-342. doi:10.1142/ S0219887818400054.

[11] Y. N. Obukhov, F. W. Hehl, General relativity as a special case of poincare gauge gravity, Physical Review D 102 (4) (2020) 044058. doi : 10.1103/PhysRevD . 102 .044058.

[12] F. W. Hehl, G. D. Kerlick, P. v. d. Heyde, On hypermomentum in general relativity i. the notion of hypermomentum, Ztschrift Fur Naturforschung A 31 (2) (1976). doi : 10.1515/ zna-1976-0201.

[13] F. W. Hehl, G. D. Kerlick, P. v. d. Heyde, On hypermomentum in general relativity. ii. the geometry of space-time, Zeitschrift Fur Naturforschung A 31 (6) (1976) 524-527. doi: 10.1515/zna-1976-0602. 
[14] F. W. Hehl, G. David, Kerlick, P. v. d. Heyde, On hypermomentum in general relativity iii. coupling hypermomentum to geometry, Zeitschrift Fur Naturforschung A 31 (7) (1976) 823-827. doi: 10.1515/zna-1976-0724.

[15] F. W. Hehl, On a new metric affine theory of gravitation, Physics Letters B 63 (4) (1976) 446-448. doi : 10.1016/0370-2693(76)90393-2.

[16] F. W. Hehl, E. A. Lord, Y. Ne'Eman, Hadron dilation, shear and spin as components of the intrinsic hypermomentum current and metric-affine theory of gravitation, Physics Letters B 71 (2) (1977) 432-434. doi : 10.1016/0370-2693(77)90260-X.

[17] E. A. Lord, The metric-affine gravitational theory as the gauge theory of the affine group, Physics Letters A 65 (1) (1978) 1-4. doi : 10. 1016/0375-9601(78)90113-5.

[18] F. W. Hehl, J. D. Mccrea, E. W. Mielke, Y. Ne'Eman, Metric-affine gauge theory of gravity: field equations, noether identities, world spinors, and breaking of dilation invariance, Physics Reports-review Section of Physics Letters 258 (1995). doi:10.1016/0370-1573(94) Q0111-F.

[19] R. F. Sobreiro, V. J. V. Otoya, Affine gauge theory of gravity and its reduction to the riemanncartan geometry, Journal of Physics Conference 283 (1) (2010). doi : 10 . 1088/1742-6596/ $283 / 1 / 012032$.

[20] R. F. Sobreiro, V. J. V. Otoya, On the topological reduction from the affine to the orthogonal gauge theory of gravity, Journal of Geometry and Physics 61 (1) (2011) 137-150. doi: $10.1016 / j$. geomphys.2010.09.009.

[21] D. J. Cirilo-Lombardo, Unified field theoretical models from generalized affine geometries, International Journal of Theoretical Physics 49 (6) (2010) 1288-1301. doi:10.1007/ s10773-010-0310-9.

[22] D. J. Cirilo-Lombardo, Unified field theoretical models from generalized affine geometries ii, International Journal of Theoretical Physics 50 (6) (2011) 1699-1708. doi:10.1007/ s10773-011-0678-1.

[23] D. J. Cirilo-Lombardo, Unified field theoretical models from generalized affine geometries iii, International Journal of Theoretical Physics 50 (11) (2011) 3621-3634. doi:10.1007/ s10773-011-0871-2.

[24] L. P. Eisenhart, Riemannian Geometry, Princeton university press, 1926.

[25] Y.-S. Duan, J.-Y. Zhang, Conservation law of energy and momentum in general relativity, Acta physica sinica 19 (11) (1963) 689-704. doi : 10.7498/aps . 19.689.

[26] C. N. Yang, R. L. Mills, Conservation of isotopic spin and isotopic gauge invariance, Physical review 96 (1) (1954) 191. doi : 10.1103/PhysRev.96.191.

[27] S. L. Glashow, Partial-symmetries of weak interactions, Nuclear Physics 22 (4) (1961) 579588. doi : 10.1016/0029-5582(61) 90469-2. 
[28] S. Weinberg, A model of leptons, Physical Review Letters 19 (21) (1967) 1264-1266. doi : 10.1103/PhysRevLett.19.1264.

[29] H. D. Politzer, Asymptotic freedom: An approach to strong interactions, Physics Reports 14 (4) (1974) 129-180. doi : 10.1016/0370-1573(74)90014-3.

[30] P. W. Higgs, Broken symmetries, massless particles and gauge fields, Physics Letters 12 (2) (1964) 132-133. doi : 10.1016/0031-9163(64)91136-9.

[31] P. W. Higgs, Broken symmetries and the masses of gauge bosons, Physical Review Letters 13 (16) (1964) 508-509. doi : 10.1103/PhysRevLett.13.508.

[32] P. W. Higgs, Spontaneous symmetry breakdown without massless bosons, Physical Review 145 (4) (1966) 1156-1163. doi : 10.1103/PhysRev. 145.1156.

[33] F. Englert, R. Brout, Broken symmetry and the mass of gauge vector mesons, Physical Review Letters 13 (9) (1964) 321-323. doi : 10.1103/PhysRevLett. 13 . 321.

[34] G. Guralnik, C. R. Hagen, T. W. B. Kibble, Global conservation laws and massless particles, Physical Review Letters 13 (20) (1964) 585-587. doi : 10.1103/PhysRevLett. 13. 585.

[35] T. W. B. Kibble, Symmetry breaking in non-abelian gauge theories, Physical Review 155 (5) (1967) 1554-1561. doi:10.1103/PhysRev.155.1554.

[36] M. Gell-Mann, Symmetries of baryons and mesons, Physical Review 125 (3) (1962) 1067 1084. doi: 10.1103/PhysRev. 125.1067.

[37] M. Gell-Mann, A schematic model of baryons and mesons, Physics Letters 8 (3) (1964) 214-215. doi : 10.1016/S0031-9163(64)92001-3.

[38] J. D. Bjorken, Asymptotic sum rules at infinite momentum, Physical Review 179 (5) (1969) 1547-1553. doi:10.1103/PhysRev.179.1547.

[39] R. Feynman, Very high-energy collisions of hadrons, Physical Review Letters 23 (24) (1969) 1415-1417. doi: 10.1103/physrevlett.23.1415.

[40] D. J. Gross, F. Wilczek, Ultraviolet behavior of non-abelian gauge theories, Physical Review Letters 30 (26) (1973) 1343-1346. doi : 10.1103/physrevlett.30.1343.

[41] H. D. Politzer, Reliable perturbative results for strong interactions?, Physical Review Letters 30 (26) (1973) 1346-1349. doi : 10.1103/physrevlett.30.1346.

[42] D. J. Gross, F. Wilczek, Asymptotically free gauge theories. i, Physical Review D 8 (10) (1973) 3633-3652. doi:10.1103/PhysRevD. 8.3633.

[43] D. J. Gross, F. Wilczek, Asymptotically free gauge theories. ii, Physical Review D 9 (4) (1973) 980-993. doi : 10.1103/PhysRevD.9.980.

[44] H. Georgi, H. D. Politzer, Electroproduction scaling in an asymptotically free theory of strong interactions, Physical Review D 9 (2) (1974) 416-420. doi : 10 . 1103/PhysRevD . 9. 416. 
[45] Y. Watanabe, L. N. Hand, S. Herb, et al., Test of scale invariance in ratios of muon scattering cross sections at 150 and 56 gev, Physical Review Letters 35 (14) (1975) 898-901. doi: 10.1103/PhysRevLett. 35.898 .

[46] C. Chang, K. W. Chen, D. J. Fox, et al., Observed deviations from scale invariance in highenergy muon scattering, Physical Review Letters 35 (14) (1975) 901-904. doi : 10.1103/ PhysRevLett. 35.901.

[47] H. Georgi, S. L. Glashow, Unity of all elementary-particle force, Physical Review Letters 32 (8) (1974) 438-441. doi : 10.1103/PhysRevLett. 32 . 438.

[48] H. Fritzsch, P. Minkowski, Unified interactions of leptons and hadrons, Annals of Physics 93 (1-2) (1975) 193-266. doi : 10.1016/0003-4916(75)90211-0.

[49] Z. Q. Ma, D. S. Du, P. Y. Xue, et al., A possible su(7) grand unified theory with four generations of light fermions, Scientia Sinica VXXIV (10) (1981) 1358-1365.

[50] Z. Q. Ma, T. S. Tu, P. Y. Xue, et al., An su(8) grand unified model accommodating three generations with v - a coupling, Physics Letters B 100 (5) (1981) 399-402. doi : 10.1016/ 0370-2693(81) 90145-3.

[51] F. X. Dong, D. S. Du, P. Y. Xue, et al., Fractionally charged color singlet fermions and low mass magnetic monopoles in su(9) grand unified models, Scientia Sinica VXXVI (11) (1983) 1189-1197.

[52] Z. Q. Ma, T. S. Tu, X. J. Zhou, et al., Problem of generations and the gut of so(14), High Energy Physics and Nuclear Physics 5 (6) (1981) 664-681.

[53] R. Percacci, Spontaneous soldering, Physics Letters B 144 (1) (1984) 37-40. doi : 10.1016 / 0370-2693 (84)90171-0.

[54] F. Nesti, R. Percacci, Chirality in unified theories of gravity, Physical Review D 81 (2) (2010) 025010. doi : 10.1103/PhysRevD.81.025010.

[55] Y. L. Wu, Theory of quantum gravity beyond einstein and space-time dynamics with quantum inflation, International Journal of Modern Physics A 30 (28n29) (2015) 1545002-1-36. doi : 10. $1142 /$ S0217751X15450025.

[56] Y. L. Wu, Quantum field theory of gravity with spin and scaling gauge invariance and spacetime dynamics with quantum inflation, Physical Review D 93 (2) (2016) 024012-1-35. doi: 10.1103/PhysRevD. 93.024012 .

[57] Y. L. Wu, Maximal symmetry and mass generation of dirac fermions and gravitational gauge field theory in six-dimensional spacetime, Chinese Physics C 41 (10) (2017) 61-73. doi : $10.1088 / 1674-1137 / 41 / 10 / 103106$.

[58] Y. L. Wu, Unified field theory of basic forces and elementary particles with gravitational origin of gauge symmetry in hyper-spacetime, Science Bulletin 62 (16) (2017) 1109-1113. doi : 10.1016/j.scib.2017.08.005. 
[59] Y. L. Wu, Hyperunified field theory and gravitational gaugegeometry duality, The European Physical Journal C 78 (1) (2018) 28. doi : 10.1140/epjc/s10052-017-5504-3.

[60] T. Kaluza, Zum unitätsproblem der physik, Sitzungsber Preuss Akad Wiss Berlin(Math. Phys.) (1921) 966-972doi : 10.1142/S0218271818700017.

[61] O. Klein, Quantentheorie und fünfdimensionale relativitätstheorie, Zeitschrift für Physik A 37 (12) (1926) 895-906. doi : 10.1080/01422418608228771.

[62] O. Klein, The atomicity of electricity as a quantum theory law, Nature 118 (1926) 516. doi : 10.1142/97898145716160007.

[63] P. Goddard, J. Goldstone, C. Rebbi, et al., Quantum dynamics of a massless relativistic string, Nuclear Physics 56 (1) (1973) 109-135. doi : 10. 1016/0550-3213 (73) 90223-X.

[64] J. Polchinski, S. Chaudhuri, C. V. Johnson, Notes on d-branes, arXiv:hep-th/9602052v2 (1996).

[65] W. Taylor, Lectures on d-branes, gauge theory and m(atrices), arXiv:hep-th/9801182v2 (1998).

[66] C. P. Bachas, Lectures on d-branes, arXiv:hep-th/9806199v2 (1999).

[67] A. Giveon, D. Kutasov, Brane dynamics and gauge theory, Review of Modern Physics 71 (4) (1998) 310-318. doi : 10.1103/RevModPhys.71.983.

[68] J. Wess, B. Zumino, Supergauge invariant extension of quantum electrodynamics, Nuclear Physics B 78 (1) (1974) 1-13. doi : 10. 1016/0550-3213(74)90112-6.

[69] A. Hanany, E. Witten, Type iib superstrings, bps monopoles, and three-dimensional gauge dynamics, Nuclear Physics B 492 (1-2) (1997) 152-190. doi : 10. 1016/S0550-3213(97) Q0157-0.

[70] M. R. Douglas, S. Govindarajan, T. Jayaraman, et al., D-branes on calabiyau manifolds and superpotentials, Communications in Mathematical Physics 248 (1) (2004) 85-118. doi : $10.1007 / \mathrm{s} 00220-004-1091-\mathrm{x}$.

[71] H. E. Haber, G. L. Kane, The search for supersymmetry: Probing physics beyond the standard model, Physics Reports 117 (2-4) (1985) 75-263. doi : 10 . 1016/0370-1573 (85) 90051-1.

[72] J. F. Gunion, H. E. Haber, Higgs bosons in supersymmetric models (i), Nuclear Physics B, Particle Physics 272 (1) (1986) 1-76. doi : 10.1016/0550-3213(86)90340-8.

[73] J. F. Gunion, H. E. Haber, Higgs bosons in supersymmetric models (ii). implications for phenomenology, Nuclear Physics B, Particle Physics 278 (3) (1986) 449-492. doi:10. $1016 / 0550-3213(86) 90050-7$.

[74] J. F. Gunion, H. E. Haber, Higgs bosons in supersymmetric models (iii). decays into neutralinos and charginos, Nuclear Physics B, Particle Physics 307 (3) (1988) 445-475. doi : 10.1016/ 0550-3213(88)90259-3. 
[75] J. F. Gunion, H. E. Haber, Errata for higgs bosons in supersymmetric models: I, ii and iii, arXiv:hep-ph/9301205v1 (1993).

[76] T. T. Wu, C. N. Yang, Concept of nonintegrable phase factors and global formulation of gauge fields, Physical Review D 12 (12) (1975) 3845-3857. doi : 10.1103/PhysRevD . 12 . 3845.

[77] S. S. Chern, W. H. Chen, K. S. Lam, Lecture on Differential Geometry, World Scientific Publishing Co. Pte. Ltd., 1999.

[78] O. Nikodym, Sur une generalisation des integrales de mj radon, Fundamenta Mathematicae 15 (1) (1930) 131-179.

[79] R. P. Feynman, Space-time approach to non-relativistic quantum mechanics, Reviews of Modern Physics 20 (2) (1948) 367. doi : 10.1103/RevModPhys . 20.367. 